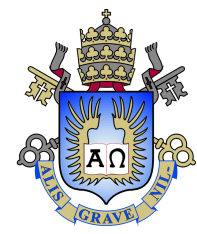

Emilio Rodríguez Hernández

\title{
Orthogonal Multiple Access Schemes in Linear and Non-linear Channels
}

Dissertação de Mestrado

Dissertation presented to the Programa de Pós-graduação em Engenharia Elétrica da PUC-Rio in partial fulfillment of the requirements for the degree of Mestre em Engenharia Elétrica.

Advisor: Prof. Raimundo Sampaio Neto 


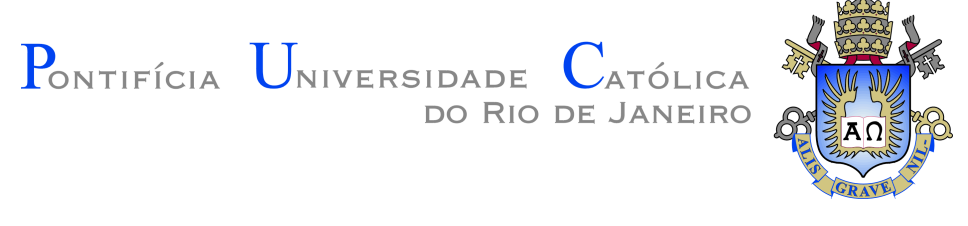

\section{Emilio Rodríguez Hernández}

\section{Orthogonal Multiple Access Schemes in Linear and Non-linear Channels}

Dissertation presented to the Programa de Pós-graduação em Engenharia Elétrica da PUC-Rio in partial fulfillment of the requirements for the degree of Mestre em Engenharia Elétrica. Approved by the Examination Committee.

Prof. Raimundo Sampaio Neto

Advisor

Centro de Estudos em Telecomunições - PUC-Rio

Prof. Marco Antônio Grivet Mattoso Maia

Centro de Estudos em Telecomunicações - PUC-Rio

Dr. Rodrigo Pereira David

Instituto Nacional de Metrologia, Qualidade e Tecnologia -

Inmetro

Dr. Fabian David Backx

Instituto de Pesquisas da Marinha - IPqM

Rio de Janeiro, April the 21st, 2021 
All rights reserved.

\section{Emilio Rodríguez Hernández}

Received the B.S. degree in Telecommunications and Electronic Engineering from the Technology University of Havana "Jose Antonio Echeverria", Havana, Cuba, in 2017.

Bibliographic data

Rodríguez Hernández, Emilio

Orthogonal Multiple Access Schemes in Linear and Nonlinear Channels / Emilio Rodríguez Hernández; advisor: Raimundo Sampaio Neto. - Rio de janeiro: PUC-Rio, Departamento de Engenharia Elétrica, 2021.

v., 83 f: il. color. ; $30 \mathrm{~cm}$

Dissertação (mestrado) - Pontifícia Universidade Católica do Rio de Janeiro, Departamento de Engenharia Elétrica.

Inclui bibliografia

1. Engenharia Elétrica - Teses. 2. Transmissão em blocos; 3. Técnicas de Acesso Múltiplo;. 4. OFDM;. 5. CDMA;. 6. Análise Espectral. I. Sampaio Neto, Raimundo. II. Pontifícia Universidade Católica do Rio de Janeiro. Departamento de Engenharia Elétrica. III. Título. 


\section{Acknowledgments}

First of all, I want to thank to my parents, sister and brother to give me an infinity love and support in all my decisions. They are the motor that impulse my dreams and allows me to reach my goals.

I want to give a special thanks to my dedicated wife, Rocío, for her immense love, understanding and support, without her I would not be the person I am today. Thank you for always trusting me.

I would like to thank my advisor, Prof. Raimundo, for all the time he devoted to me, answering my countless questions. His advice was invaluable for this dissertation and my life. I am very proud that he shared his knowledge, his patience and his friendship with me.

I want to thank to all my colleagues and friends for receiving me and supporting me since the first day in this country, in special way to David, Adila, Randy, Dayrene, Adrian, Ernesto, Julio, Erico, Alireza, Juan Pablo and Jose.

I want to thank to CETUC and all its professors and workers for giving me a warm welcome and make me feel at home.

I would like to thank to PUC-Rio for the exemption of school fees offered by the Programa de Pós-Graduação em Engenharia Elétrica.

Finally, I want to thank the government of Brazil and CNPq for financial support to complete my master's degree. This study was financed in part by the Coordenação de Aperfeiçoamento de Pessoal de Nível Superior - Brasil (CAPES) - Finance Code 001. 


\section{Abstract}

Rodríguez Hernández, Emilio; Sampaio Neto, Raimundo (Advisor). Orthogonal Multiple Access Schemes in Linear and Nonlinear Channels. Rio de Janeiro, 2021. 83p. Dissertação de mestrado - Departamento de Engenharia Elétrica, Pontifícia Universidade Católica do Rio de Janeiro.

Wireless communications are one of the pillars of the development of the new generations of mobile communications. Each generation has used some multiple access technique to take advantage of the channel's resources. This dissertation presents an analysis of two orthogonal multiplexing techniques. Both techniques implement block transmission, where one is combined with the Code Division Multiple Access (CDMA) approach, while the other uses the multicarrier transmission technique Orthogonal Frequency Division Multiplexing (OFDM). The performance and spectral occupation of both techniques and their advantages are analyzed. Analytical expressions for the Power Spectral Density of the signals were obtained, which allowed establishing comparisons between both methods. The study of these multiplexing techniques is carried out in different propagation channels to evaluate the behavior of both systems in general. The three types of channels evaluated in this work are linear and time-invariant, linear and time-variant and, finally, non-linear and time-invariant. Each type of channel was modeled in matrix form for both systems independently. The simulations consider the Zero Forcing and Minimum Mean Squared Error equalizers, assuming a known channel.

\section{Keywords}

Block transmission; $\quad$ Multiple Access Techniques; OFDM; CDMA; Spectral Analysis 


\section{Resumo}

Rodríguez Hernández, Emilio; Sampaio Neto, Raimundo. Esquemas Ortogonais de Acceso Múltiplo em Canais Lineares e Não Lineares. Rio de Janeiro, 2021. 83p. Dissertação de Mestrado - Departamento de Engenharia Elétrica, Pontifícia Universidade Católica do Rio de Janeiro.

As comunicações sem fio são um dos pilares do desenvolvimento das novas gerações de comunicações móveis. Cada geração tem usado alguma técnica de acesso múltiplo para aproveitar os recursos do canal. Esta dissertação apresenta uma análise de duas técnicas de multiplexação ortogonal. Ambas técnicas implementam a transmissão em blocos, onde uma delas se combina com a abordagem Code Division Multiple Access (CDMA), enquanto a outra usa a técnica de transmissão multiportadora Orthogonal Frequency Division Multiplexing (OFDM). O desempenho e a ocupação espectral de ambas técnicas e as suas vantagens são analisados neste trabalho. Expressões analíticas para a Densidade Espectral de Potência dos sinais foram obtidas e permitiram estabelecer comparações entre os dois métodos. O estudo dessas técnicas de multiplexação é realizado em diferentes canais de propagação para avaliar o comportamento de ambos sistemas de uma forma geral. Os três tipos de canais avaliados neste trabalho são: linear e invariante no tempo, linear e variante no tempo e, finalmente, não linear e invariante no tempo. Cada tipo de canal foi modelado em forma matricial para ambos sistemas de forma independente. As simulações consideram os equalizadores Zero Forcing e Minimum Mean Square Error, assumindo um canal conhecido.

\section{Palavras-chave}

Transmissão em blocos; Técnicas de Acesso Múltiplo; OFDM; CDMA; Análise Espectral 


\section{Table of contents}

1 Introduction $\quad 15$

$\begin{array}{lll}1.1 & \text { Document Organization } & 17\end{array}$

$\begin{array}{ll}1.2 \text { Adopted Notation } & 17\end{array}$

2 Block Transmission Schemes in time-invariant linear channels $\quad 19$

2.1 Multicarrier Block Transmission - OFDMA 19

2.1.1 OFDMA matrix model of transmission and reception 20

2.1.1.1 Uplink configuration $\quad 23$

$\begin{array}{ll}\text { 2.1.1.2 Downlink configuration } & 24\end{array}$

2.1.2 OFDMA Equalization Strategies 25

2.2 Single Carrier Block Transmission - CS-CDMA 26

2.2.1 CS-CDMA matrix model of transmission and reception 26

2.2.2 CS-CDMA Equalization Strategies 28

2.3 Numerical Results: OFDMA and CS-CDMA in time-invariant linear channels $\quad 29$

2.4 Spectral Analysis 34

2.4.1 Systems without guard interval 36

2.4.1.1 OFDMA 36

2.4.1.2 CS-CDMA 37

2.4.1.3 Numerical results of OFDMA and CS-CDMA without guard interval 38

2.4.2 Systems with guard interval 40

$\begin{array}{lll}2.4 .2 .1 & \text { CP-OFDMA } & 40\end{array}$

2.4.2.2 ZP-CS-CDMA 41

2.4.2.3 Numerical results of OFDMA and CS-CDMA with guard interval 43

3 Block Transmission Schemes in time-varying linear channels 46

3.1 OFDMA in time-varying linear environments 46

3.1.1 OFDMA with Zero Forcing Equalization 48

3.1.2 OFDMA with Minimum Mean Square Error Equalization 48

3.2 CS-CDMA in time-varying linear environments 49

3.2.1 CS-CDMA with Zero Forcing Equalization 50

3.2.2 CS-CDMA with Minimum Mean Square Error Equalization 51

3.3 Numerical Results: OFDMA and CS-CDMA in time-varying linear channels $\quad 52$

4 Block Transmission Schemes in time-invariant non-linear channels $\quad 59$

4.1 OFDMA in non-linear channels $\quad 59$

4.1.1 OFDMA Equalization Strategies 63

4.2 CS-CDMA in non-linear channels 64

4.2.1 CS-CDMA Equalization Strategies 66

4.3 Numerical Results: OFDMA and CS-CDMA in time-invariant nonlinear channels $\quad 66$

$\begin{array}{lll}5 & \text { Conclusions } & 73\end{array}$ 
Bibliography

A PSD of Block Transmission Systems

78

B Analytical Expression of BER for OFDM Systems 


\section{List of figures}

Figure 2.1 Discrete transmission model of CP-OFDM [1]. 21

Figure 2.2 Discrete model for the reception of CP-OFDM [1]. 21

Figure 2.3 Sequence of $B$ transmitted blocks by $k$-th user in CSCDMA [2].

Figure 2.4 BER vs $E_{B} / N_{0}$ curves to CS-CDMA systems with guard interval $\mathrm{ZP}$ and $\mathrm{CP}(L=4), N=8, K=8, B=8$ and $P=4$, in uplink configuration.

Figure 2.5 BER vs $E_{B} / N_{0}$ curves to CS-CDMA systems with guard interval $\mathrm{ZP}$ and $\mathrm{CP}(L=4), N=12, K=4, B=12$ and $P=4$, in uplink configuration.

Figure 2.6 BER vs $E_{B} / N_{0}$ curves to CS-CDMA systems with guard interval $\mathrm{ZP}$ and $\mathrm{CP}(L=4), N=8, K=8, B=8$ and $P=4$, in downlink configuration.

Figure 2.7 BER vs $E_{B} / N_{0}$ curves to CS-CDMA systems with guard interval $\mathrm{ZP}$ and $\mathrm{CP}(L=4), N=12, K=4, B=12$ and $P=4$, in downlink configuration.

Figure 2.8 BER vs $E_{B} / N_{0}$ curves of OFDMA and CS-CDMA systems with a set of parameters $L=4, M=64, N=8$, $K=8, B=8$ and $P=4$, in uplink configuration.

Figure 2.9 BER vs $E_{B} / N_{0}$ curves of OFDMA and CS-CDMA systems with a set of parameters $L=4, M=48, N=12$, $K=4, B=12$ and $P=4$, in uplink configuration.

Figure $2.10 \mathrm{BER}$ vs $E_{B} / N_{0}$ curves of OFDMA and CS-CDMA systems with a set of parameters $L=4, M=64, N=8$, $K=8, B=8$ and $P=4$, in downlink configuration.

Figure 2.11 BER vs $E_{B} / N_{0}$ curves of OFDMA and CS-CDMA systems with a set of parameters $L=4, M=48, N=12$, $K=4, B=12$ and $P=4$, in downlink configuration.

Figure 2.12 Transmission matrices multiplication $\boldsymbol{\Psi} \boldsymbol{\Psi}^{H}$ of OFDMA with $M=64$ and without guard interval.

Figure 2.13 Transmission matrices multiplication $\Psi \Psi^{H}$ of CSCDMA with $N=8, K=8, B=8$ and without guard interval.

Figure 2.14 Power Spectral Density of OFDMA system with FFT $64(M=64)$ and CS-CDMA system with $N=8, K=8$ and $B=8$, both without guard interval normalized by the maximum point $\left(R_{D}\right.$ is the data symbol rate).

Figure 2.15 Power Spectral Density of OFDMA system with FFT $64(M=64)$ and CS-CDMA system with $N=8, K=8$ and $B=8$, both without guard interval normalized by area.

Figure 2.16 Percentage out of band: OFDMA system with FFT 64 $(M=64)$ and CS-CDMA system with $N=8, K=8$ and $B=8.41$ Figure 2.17 Transmission matrices multiplication $\boldsymbol{\Psi} \boldsymbol{\Psi}^{H}$ of $\mathrm{CP}$ OFDMA with $M=64$ and $L=4$. 
Figure 2.18 Transmission matrices multiplication $\boldsymbol{\Psi} \Psi^{H}$ of ZP-CSCDMA with $N=8, K=8, B=8$.

Figure 2.19 Power Spectral Density of CP-OFDMA system with FFT $64(M=64)$ and ZP-CS-CDMA system with $N=8, K=8$ and $B=8$, both with a guard interval of $L=4$ and normalized by the maximum point.

Figure 2.20 Power Spectral Density of CP-OFDMA system with FFT $64(M=64)$ and ZP-CS-CDMA system with $N=8, K=8$ and $T=8$, both with a guard interval of $L=4$ and normalized by the area.

Figure 2.21 Percentage out of band to CP-OFDMA system with FFT $64(M=64)$ and ZP-CS-CDMA system with $N=8, K=8$ and $B=8$, and both with $L=4$.

Figure 3.1 BER vs $E_{B} / N_{0}$ curves of OFDMA and CS-CDMA systems with a set of parameters $f_{d} T_{s}=10^{-6}, L=4, M=64$, $N=8, K=8, B=8$ and $P=4$, in uplink configuration.

Figure 3.2 BER vs $E_{B} / N_{0}$ curves of OFDMA and CS-CDMA systems with a set of parameters $f_{d} T_{s}=10^{-4}, L=4, M=64$, $N=8, K=8, B=8$ and $P=4$, in uplink configuration.

Figure 3.3 BER vs $E_{B} / N_{0}$ curves of OFDMA and CS-CDMA systems with a set of parameters $f_{d} T_{s}=10^{-3}, L=4, M=64$, $N=8, K=8, B=8$ and $P=4$, in uplink configuration.

Figure 3.4 BER vs $E_{B} / N_{0}$ curves of OFDMA and CS-CDMA systems with a set of parameters $f_{d} T_{s}=10^{-6}, L=4, M=64$, $N=8, K=8, B=8$ and $P=4$, in downlink configuration.

Figure 3.5 BER vs $E_{B} / N_{0}$ curves of OFDMA and CS-CDMA systems with a set of parameters $f_{d} T_{s}=10^{-4}, L=4, M=64$, $N=8, K=8, B=8$ and $P=4$, in downlink configuration.

Figure 3.6 BER vs $E_{B} / N_{0}$ curves of OFDMA and CS-CDMA systems with a set of parameters $f_{d} T_{s}=10^{-3}, L=4, M=64$, $N=8, K=8, B=8$ and $P=4$, in downlink configuration.

Figure 3.7 BER vs $E_{B} / N_{0}$ curves of OFDMA and CS-CDMA systems with a set of parameters $f_{d} T_{s}=10^{-6}, L=4, M=48$, $N=12, K=4, B=12$ and $P=4$, in uplink configuration.

Figure 3.8 BER vs $E_{B} / N_{0}$ curves of OFDMA and CS-CDMA systems with a set of parameters $f_{d} T_{s}=10^{-4}, L=4, M=48$, $N=12, K=4, B=12$ and $P=4$, in uplink configuration.

Figure 3.9 BER vs $E_{B} / N_{0}$ curves of OFDMA and CS-CDMA systems with a set of parameters $f_{d} T_{s}=10^{-3}, L=4, M=48$, $N=12, K=4, B=12$ and $P=4$, in uplink configuration.

Figure 4.1 Non-linear transformation scheme.

Figure 4.2 Graphic representation of $\left|w_{i}\right|=f\left(\left|z_{i}\right|\right)$.

Figure 4.3 BER vs $E_{\text {sat }}^{b} / N_{0}$ curves to OFDMA non-linear systems without considering the OBO with $L=4, N=8, K=8, B=8$ and $P=4$, in downlink configuration and using the $\mathrm{ZF}$ equalizer. 68 
Figure 4.4 BER vs $E_{\text {sat }}^{b} / N_{0}$ curves to OFDMA non-linear systems without considering the $\mathrm{OBO}$ with $L=4, N=8, K=8, B=8$ and $P=4$, in downlink configuration and using the MMSE equalizer.

Figure 4.5 BER vs $E_{\text {sat }}^{b} / N_{0}$ curves to CS-CDMA non-linear systems without considering the OBO with $L=4, N=8, K=8, B=8$ and $P=4$, in downlink configuration and using the $\mathrm{ZF}$ equalizer. 69

Figure 4.6 BER vs $E_{\text {sat }}^{b} / N_{0}$ curves to CS-CDMA non-linear systems without consider the OBO with $L=4, N=8, K=8, B=8$ and $P=4$, in downlink configuration and using the MMSE equalizer. 70

Figure 4.7 BER vs $E_{\text {sat }}^{b} / N_{0}$ curves to OFDMA non-linear systems considering the OBO with $L=4, N=8, K=8, B=8$ and $P=4$, in downlink configuration and using the ZF equalizer.

Figure 4.8 BER vs $E_{\text {sat }}^{b} / N_{0}$ curves to OFDMA non-linear systems considering the OBO with $L=4, N=8, K=8, B=8$ and $P=4$, in downlink configuration and using the MMSE equalizer. 71

Figure 4.9 BER vs $E_{\text {sat }}^{b} / N_{0}$ curves to CS-CDMA non-linear systems considering the OBO with $L=4, N=8, K=8, B=8$ and $P=4$, in downlink configuration and using the $\mathrm{ZF}$ equalizer.

Figure 4.10 BER vs $E_{\text {sat }}^{b} / N_{0}$ curves to CS-CDMA non-linear systems considering the OBO with $L=4, N=8, K=8, B=8$ and $P=4$, in downlink configuration and using the MMSE equalizer. 72

Figure B.1 BER vs $E_{b} / N_{0}$ curves of OFDM computed analytically and through simulation with $M=64$ and $48, L=4$ and $P=4 . \quad 83$

Figure B.2 BER vs $E_{b} / N_{0}$ curves of OFDM computed analytically and through simulation with $M=64$ and $48, L=8$ and $P=8$. 


\section{List of tables}

Table 2.1 Simulation parameters - random, multipath, linear and time-invariant channel.

Table 3.1 Simulation parameters - random, multipath, linear and time-varying channel. 


\section{List of Abreviations}

$4 \mathrm{G}$ - Fourth Generation

$5 \mathrm{G}$ - Fifth Generation

AWGN - Additive White Gaussian Noise

BER - Bit Error Rate

CDMA - Code Division Multiple Access

$\mathrm{CP}$ - Cyclic Prefix

CP-OFDM - Cyclic Prefix Orthogonal Frequency Division Multiplexing

CP-OFDMA - Cyclic Prefix Orthogonal Frequency Division Multiple Access

CS-CDMA - Chip Spread Code Division Multiple Access

DFT - Discrete Fourier Transform

DS-CDMA - Direct Sequence Code Division Multiple Access

FFT - Fast Fourier Transform

FIR - Finite Impulse Response

IBI - Inter-Block Interference

IBO - Input Back-off

IDFT - Inverse Discrete Fourier Transform

IoT - Internet of Things

ISI - Inter-Symbol Interference

MAI - Multiple Access Interference

MMSE - Minimum Mean Square Error

OBO - Output Back-off

OFDM - Orthogonal Frequency Division Multiplexing

OFDMA - Orthogonal Frequency Division Multiple Access

PA - Power Amplifier

PAPR - Peak-to-Average Power Ratio

QoE - Quality of experience

QPSK - Quadrature Phase Shift Keying

RBS - Radio Base Station

SNR - Signal-to-noise ratio

ZF - Zero Forcing

ZP - Zero Padding

ZP-CS-CDMA - Zero Padding Chip Spread Code Division Multiple Access 
While working on completing your goal, you may encounter possible setbacks. Instead of beating yourself up, look at them as opportunities to learn and grow.

Kyle Nussen, Time Management. 


\section{Introduction}

Wireless communications occupy an increasingly important space in our society. In the last decade, the advances of communication systems have been accelerated for many reasons such as the exponential growth of the number of users and the need for lower latency and higher data rates. With the emergence of technologies such as smartphones and Internet of Things (IoT), the energy efficiency becomes an important aspect to improve in systems.

The inter-connection of billions of smart devices was made possible with the development of the IoT [3]. This technology and the growing demand of the streaming services introduce significant modifications in mobile systems to guarantee high speeds and high spectral efficiency [4]. The expected growth in the quality of data exchange in the coming decades brings with it the need to create and implement the next generation of mobile communications.

The next generation has to coexist with different technologies, substantially increasing the transmission capacity. These demands must be met by innovative techniques at different network layers, without the cost of bandwidth and power consumption [5]. The fifth generation (5G) mobile network is growing and should provide an improvement over the current $4 \mathrm{G}$ in capacity and several functionalities to fulfill the rising user expectation of quality of experience (QoE). The huge and never ending growth in volume of data traffic is one of the main drivers of $5 \mathrm{G}$ [6]. The main challenge is to develop wireless communication technologies that will support the volume of data traffic in the coming years.

Communication systems are affected by multipath channels, such that mobile systems can suffer significant performance limitations. The level of interference is a worrying effect for conventional systems that implement sequential transmission. An alternative to address this problem is the adoption of block transmission systems. These systems are affected by the inter-block interference (IBI), which can be avoided by the insertion of a guard interval [7]. The insertion of an appropriate guard interval also allows a significant simplification of the channel estimation and equalization process [2]. After insertion of the guard interval, the resulting symbol block can be transmitted using a multicarrier or single-carrier scheme. 
Each new generation of mobile systems seeks to improve on the previous generation. Features such us data transmission capacity, efficiency, portability and others are aspects to consider when updating previous mobile generations. With each transition the requirements become more demanding, prompting the scientific community to find new alternatives to create innovative solutions. In order to increase the communication capacity of mobile systems to support a large number of users, new ways of taking advantage of the channel's resources are being investigated. Simultaneous data transmission over the same channel is a viable way to take advantage of spectrum resources. Multiple access schemes allow the implementation of simultaneous data transmission.

Each generation of mobile communications implements some multiplexing technique based on time, frequency or code division, even combinations between them [8]. Multiple access schemes are divided into two categories: orthogonal and non-orthogonal. Orthogonal schemes use orthogonal resources such as disjoint time windows reserved for different users, different frequency carriers, or orthogonal codes, allowing users to be separated at the reception. Orthogonal schemes are widely used even by the current $4 \mathrm{G}$ generation.

In the literature, several related works combine different multiple access techniques. In this direction, Direct Sequence-Code Division Multiple Access (DS-CDMA) and Orthogonal Frequency Division Multiplexing (OFDM) are combined to obtain new multiple access schemes. Multicarrier techniques such as Multicarrier CDMA [9] and Multicarrier DS-CDMA [10, 11] were proposed with the aim of improving performance. A major drawback that affects multicarrier systems is the high Peak-to-Average Power Ratio (PAPR). This reason motivated the study of single-carrier approaches for block transmission systems $[12,13]$.

This dissertation focuses on the study of two multiple access techniques proposed for using in block transmission systems. One combines single carrier block transmission using the CDMA multiplexing technique, known as Chip Spread Code Division Multiple Access (CS-CDMA) [2]. The other multiple access technique discussed is known as Orthogonal Frequency Division Multiple Access (OFDMA) [14]. In this work, the expressions for the power spectral density are obtained and the characteristics of out-of-band emission of these systems are analyzed. The scenarios analyzed here were studied in depth in order to investigate the behavior of the two block transmission systems in multipath channels with marked differences. Three kinds of channels were evaluated, the time-invariant linear channel, the time-varying linear channel and, finally, the time-invariant non-linear channel. 


\section{1 \\ Document Organization}

The chapters of this dissertation are organized as follows: in Chapter 2, the OFDMA and CS-CDMA systems in time-invariant linear channels are analyzed, starting with the explanation of the multicarrier transmission technique OFDM with cyclic prefix. Then, the OFDMA and CS-CDMA systems are characterized through their matrix models and the detection process using two well-known detectors: Zero Forcing (ZF) and Minimum Mean Square Error (MMSE). The CS-CDMA system in this chapter is analyzed using the Cyclic Prefix (CP) and Zero Padding (ZP) guard intervals. Both techniques are studied in uplink and downlink configuration. The numerical results are presented in time-invariant linear channels, where the performance of each multiple access technique is considered. A spectral analysis of both systems is performed and the expressions to compute the power spectral density and the out-of-band emission of these systems are analyzed.

In Chapter 3, the transmission is carried out through time-varying linear channels, where channels with slow and fast variations are analyzed. The type of variation is determined by the product $f_{d} T_{s}$, where $f_{d}$ is the Doppler frequency and $T_{s}$ is the symbol block duration. The OFDMA and CS-CDMA matrix models are studied and the $\mathrm{CP}$ and $\mathrm{ZP}$ guard intervals are used respectively. The ZF and MMSE equalizer techniques are used in the detection process by both techniques. The numerical results of the systems in timevarying linear channels are shown through the performance curves.

In Chapter 4, time-invariant non-linear channels are discussed. In this part, the model used to treat the non-linear channels is explained for both systems. The ZF and MMSE equalizers are used again and the performance curves are analyzed to obtain the behavior of both systems in this kind of channels.

Finally, Chapter 5 is dedicated to presenting the final conclusions of this study. We summarize the main results and suggest some directions for further researches.

\section{2}

\section{Adopted Notation}

Throughout this work, we have employed the following notation: lowercase and uppercase bold letters represent vectors (e.g. a) and matrices (e.g. B) respectively. $\mathbf{I}_{N}$ represents a square identity matrix $N \times N$. 0 denotes a matrix or vector with all its elements equal to zero. The operators 
$(.)^{T},(.)^{H},(.)^{-1}$ and ()$^{\dagger}$ represent the transpose, Hermitian, inverse and pseudoinverse of a matrix respectively. The operator $\mathbb{E}[$.$] denotes the expected value.$ 


\section{Block Transmission Schemes in time-invariant linear channels}

In this chapter, two types of systems that implement a block transmission scheme are presented. Both approaches are classified as orthogonal multiplexing techniques, where one of them is multicarrier and another is single-carrier. The multicarrier system is based in the Orthogonal Frequency Division Multiplexing (OFDM) parallel transmission method while the single-carrier approach uses Code Division Multiple Access (CDMA) technique. The mathematical discrete models for both approaches are covered in this chapter.

\section{1}

\section{Multicarrier Block Transmission - OFDMA}

The combination of spread spectrum and frequency hopping with multicarrier transmission originated a multiple access hybrid alternative: Orthogonal Frequency Division Multiple Access (OFDMA) [8]. OFDMA is based on the multicarrier transmission technique OFDM. Therefore, the first necessary step to understand OFDMA method is analyzing how the OFDM works.

OFDM is a parallel transmission technique that arises to mitigate the serial transmission systems problems, which uses single-carrier modulation. A significant advantage over the serial transmission is the reduction of channel delay spreading and therefore, the Inter-Symbol Interference (ISI). By dividing an input signal with transmission rate $R$, in $M$ sub-carriers with lower transmission rate $(R / M)$, each sub-channel will have a fading approximately flat in frequency. While the original input signal in wide band suffers a severe selective fading effect induced by the radio mobile channel [15].

OFDM consists in the parallel data transmission using orthogonal subcarriers. Each sub-carrier transmits a sequence of digitally modulated pulses. The OFDM technique is an evolution of Frequency Division Multiplexing (FDM), which uses guard bands to separate the corresponding signal subcarriers spectra. FDM with its implementation entails an inadmissible bandwith waste for current transmission systems.

This problem is avoided by OFDM through the spectral superposition of $M$ sub-carriers with frequencies spaced in $1 / T_{s}$, where $T_{s}$ represents an interval 
of OFDM sub-symbol duration. Each OFDM symbol is formed by a set of $M$ sub-symbols belonging to the employed modulation scheme [1].

Despite the existence of spectral superposition, the spectra of each subcarrier are mathematically orthogonal between them. The spacing among subcarriers is determined by allocating each sub-carrier in the position corresponding to the spectral nulls of the remaining sub-carriers. In this sense, the orthogonality condition in frequency domain is maintained through the use of rectangular transmission pulses. Then, the Fourier Transform corresponding to these rectangular pulses is a sinc $(f)$ centered in the frequency associated to each sub-channel. In this way, there will be no overlap at the central frequency, where the information is contained [1].

OFDMA involves the attribution of one or several frequency sub-bands to a given user with the limitation that the spacing among sub-bands is equal to $1 / T_{s}$, the same relationship used in OFDM to maintain the orthogonality between sub-carriers [14].

\subsection{1}

\section{OFDMA matrix model of transmission and reception}

The OFDMA matrix model is based on the discrete OFDM model for signals transmission in time-invariant linear channels [7]. This description takes into account the addition of Cyclic Prefix (CP) as guard interval to reduce the Inter-Block Interference (IBI). CP was selected in this work since the $4 \mathrm{G}$ and other well-known protocols use this configuration. This kind of interference is usually analyzed in OFDM systems. The guard interval must fulfill that the convolution of the transmitted signal and the impulse response of the propagation channel does not interfere at reception of the next symbol [16].

$\mathrm{CP}$ is a cyclic extension that corresponds to the insertion of a copy of the last $L$ samples of the OFDM symbol in the beginning of each symbol. Once the signal is received, the samples contained in the guard interval are discarded. This operation allows to discard the redundant information, retrieving the initial dimension $M[16]$.

Before describing the OFDMA matrix model, we present the transmission process of CP-OFDM signals. These concepts are used to build our matrix representation for OFDMA systems.

The normalized matrix of Discrete Fourier Transform (DFT) is represented by $\mathbf{W}_{M}$ such as $\mathbf{W}_{M}^{H} \mathbf{W}_{M}=\mathbf{W}_{M} \mathbf{W}_{M}^{H}=\mathbf{I}_{M}$. Its components are given by

$$
\left[\mathbf{W}_{M}\right]_{m, v}=\frac{1}{\sqrt{M}} e^{-j \frac{2 \pi}{M} m v} \quad 0 \leq m, v \leq M-1 .
$$

The transmission signal CP-OFDM at instant $i, \mathbf{s}_{c p}(i)$, is obtained by 
adding a guard interval to the original transmission signal $\mathbf{s}(i)=\mathbf{W}_{M}^{H} \mathbf{d}(i)$. As mentioned above this guard interval is formed by the last $L$ samples of the signal $\mathbf{s}(i)$. Thus, the number of transmitted samples is $G=M+L$. Figure 2.1 illustrates the discrete process generation of a symbol CP-OFDM, where it was supposed $i=0$ and this index is temporally suppressed for convenience of notation.

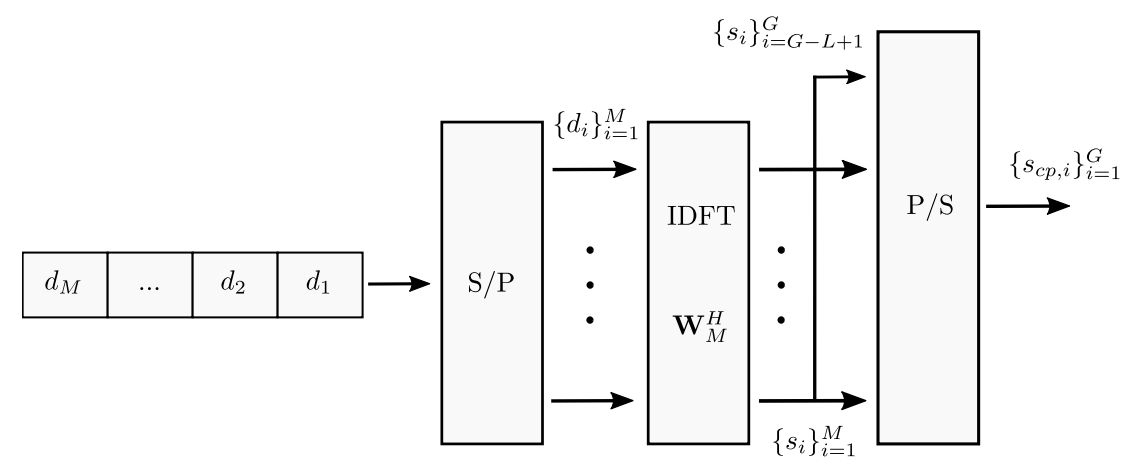

Figure 2.1: Discrete transmission model of CP-OFDM [1].

At instant $i$, the transmission vector $\mathbf{s}_{c p}(i)$ has a dimension $G \times 1$ and it is defined as

$$
\mathbf{s}_{c p}(i)=\mathbf{M}_{c p} \mathbf{d}(i)
$$

where

$$
\mathbf{M}_{c p}=\left[\begin{array}{c}
\mathbf{W}_{c p}^{H} \\
\mathbf{W}_{M}^{H}
\end{array}\right]
$$

and $\mathbf{W}_{c p}^{H}$ is a matrix composed by the last $L$ rows of the Inverse Discrete Fourier Transform (IDFT) matrix $\mathbf{W}_{M}^{H}$.

The transmitted signal is propagated through a channel modeled by a discrete filter with impulse response given by $\mathbf{h}_{P}=\left[\begin{array}{llll}h_{0} & h_{1} & \ldots & h_{P-1}\end{array}\right]^{T}$ with dimension $P$. The received CP-OFDM signal is also affected by the additive white Gaussian noise (AWGN), which is modeled by $\mathbf{n}_{c p}(i)$. Figure 2.2 shows the structure of a CP-OFDM discrete receiver.

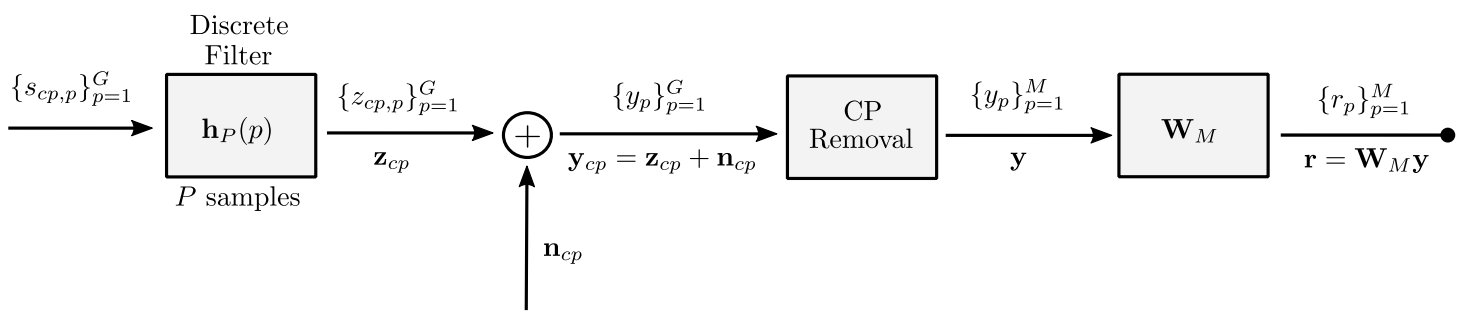

Figure 2.2: Discrete model for the reception of CP-OFDM [1].

An important aspect in a real system is to satisfy the relationship $M>L \geq P-1$. The continuous transmission of CP-OFDM symbols and the 
channel action induce a superposition between the received OFDM symbols. Then, the $i$-th observation with dimension $G \times 1$ can be expressed by [7]

$$
\mathbf{y}_{c p}(i)=\mathbf{H M}_{c p} \mathbf{d}(i)+\mathbf{H}_{i b i} \mathbf{M}_{c p} \mathbf{d}(i-1)+\mathbf{n}_{c p}(i),
$$

where the matrix $\mathbf{H}$ is a $G \times G$ Toeplitz matrix that represents the channel behavior. Its first column is given by $\left[\begin{array}{lllllll}h_{0} & h_{1} & \ldots & h_{P-1} & 0_{1} & \ldots & 0_{G-P}\end{array}\right]^{T} \cdot \mathbf{H}_{i b i}$ is an $G \times G$ upper triangular matrix that represents the common IBI effect in OFDM systems. After CP removal, the first $L$ rows of all matrices in (2-4) are deleted, which allows the suppression of IBI. Taking into account the condition $L \geq P-1$, the $i$-th observation of dimension $M \times 1$ can be expressed as

$$
\mathbf{y}(i)=\left[\mathbf{0}_{M \times G} \mathbf{I}_{M}\right] \mathbf{y}_{c p}(i)=\operatorname{Circ}(\mathbf{h}) \mathbf{W}_{M}^{H} \mathbf{d}(i)+\mathbf{n}(i),
$$

where the vector $\mathbf{n}(i)$ with length $M \times 1$ represents the noise vector with null mean and covariance matrix

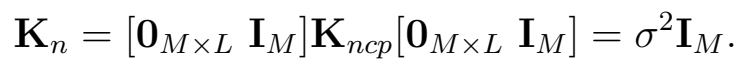

The addition and removal of CP, through pre-defined matrices do not modify the noise vector statistics. In (2-5), $\mathbf{C i r c}(\mathbf{h})$ is a circulant matrix with dimension $M \times M$ given by

$$
\operatorname{Circ}(\mathbf{h})=\left[\begin{array}{ccccccc}
h_{0} & 0 & & 0 & h_{P-1} & \ldots & h_{1} \\
\vdots & \ddots & \ddots & & \ddots & \ddots & \vdots \\
h_{P-2} & & \ddots & \ddots & & \ddots & h_{P-1} \\
h_{P-1} & \ddots & & \ddots & \ddots & & 0 \\
0 & \ddots & \ddots & & \ddots & \ddots & \vdots \\
\vdots & \ddots & \ddots & \ddots & & \ddots & 0 \\
0 & \ldots & 0 & h_{P-1} & h_{P-2} & \ldots & h_{0}
\end{array}\right],
$$

where the first column is $\mathbf{h}=\left[\begin{array}{llllll}h_{0} & \ldots & h_{P-1} & 0_{1} & \ldots & 0_{M-P}\end{array}\right]^{T}$.

The use of guard interval as cyclic extension allows the linear convolution operation between the channel impulse response and the CP-OFDM signal transmission to be treated as a circular convolution [7].

After demodulation process with the DFT matrix represented by $\mathbf{W}_{M}$ the received signal is expressed by

$$
\mathbf{r}(i)=\mathbf{W}_{M} \operatorname{Circ}(\mathbf{h}) \mathbf{W}_{M}^{H} \mathbf{d}(i)+\mathbf{W}_{M} \mathbf{n}(i) .
$$

The pre and pos multiplication of the circulant matrix (2-7) by the DFT and IDFT matrices result in a diagonal matrix. This is supported by the fact that any circulant matrix is diagonalizable through the Discrete Fourier Transform properties. Therefore, (2-8) can be rewritten as 


$$
\mathbf{r}(i)=\operatorname{Diag}(\mathbf{q}) \mathbf{d}(i)+\mathbf{n}^{\prime}(i)
$$

The components of the resultant diagonal matrix $\operatorname{Diag}(\mathbf{q})$ are formed by the components of the discrete frequency response of the channel [7]

$$
\mathbf{q}=\sqrt{M} \mathbf{W}_{M} \mathbf{h}
$$

It is important to note that the statistics of the Gaussian vector do not change with the application of an orthogonal transformation as done by $\mathbf{W}_{M}$. Therefore, the vector $\mathbf{n}^{\prime}(i)=\mathbf{W}_{M} \mathbf{n}(i)$ is still a complex Gaussian vector with null mean and covariance matrix $\mathbf{K}_{n}^{\prime}=\sigma^{2} \mathbf{I}_{M}$. In this way, the noise vectors $\mathbf{n}(i)$ in (2-5) and $\mathbf{n}^{\prime}(i)$ in (2-9) are equivalent.

In order to better describe the OFDMA matrix model we separate the description of the uplink and dowlink configurations.

\subsubsection{1}

\section{Uplink configuration}

The reverse link or uplink configuration occurs when users perform the transmission and the Radio Base Station (RBS) carries out the reception. The RBS receives the information sent by all users. A simple case composed by only two active users $(K=2)$ is presented. The users start the transmission, where each one of them transmits through its corresponding channel. After removal of cyclic extension, the channel matrices turn into circulant matrices and the received vector by the RBS is given as

$$
\mathbf{y}=\operatorname{Circ}\left(\mathbf{h}_{1}\right) \mathbf{W}_{M}^{H}\left[\begin{array}{c}
\mathbf{d}_{1} \\
0_{1} \\
\vdots \\
0_{M-J}
\end{array}\right]+\operatorname{Circ}\left(\mathbf{h}_{2}\right) \mathbf{W}_{M}^{H}\left[\begin{array}{c}
0_{1} \\
\vdots \\
0_{M-J} \\
\mathbf{d}_{2}
\end{array}\right]+\mathbf{n}
$$

where

$$
\mathbf{d}_{i}=\left[\begin{array}{c}
d_{i 1} \\
d_{i 2} \\
\vdots \\
d_{i J}
\end{array}\right], \quad i=1,2 \quad J \leq \frac{M}{2},
$$

$\mathbf{h}_{1}$ and $\mathbf{h}_{2}$ are the impulse responses of the discrete low-pass equivalent of the channels corresponding to each user. Considering the pre/pos multiplication by DFT and IDFT matrices, the circulant matrix turns into a diagonal matrix and (2-11) can be rewritten as 


$$
\mathbf{r}=\operatorname{Diag}\left(\mathbf{q}_{1}\right)\left[\begin{array}{c}
\mathbf{d}_{1} \\
0_{1} \\
\vdots \\
0_{M-J}
\end{array}\right]+\operatorname{Diag}\left(\mathbf{q}_{2}\right)\left[\begin{array}{c}
0_{1} \\
\vdots \\
0_{M-J} \\
\mathbf{d}_{2}
\end{array}\right]+\mathbf{n}^{\prime}
$$

where $\mathbf{q}_{1}$ and $\mathbf{q}_{2}$ are transform coefficients of discrete impulse responses of channels, expressed in (2-10). Then, from (2-13) we have that

$$
\mathbf{r}=\operatorname{Diag}(\mathbf{q})\left[\begin{array}{l}
\mathbf{d}_{1} \\
\mathbf{d}_{2}
\end{array}\right]+\mathbf{n}^{\prime},
$$

where

$$
\operatorname{Diag}(\mathbf{q})=\left[\begin{array}{cc}
\operatorname{Diag}\left(\mathbf{q}_{1}^{\prime}\right) & 0 \\
0 & \operatorname{Diag}\left(\mathbf{q}_{2}^{\prime}\right)
\end{array}\right]
$$

In the example, $\mathbf{q}_{1}^{\prime}$ contains the $J$ first coefficients of $\mathbf{q}_{1}$ and $\mathbf{q}_{2}^{\prime}$ contains the last $J$ coefficients of $\mathbf{q}_{2}$. In (2-14) is expressed the uplink configuration, which allows to perform a simplified equalization by mean of a diagonal matrix. This characteristic is an important advantage of OFDMA. Note that (2-14) is pretty similar to (2-9), which helps to check the procedure for obtaining the uplink equation.

\subsubsection{2}

\section{Downlink configuration}

In this communication the RBS transmits the information corresponding to each of the $K$ user. First, the transmission process is carried out at the RBS,

$$
\mathbf{s}=\mathbf{W}_{M}^{H}\left[\begin{array}{c}
\mathbf{d}_{\mathbf{1}} \\
\mathbf{d}_{\mathbf{2}} \\
\vdots \\
\mathbf{d}_{\mathbf{K}}
\end{array}\right]=\mathbf{W}_{M}^{H} \mathbf{d}
$$

The IDFT matrix can be partitioned in $K$ sub-matrices

$$
\mathbf{W}_{M}^{H}=\left[\begin{array}{llllll}
\mathbf{W}_{1}^{H} & \mathbf{W}_{2}^{H} & \ldots & \mathbf{W}_{k}^{H} & \ldots & \mathbf{W}_{K}^{H}
\end{array}\right],
$$

where the number of columns of $\mathbf{W}_{i}^{H}$, with $i=1, \ldots, K$, equal the dimension of the data vector $\mathbf{d}_{i}$ associated to user $i$. Note that in downlink scenario, each user receives the information through its own channel. In the case of user $k$, after removing the $\mathrm{CP}$ the received signal is given by

$$
\mathbf{r}_{k}=\operatorname{Circ}\left(\mathbf{h}_{k}\right) \mathbf{W}_{M}^{H} \mathbf{d}+\mathbf{n},
$$


and expanding the expression (2-18) we have

$$
\begin{array}{r}
\mathbf{r}_{k}=\operatorname{Circ}\left(\mathbf{h}_{k}\right) \mathbf{W}_{1}^{H} \mathbf{d}_{1}+\operatorname{Circ}\left(\mathbf{h}_{k}\right) \mathbf{W}_{2}^{H} \mathbf{d}_{2}+\ldots+ \\
\operatorname{Circ}\left(\mathbf{h}_{k}\right) \mathbf{W}_{k}^{H} \mathbf{d}_{k}+\ldots+\operatorname{Circ}\left(\mathbf{h}_{k}\right) \mathbf{W}_{K}^{H} \mathbf{d}_{K}+\mathbf{n} .
\end{array}
$$

Then, the DFT matrix is composed by

$$
\mathbf{W}_{M}=\left[\begin{array}{c}
\mathbf{W}_{1} \\
\mathbf{W}_{2} \\
\vdots \\
\mathbf{W}_{k} \\
\vdots \\
\mathbf{W}_{K}
\end{array}\right] .
$$

In the reception the DFT operation is applied. In this case, the user $k$ received signal $\mathbf{r}_{k}$ in (2-19) is multiplied by $\mathbf{W}_{k}$ and using the property of the orthogonal matrices, we obtain

$$
\mathbf{W}_{k} \mathbf{r}_{k}=\operatorname{Diag}\left(\mathbf{q}_{k k}\right) \mathbf{d}_{k},
$$

where $\mathbf{q}_{k k}=\sqrt{\mathbf{M}} \mathbf{W}_{k} \mathbf{h}_{k}$, which is defined using (2-10).

The downlink expression characterized by (2-21) allows to perform a simplified equalization due to the presence of a diagonal matrix. Note the similarity of (2-21) with (2-9).

\subsection{2}

\section{OFDMA Equalization Strategies}

As supposed, the channel characteristics are kept fixed during the transmission of an OFDMA block, which simplifies the equalization process after the DFT. Each sub-symbol just needs an equalizer of 1 tap, aiming to eliminate the multiplicative distortions caused by the channel. The equalization complexity is pretty simple due to diagonal matrix that characterizes the channel action.

The Zero Forcing (ZF) and Minimum Mean Square Error (MMSE) equalizers assume the ideal knowledge of the propagation channel at reception. Considering the CP addition, symbols with unitary energy and the noise vector $\mathbf{n}$ with a covariance matrix $\mathbf{K}_{n}=\sigma^{2} \mathbf{I}_{M}$, the corresponding matrices to equalizers are given by [1]

$$
\begin{gathered}
\mathbf{A}_{Z F}=\operatorname{Diag}(\mathbf{q})^{-1}, \\
\mathbf{A}_{M M S E}=\operatorname{Diag}(\mathbf{q})^{H}\left[\operatorname{Diag}(\mathbf{q}) \operatorname{Diag}(\mathbf{q})^{H}+\sigma^{2} \mathbf{I}_{M}\right]^{-1},
\end{gathered}
$$

with $\mathbf{A}_{Z F}$ and $\mathbf{A}_{M M S E}$ representing ZF and MMSE matrices, respectively, with dimension $M \times M$. These matrices multiply (2-9) before the detection process, 
which is implemented by minimum distance between the constellation points and the equalized vector components.

\section{2}

\section{Single Carrier Block Transmission - CS-CDMA}

The multiple access technique Chip Spread-CDMA, proposed in [2], performs a combination of blocks transmission, single carrier and the multiple access technique CDMA. As CS-CDMA is based in block transmission, it incorporates a guard interval to avoid the IBI. This guard interval can be of two types: Cyclic Prefix or Zero-Padding (ZP). The main advantage of CSCDMA is allowing the ideal decoupling of the user signals at the receiver even when the signal is propagated through a multipath frequency selective channel $[2]$.

\subsection{1}

\section{CS-CDMA matrix model of transmission and reception}

CS-CDMA system has a strong difference with respect to DS-CDMA. In the CS-CDMA systems, a block of information symbols spreads the chips of the code sequence.This approach expresses an interchange among chips and information symbols in the structure of traditional DS-CDMA systems. With this approach, the new system enables keeping the orthogonality between the transmitted signals of the different users even after the propagation through multipath frequency selective channels. Therefore the signals of the users can be ideally decoupled at the receiver, which is not possible with the traditional DS-CDMA transmission [2].

Synchronous transmission in an uplink time-invariant linear channel with $K$ active users is assumed in this mathematical analysis. The $k$-th user transmits a symbol sequence $\left\{b_{k}(i)\right\}$, which is composed by points in the complex constellation of the adopted modulation scheme. By considering block transmission a block of $N$ symbols, $\mathbf{b}_{k}=\left[b_{k}(0) b_{k}(1) \ldots b_{k}(N-1)\right]^{T}$ is formed. In this new approach, the symbol block is multiplied by the $i$-th chip belonging to spreading sequence of $k$-th user, $c_{k, i}$ formed by $B$ chips. The structure of a CSCDMA system is shown in Figure 2.3. In CS-CDMA systems the maximum number of active users is conditioned by the length of the spread sequence $(K<=B)$, with the aim to keep the orthogonality of codes. Then, to admit more users in the system it is only necessary to use a longer spreading code.

The chips in Figure $2.3\left\{c_{k, i}\right\}_{i=0}^{B-1}$ form the spreading sequence $\mathbf{c}_{k}$ of length $B$ that fulfill the condition $\left\|\mathbf{c}_{k}\right\|^{2}=1$. Then, the transmitted block by the $k$ - 


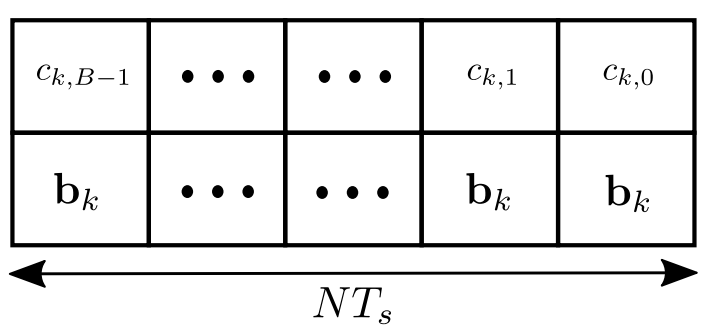

Figure 2.3: Sequence of $B$ transmitted blocks by $k$-th user in CS-CDMA [2].

th user in the $i$-th transmission of a CS-CDMA system is given by $\mathbf{b}_{k} c_{k, i}$ with $(i=0,1,2, \ldots, B-1)$.

After the insertion of the guard interval with $L_{0}$ samples, the resulting block with dimension $M_{0}=N+L_{0}$ is transmitted sequentially through a multipath channel. The impulse response of the discrete channel is given by $\mathbf{h}_{k}=\left[\begin{array}{llll}h_{k, 0} & h_{k, 1} & \ldots & h_{k, P-1}\end{array}\right]^{T}$ of length $P$, where $\mathbb{E}\left[\left\|\mathbf{h}_{k}\right\|^{2}\right]=1$. The transmission through the channel is modeled by means of the Toeplitz matrix $\overline{\mathbf{H}}_{\mathbf{k}}$ with dimension $M_{0} \times M_{0}$, where its first column corresponds to channel impulse response concatenated with zeros,

$$
\mathbf{h}_{k, T e o}=\left[\begin{array}{lllll}
\mathbf{h}_{k} & 0_{1} & \ldots & 0_{M_{0}-P}
\end{array}\right]^{T} .
$$

At reception the detection process depends on the type of guard interval used, which can be CP or ZP. The CS-CDMA system with CP was proposed and analyzed in [7]. The use of ZP is proposed in this work. Both cases are examined in this section and the clear advantage of the $\mathrm{ZP}$ over $\mathrm{CP}$ will become evident.

Assuming the use of Cyclic Prefix and uplink configuration, the RBS receives the information of all users and removes the guard interval of the received blocks. In this way, part of the Toeplitz channel matrix is eliminated resulting in a circulant matrix, $\operatorname{Circ}\left(\overline{\mathbf{h}}_{k}\right)$ with dimension $N \times N$, as explained in Section 2.1.1 concerning the OFDMA processing. The first column of the resultant circulant matrix is defined by the vector,

$$
\mathbf{h}_{k, C i r c}=\left[\begin{array}{lllll}
\mathbf{h}_{k} & 0_{1} & \ldots & 0_{N-P}
\end{array}\right]^{T} .
$$

The receiver obtains the superposition of noisy version signals of $K$ active users after passing through the corresponding channel. Therefore, the $i$-th received block by the RBS can be derived as

$$
\mathbf{r}_{c p}(i)=\sum_{k=1}^{K} \operatorname{Circ}\left(\overline{\mathbf{h}}_{k}\right) \mathbf{b}_{k} c_{k, i}+\mathbf{n}_{c p}(i) \quad i=0,1, \ldots, B-1,
$$

where $\mathbf{n}_{c p}(i)$ represents an additive white Gaussian noise vector with null mean 
and covariance matrix $\mathbb{E}\left[\mathbf{n}_{c p}(i) \mathbf{n}_{c p}^{H}(i)\right]=\sigma^{2} \mathbf{I}_{N}$.

Taking into consideration that the $k$-th user code, $\left\{c_{k, i}\right\}_{i=0}^{B-1}$, is formed by $B$ chips, we can collect $B$ consecutive vectors, $r_{c p}(i), i=0, \ldots, B-1$ and form the observation matrix defined as

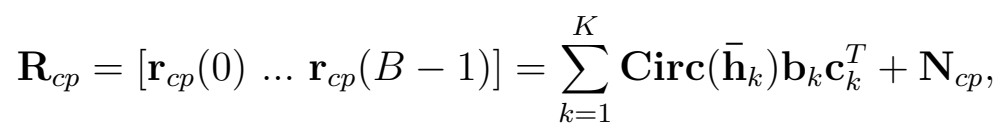

where $\mathbf{N}_{c p}=\left[\mathbf{n}_{c p}(0) \ldots \mathbf{n}_{c p}(B-1)\right]$.

Now, we consider the Zero-Padding guard interval. For this case, in opposite to $\mathrm{CP}$, the guard interval is not discarded at reception. Each one of $B$ transmitted vectors is concatenated with a vector of zeros with length $L_{0}$ at the end of the original vector. The final length of the resultant vector $\left(\mathbf{b}_{k}^{(0)}\right)$ is $M_{0}$. Then the product $\overline{\mathbf{H}}_{k} \mathbf{b}_{k}^{(0)}$ can be replaced by $\overline{\mathbf{H}}_{k}^{(0)} \mathbf{b}_{k}$, where $\overline{\mathbf{H}}_{k}^{(0)}$ is a $M_{0} \times N$ matrix containing the first $N$ columns of $\overline{\mathbf{H}}_{k}$. Then, the received signal by the RBS in uplink configuration is expressed by

$$
\mathbf{r}_{z p}(i)=\sum_{k=1}^{K} \overline{\mathbf{H}}_{\mathbf{k}}^{(\mathbf{0})} \mathbf{b}_{k} c_{k, i}+\mathbf{n}_{z p}(i) \quad i=0,1, \ldots, B-1,
$$

where $\mathbf{n}_{z p}(i)$ represents an additive white Gaussian noise vector with null mean and covariance matrix $\mathbb{E}\left[\mathbf{n}_{z p}(i) \mathbf{n}_{z p}^{H}(i)\right]=\sigma^{2} \mathbf{I}_{N}$. Using the same procedure described for $\mathrm{CP}$, the resulting observation matrix is given by

$$
\mathbf{R}_{z p}=\left[\begin{array}{llll}
\mathbf{r}_{z p}(0) & \ldots & \mathbf{r}_{z p}(B-1)
\end{array}\right]=\sum_{k=1}^{K} \overline{\mathbf{H}}_{\mathbf{k}}^{(\mathbf{0})} \mathbf{b}_{k} \mathbf{c}_{k}^{T}+\mathbf{N}_{z p},
$$

where $\mathbf{N}_{z p}=\left[\mathbf{n}_{z p}(0) \ldots \mathbf{n}_{z p}(B-1)\right]$.

\subsection{2}

\section{CS-CDMA Equalization Strategies}

At reception, different matrices are used in the equalization process of the CS-CDMA signal, according to the employed guard interval. In this section, the equalization process is analyzed for two cases: CP and ZP. The matrix $\mathbf{G}_{k}$ is the channel representation for both guard intervals. In this way, (2-27) and (2-29) can be expressed in an unified way as

$$
\mathbf{R}=\sum_{k=1}^{K} \mathbf{G}_{k} \mathbf{b}_{k} \mathbf{c}_{k}^{T}+\mathbf{N}
$$

where $\mathbf{G}_{k}=\operatorname{Circ}\left(\overline{\mathbf{h}}_{k}\right)$ when $\mathrm{CP}$ is employed as guard interval, while ZP uses $\mathbf{G}_{k}=\overline{\mathbf{H}}_{\mathbf{k}}^{(\mathbf{0})}$. As mentioned before, in both cases the CS-CDMA systems allow the decoupling of the signals of different users at reception ideally. Therefore, the Multiple Access Interference (MAI) is removed even when the transmission is carried out through time dispersive and frequency selective 
channels. The decoupling process can be done before the channel estimation stage (if necessary), equalization and other detection procedures.

For example, considering orthogonal codes where $\mathbf{c}_{m}$ is the spreading sequence to $m$-th user, when the product of matrix $\mathbf{R}$ in (2-30) by the code $\mathbf{c}_{m}$ yields the $m$-th user desired signal

$$
\mathbf{r}_{m}=\mathbf{R c}_{m}=\mathbf{G}_{m} \mathbf{b}_{m}+\mathbf{n}_{m}
$$

where $\mathbf{n}_{m}=\mathbf{N} \mathbf{c}_{m}$ is an additive white Gaussian noise vector with null mean and covariance matrix $\mathbb{E}\left[\mathbf{n}_{m} \mathbf{n}_{m}^{H}\right]=\left\|\mathbf{c}_{m}\right\|^{2} \sigma^{2} \mathbf{I}_{N}=\sigma^{2} \mathbf{I}_{N}$. In (2-31) is evident the MAI absence due to the conservation of signal orthogonality after propagation through multipath channels.

After the separation of the signals pertaining to different users, linear equalizers can be applied with the corresponding matrices given by

$$
\begin{gathered}
\overline{\mathbf{A}}_{Z F}^{(m)}=\mathbf{G}_{m}^{\dagger} \text { and } \\
\overline{\mathbf{A}}_{M M S E}^{(m)}=\mathbf{G}_{m}^{H}\left[\mathbf{G}_{m} \mathbf{G}_{m}^{H}+\sigma^{2} \mathbf{I}\right]^{-1},
\end{gathered}
$$

where $\mathbf{G}_{m}^{\dagger}=\mathbf{G}_{m}^{-1}$ in the $\mathrm{CP}$ case. The channel matrix $\overline{\mathbf{H}}_{\mathbf{m}}^{(\mathbf{0})}$ when the system uses ZP is not square, then $\mathbf{G}_{m}^{\dagger}=\left(\overline{\mathbf{H}}_{\mathbf{m}}^{(\mathbf{0}) \mathbf{H}} \overline{\mathbf{H}}_{\mathbf{m}}^{(\mathbf{0})}\right)^{-1} \overline{\mathbf{H}}_{\mathbf{m}}^{(\mathbf{0}) \mathbf{H}}$ corresponds to the pseudo-inverse. After $\mathbf{r}_{m}$ equalization in (2-31), the detection of $\mathbf{b}_{m}$ components is carried out by minimum distance from signal constellation points to equalized vector components.

\section{3}

\section{Numerical Results: OFDMA and CS-CDMA in time-invariant linear chan- nels}

Aiming at comparing the two multiple access schemes, we consider that both systems have the same equivalent discrete channel impulse response and the same transmission bandwidth. Furthermore, to carry out a fair comparison, the two systems transmit the same number of information symbols $(M=K N)$ and they have the same number of active users in the system. For performance comparison, BER (Bit-Error Rate) curves versus $E_{B} / N_{0}$ in dB were obtained for the techniques described in sections 2.1 and 2.2, where $E_{B}$ denotes the Energy per information bit transmitted.

QPSK modulation and binary Hadamard orthogonal codes of length $B$ were used to generate the curves. The channels associated to the $K$ active users in the system are random, identically distributed, statistically independent, linear and modeled by a time-invariant filter FIR with $P$ coefficients. The coefficients of the $k$-th user channel $\mathbf{h}_{k}$ are given by $h_{k, i}=p_{i} \alpha_{k, i}$, where $\left\{\alpha_{k, i},\right\}_{i=0}^{P-1}$ are statistically independent complex Gaussian random variables 
with zero mean and $\mathbb{E}\left[\left|\alpha_{k, i}\right|^{2}\right]=1$. The values of $\alpha_{k, i}$ are randomly drawn and kept fixed during each simulation run. The weights $p_{i}$ satisfy $\sum_{i=0}^{P-1}\left|p_{i}\right|^{2}=1$. In consequence $\mathbb{E}\left[\left\|\mathbf{h}_{k}\right\|^{2}\right]=1$ and the $p_{i}$ are computed using the relationship $p_{i}=10^{\frac{-i}{P-1}}$ with $i=0,1, . ., P-1$. The guard interval is assumed large enough to allow IBI-free detection i.e. $L \geq P-1$.

Table 2.1 presents the parameters used in the simulations in the case of linear, time-invariant, random and multipath channel.

Table 2.1: Simulation parameters - random, multipath, linear and timeinvariant channel.

\begin{tabular}{lcc}
\hline \hline Parameters & OFDMA & CS-CDMA \\
\hline \hline Modulation & QPSK & QPSK \\
Channel taps $(P)$ & 4 & 4 \\
Sub-carriers $(M)$ & 64,48 & - \\
Data vector length $(N)$ & - & 8,12 \\
Number of channel realizations & 50000 & 50000 \\
Number of information symbols sent & $32 \times 10^{5}$ & $32 \times 10^{5}$ \\
& $24 \times 10^{5}$ & $24 \times 10^{5}$ \\
Users $(K)$ & 8,4 & 8,4 \\
\hline
\end{tabular}

Figures 2.4 and 2.5 illustrate that the use of ZP as guard interval yields better performance results. The matrix that characterizes the channel, $\overline{\mathbf{H}}_{\mathbf{k}}^{(\mathbf{0})}$, is a factor that influences in the results with ZP for uplink configuration. The dimension of the channel matrix is modified to $M_{0} \times N$ (where $M_{0}>N$ ) due to insertion of zeros at the end of the data symbol vector. The interpretation of this fact corresponds to a problem that has more equations than unknowns, allowing to obtain more accurate results. Furthermore, with ZP the energy spent by the system to transmit the symbols inside the guard interval is zero since nothing is transmitted in that interval. In this way, the energy per transmitted symbol is equivalent to the energy per transmitted information symbol, unlike the CP that uses energy to transmit the symbols present in the guard interval. This last reason is evidenced in Figures 2.4 and 2.5 when we can note a little displacement in the curves of CP guard interval.

This analysis is also considered in downlink configuration, where we assume that all channels are equal since the RBS carries out the transmission and each active user receives the transmitted signal through its channel. Figures 2.6 and 2.7 show the previous systems but in downlink configuration.

Figures 2.4, 2.5, 2.6 and 2.7 demonstrate that the use of ZP proposed here is more effective than CP in the CS-CDMA system. For this reason, CS-CDMA systems with ZP as guard interval are the ones considered in the remaining experiments. In CS-CDMA systems, to maintain the orthogonality 


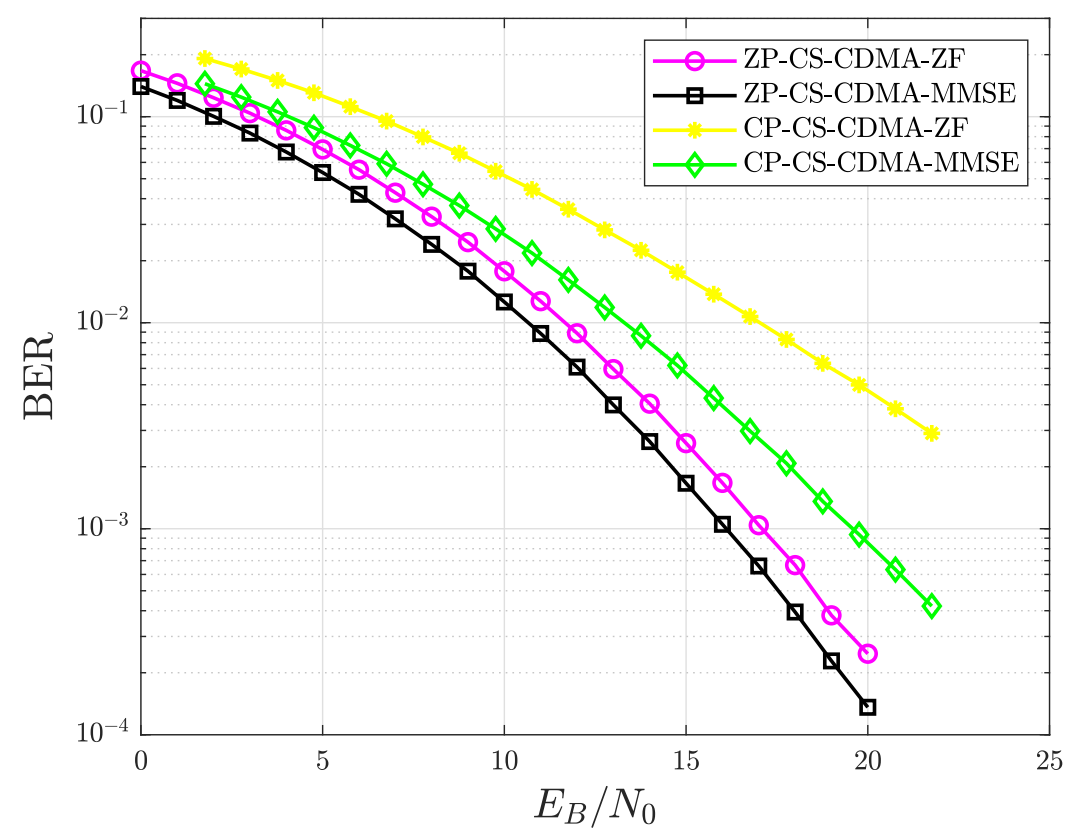

Figure 2.4: BER vs $E_{B} / N_{0}$ curves to CS-CDMA systems with guard interval $\mathrm{ZP}$ and $\mathrm{CP}(L=4), N=8, K=8, B=8$ and $P=4$, in uplink configuration.

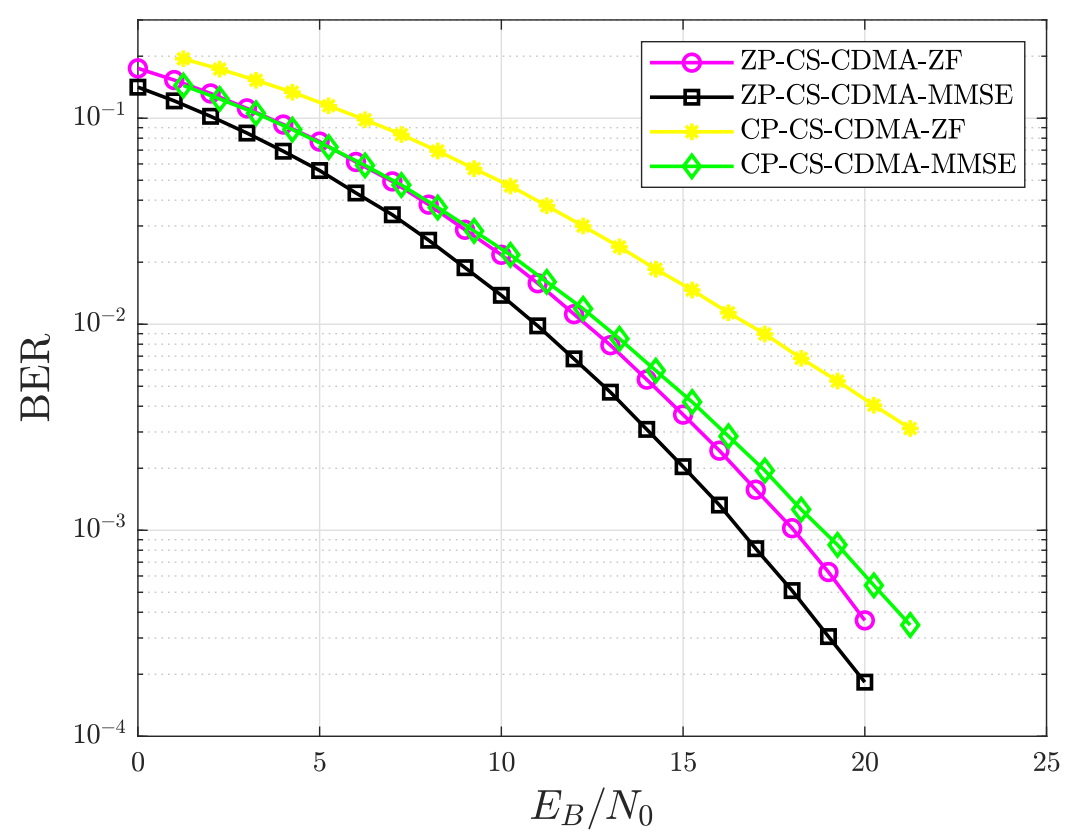

Figure 2.5: BER vs $E_{B} / N_{0}$ curves to CS-CDMA systems with guard interval ZP and $\mathrm{CP}(L=4), N=12, K=4, B=12$ and $P=4$, in uplink configuration.

of the user signals, the length of sequence code should match the maximum number of active users. The parameter set to obtain the simulation results fulfill this condition in the CS-CDMA systems considered. 


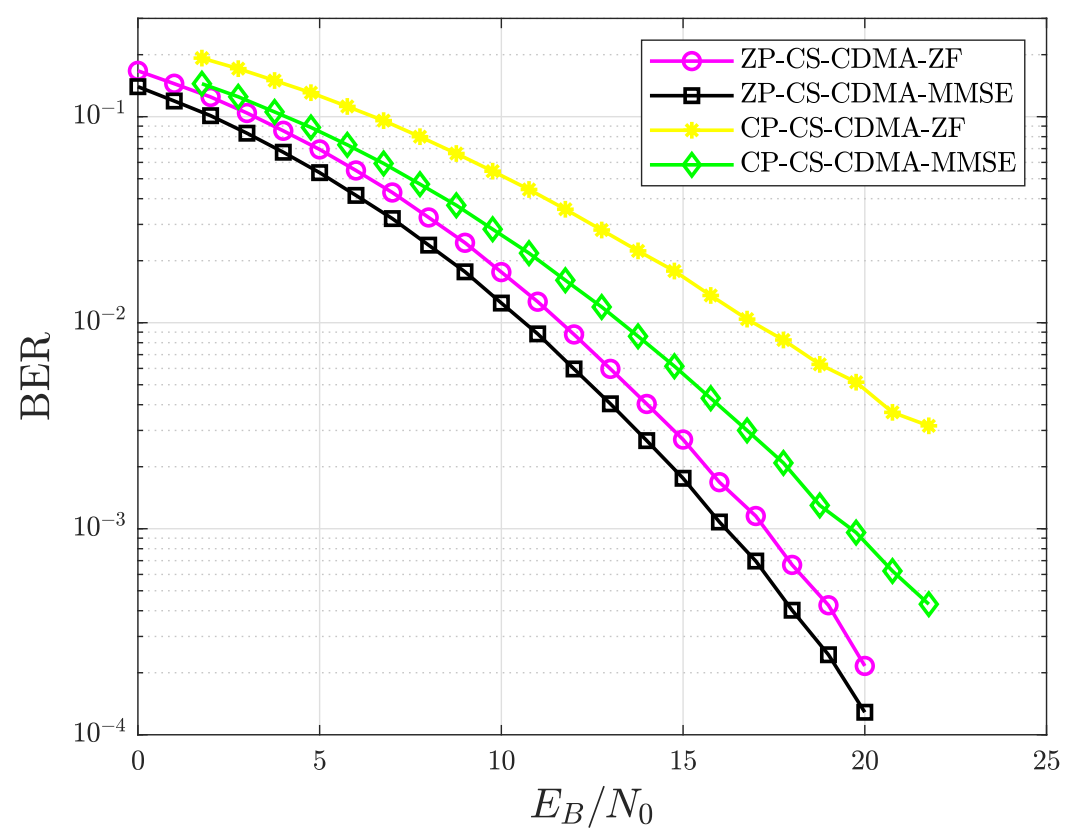

Figure 2.6: BER vs $E_{B} / N_{0}$ curves to CS-CDMA systems with guard interval ZP and $\mathrm{CP}(L=4), N=8, K=8, B=8$ and $P=4$, in downlink configuration.

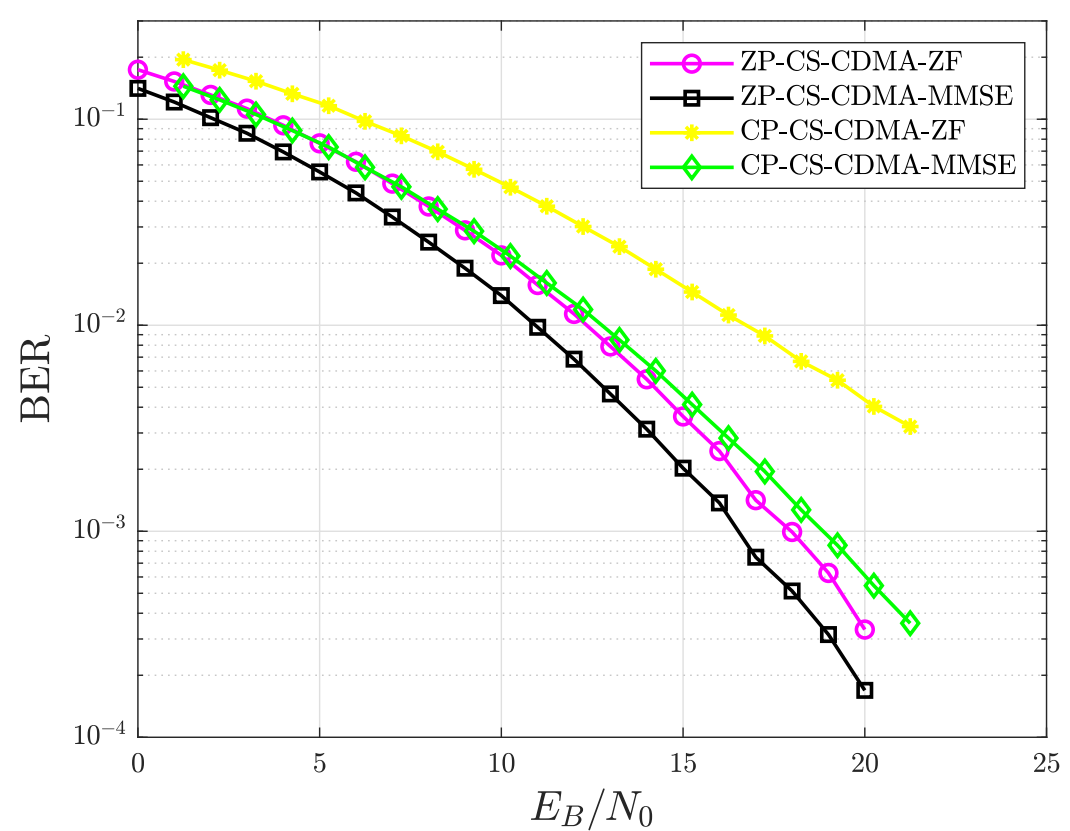

Figure 2.7: BER vs $E_{B} / N_{0}$ curves to CS-CDMA systems with guard interval $\mathrm{ZP}$ and $\mathrm{CP}(L=4), N=12, K=4, B=12$ and $P=4$, in downlink configuration.

Figure 2.8 presents a performance comparison between the OFDMA and CS-CDMA systems, both techniques in uplink configuration. OFDMA 
system is simulated using CP as guard interval, while CS-CDMA uses ZP. This selection induces the little displacement of OFDMA performance curves to the right when expressed as functions of the energy per information bit, $E_{B}$, instead of energy per transmitted bit, $E_{b}$. The displacement here is of approximately $0.263 \mathrm{~dB}$, which is equivalent to $68 / 64$ since this specific system sends 64 information symbols and 4 additional redundant symbols that compose the $\mathrm{CP}$ interval. On the other hand, the CS-CDMA system does not present this issue since the ZP use zeros in the guard interval. Therefore, the system does not spend energy sending the guard interval.

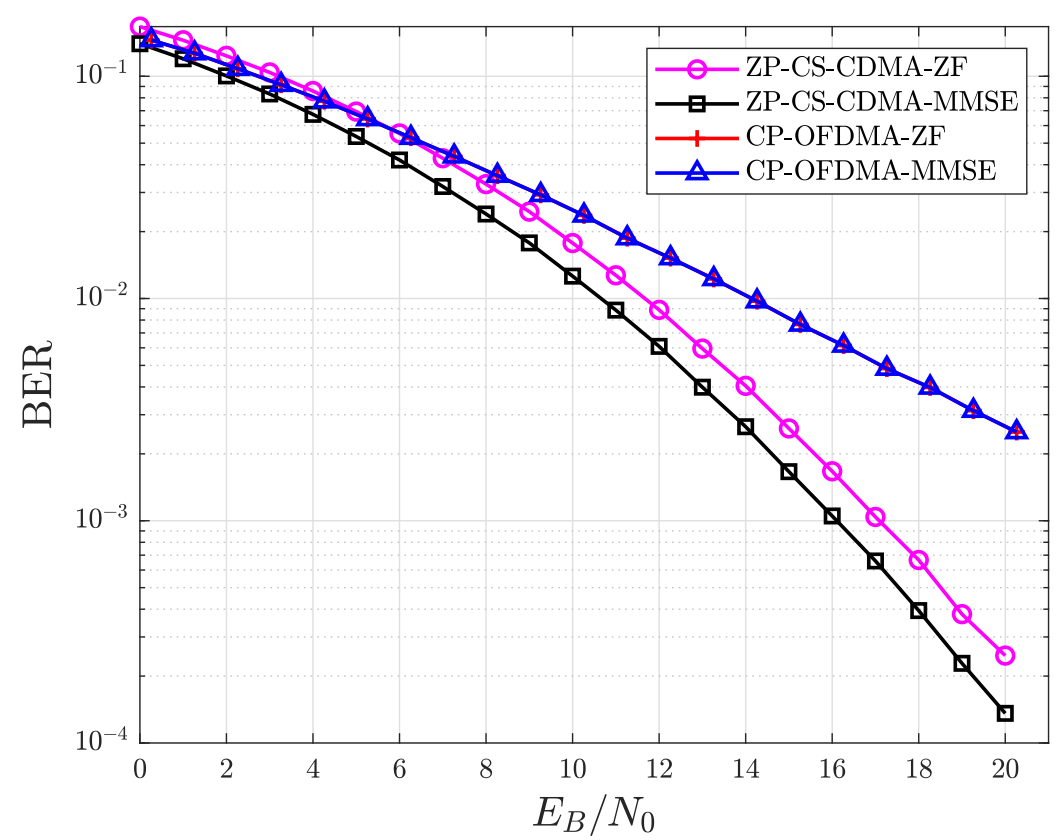

Figure 2.8: BER vs $E_{B} / N_{0}$ curves of OFDMA and CS-CDMA systems with a set of parameters $L=4, M=64, N=8, K=8, B=8$ and $P=4$, in uplink configuration.

Figure 2.9 illustrates the performance with another parameter set in uplink configuration and the curves behavior is maintained. Indeed, OFDMA performance curves are independent of the number of subcarriers and the number of taps of the channel (see Appendix B). In this case the displacement of OFDMA curves is of approximately $0.347 \mathrm{~dB}$ since the system sends 48 information symbols and 4 redundant symbols in the guard interval.

The downlink configuration was also analyzed and the simulation results for the same sets of parameters of figures 2.8 and 2.9 are presented in figures 2.10 and 2.11, respectively. It is evident in all simulations the wide superiority in terms of performance of the CS-CDMA technique over OFDMA. Although, it should be mentioned that when ZP-CS-CDMA is used, the system suffers a 


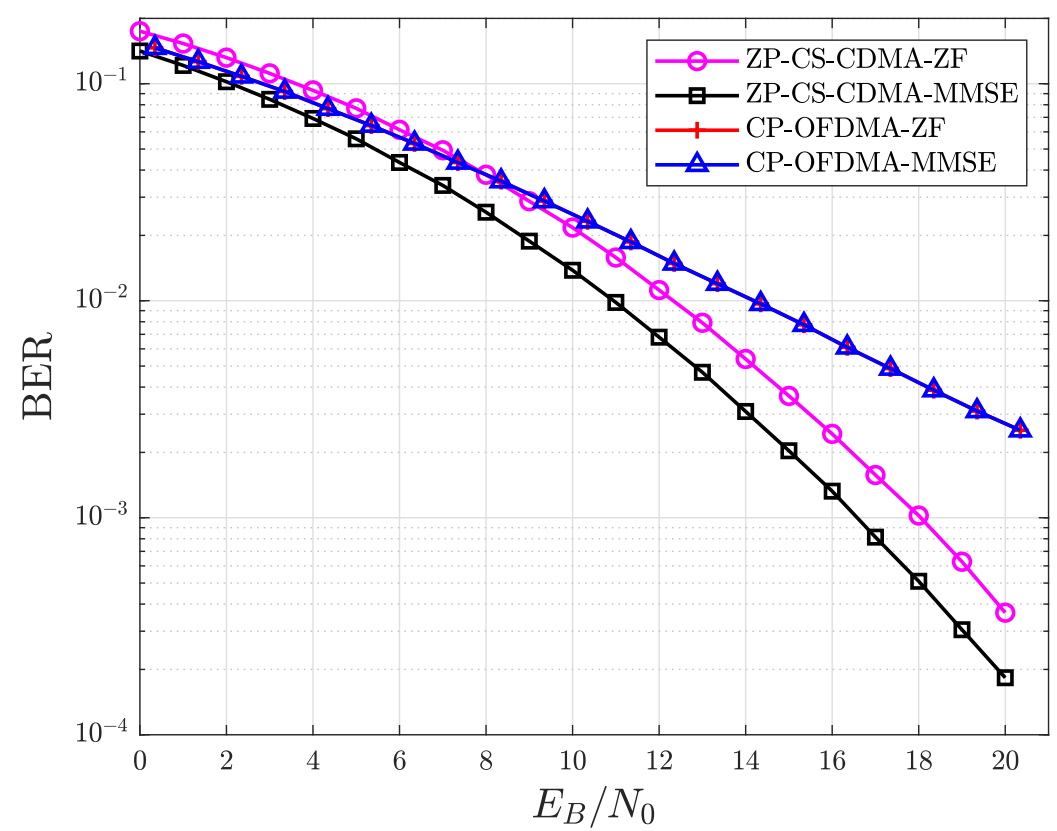

Figure 2.9: BER vs $E_{B} / N_{0}$ curves of OFDMA and CS-CDMA systems with a set of parameters $L=4, M=48, N=12, K=4, B=12$ and $P=4$, in uplink configuration.

small loss of spectral efficiency. This issue is treated in more details in Section 2.4 .

\section{4 Spectral Analysis}

The spectral analysis deals with the shape and occupation of the spectrum of a given signal. This procedure provides an important aspect for comparison between the two considered techniques in sections 2.1 and 2.2. The analysis refers to the output of the transmitter, being independent of the channel characteristics.

To obtain a general expression for Power Spectral Density (PSD), the Appendix A presents a mathematical development to compute the PSD expression of any block transmission system with blocks of signals sent continuously by the transmitter. The expression depends only on the transmission pulse $g(t)$ and the autocorrelation matrix of the transmitted vector $\boldsymbol{\chi}$, the corresponding PSD is given by

$$
\mathbf{S}_{x}(f)=R_{s}|G(f)|^{2} \frac{1}{D} \sum_{l=0}^{D-1} \sum_{n=0}^{D-1} R_{\chi}(l, n) e^{-j 2 \pi(l-n) f / R_{s}}
$$

where $R_{\chi}(l, n)$ are the autocorrelation matrix components of the transmitted block. The length of the transmitted vector is $D \times 1$ and it is considered that 


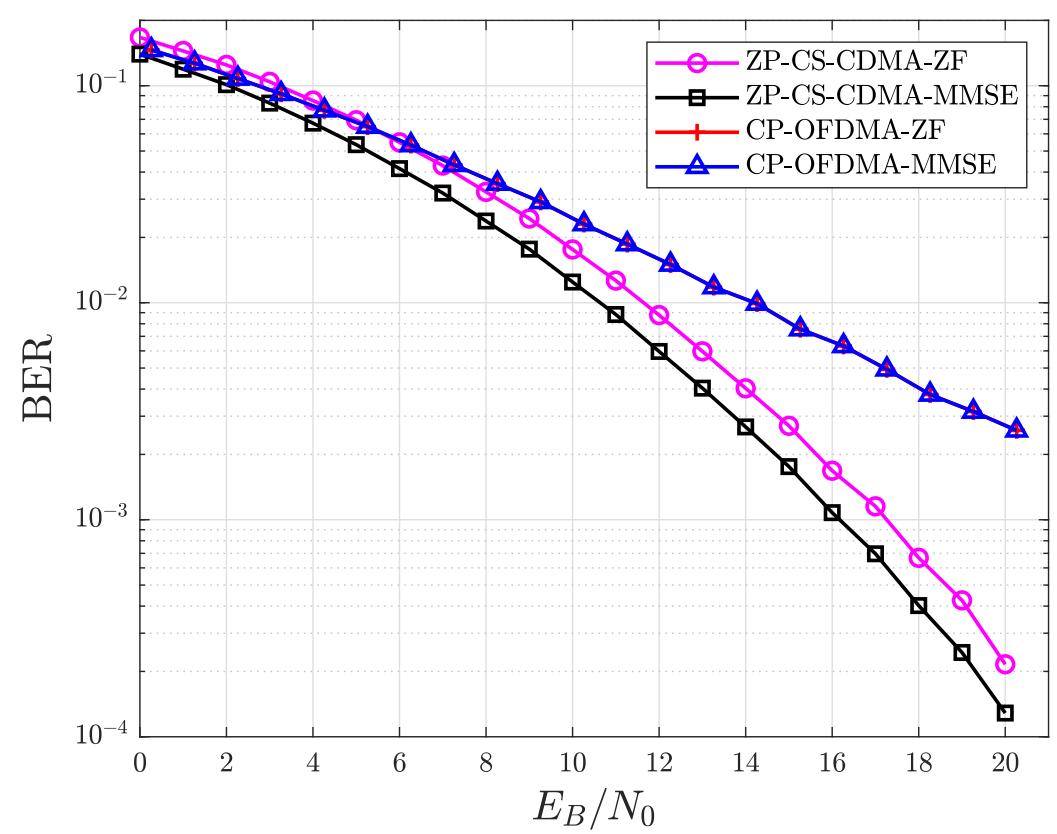

Figure 2.10: BER vs $E_{B} / N_{0}$ curves of OFDMA and CS-CDMA systems with a set of parameters $L=4, M=64, N=8, K=8, B=8$ and $P=4$, in downlink configuration.

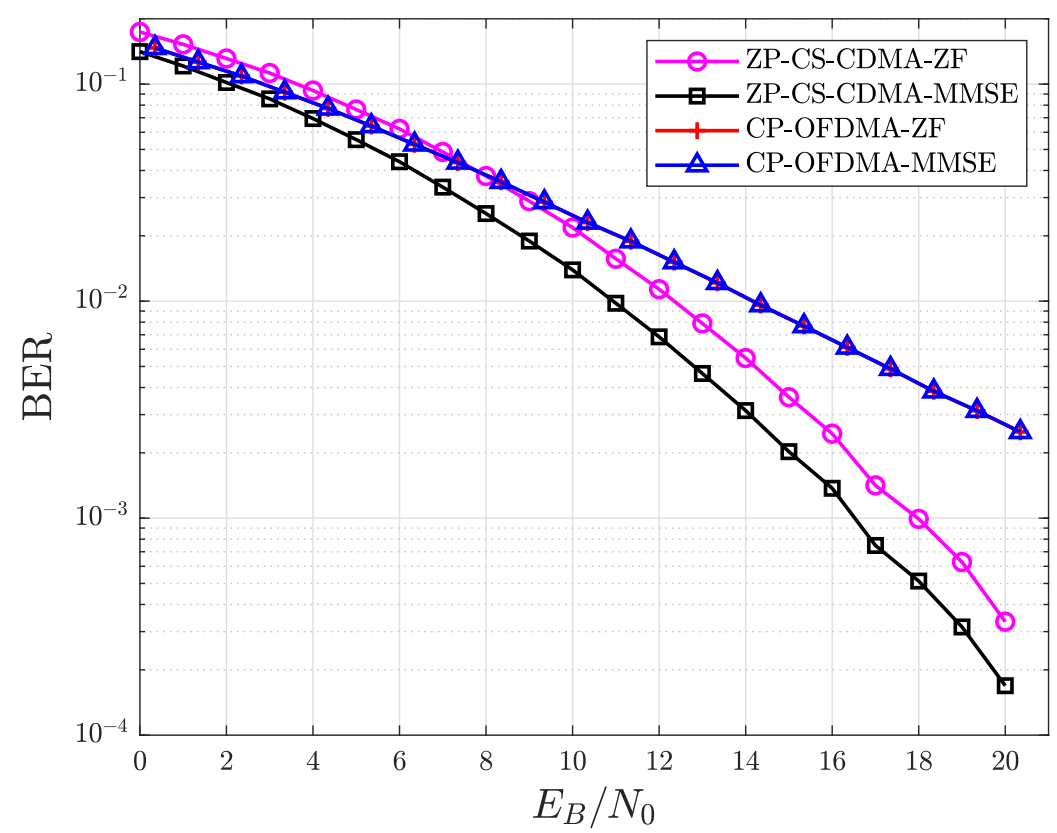

Figure 2.11: BER vs $E_{B} / N_{0}$ curves of OFDMA and CS-CDMA systems with a set of parameters $L=4, M=48, N=12, K=4, B=12$ and $P=4$, in downlink configuration. 
$\chi^{(i)}=\boldsymbol{\Psi} \mathbf{d}_{i}$, where $\boldsymbol{\Psi}$ is the transmission matrix with dimension $D \times Z$ and $\mathbf{d}_{i}$ depicts the $Z \times 1$ vector that contains uncorrelated data symbols.

The complex components of $\chi^{(i)}$ are sent at a symbol rate $R_{s}$, using a phase-quadrature transmission scheme and a transmission pulse $g(t)$ with spectrum $G(f)$.

The autocorrelation matrix of transmitted vector $\chi^{(i)}$ is

$$
\mathbf{R}_{\boldsymbol{\chi}}=\mathbb{E}\left[\chi^{(i)} \chi^{(i) H}\right]=\sigma_{d}^{2} \boldsymbol{\Psi} \Psi^{H}
$$

where $\sigma_{d}^{2}$ is the variance of the components of $\mathbf{d}_{i}$. To evaluate (2-35), we consider the specific transmissions matrix of the block transmission system and compute its PSD using (2-34). This was done for the two techniques studied in this work: OFDMA and CS-CDMA. The analysis is divided in two parts, with and without guard interval.

\subsection{1}

\section{Systems without guard interval}

In this subsection, the transmission is performed without guard interval. For better understanding, OFDMA and CS-CDMA are examined independently. The main objective is to find the expression that characterizes the transmission matrix $\Psi$.

\subsubsection{1}

\section{OFDMA}

Following the previous procedure, the PSD for an OFDMA block transmission system can be computed employing (2-34). The vector in this case is expressed as

$$
\chi^{(i)}=\mathbf{W}_{M}^{H} \mathbf{d}_{i}
$$

where $\boldsymbol{\Psi}=\mathbf{W}_{M}^{H}$ and $\mathbf{d}_{i}$ are the transmission matrix (IDFT matrix) with dimension $M \times M$ and data symbols with length $M \times 1$, respectively.

By substituting the transmission matrix in (2-35), we have the autocorrelation matrix of transmitted vector

$$
\mathbf{R}_{\boldsymbol{\chi}}=\sigma_{d}^{2} \mathbf{W}_{M}^{H} \mathbf{W}_{M}=\sigma_{d}^{2} \mathbf{I}_{M}
$$

Then, using the result in (2-37) and substituting in (2-34), the PSD expression of OFDMA is reduced to

$$
\mathbf{S}_{x}(f)=\frac{\sigma_{d}^{2}}{T_{s}}|G(f)|^{2}
$$

To check the results, Figure 2.12 illustrates the result of the multiplication of a IDFT and DFT matrices generated with dimension 64. This result as 
expected is due to the fact that the two matrices are orthogonal by returning the identity matrix.

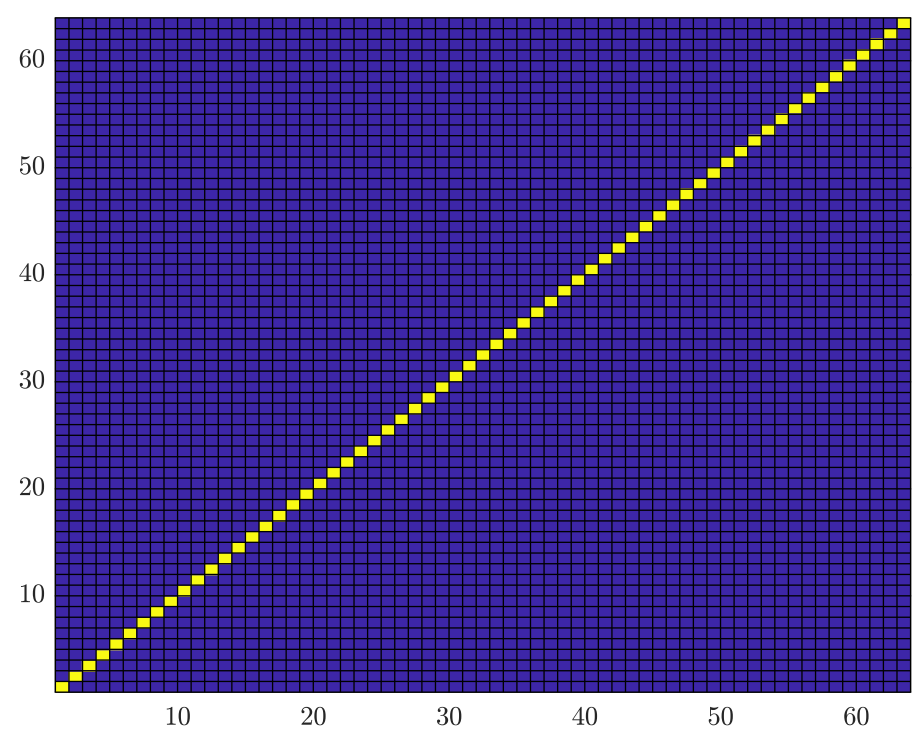

Figure 2.12: Transmission matrices multiplication $\boldsymbol{\Psi} \Psi^{H}$ of OFDMA with $M=64$ and without guard interval.

\subsubsection{2}

\section{CS-CDMA}

As part of the comparison, the PSD corresponding to a CS-CDMA system without guard interval is computed. Applying the same previous procedure, the first step is to find the transmission matrix.

In CS-CDMA, the transmitted vector corresponding to $k$-th user $\chi_{k}$ employs the code $\mathbf{c}_{k}=\left(\begin{array}{lllll}c_{k 1} & c_{k 2} & \ldots & c_{k N}\end{array}\right)^{T}$. This vector can be expressed as

$$
\boldsymbol{\chi}_{k}=\left[\begin{array}{c}
c_{k 1} \mathbf{b}_{k} \\
c_{k 2} \mathbf{b}_{k} \\
\vdots \\
c_{k N} \mathbf{b}_{k}
\end{array}\right]=\underbrace{\left[\begin{array}{c}
c_{k 1} \mathbf{I}_{N} \\
c_{k 2} \mathbf{I}_{N} \\
\vdots \\
c_{k N} \mathbf{I}_{N}
\end{array}\right]}_{\mathbf{C}_{\mathbf{k}}} \mathbf{b}_{k}=\mathbf{C}_{k} \mathbf{b}_{k} .
$$

In (2-39), it is evident that the guard interval is not present in the $k$-th user vector. Considering $K$ active users, the total transmitted vector is given by 


$$
\chi=\mathbf{C}_{1} \mathbf{b}_{1}+\mathbf{C}_{2} \mathbf{b}_{2}+\ldots+\mathbf{C}_{K} \mathbf{b}_{K}=\underbrace{\left(\begin{array}{llll}
\mathbf{C}_{1} & \mathbf{C}_{2} & \ldots & \mathbf{C}_{K}
\end{array}\right)}_{\Psi}\left[\begin{array}{c}
\mathbf{b}_{1} \\
\mathbf{b}_{2} \\
\vdots \\
\mathbf{b}_{K}
\end{array}\right]=\Psi \mathbf{b}
$$

where $\mathbf{b}$ represents the total data vector with dimension $K N \times 1$ formed by the vertical concatenation of $K$ data vectors. The transmission matrix $\boldsymbol{\Psi}$ has a dimension $K N \times K N$.

In CS-CDMA, the transmission matrix is a concatenation of $K$ transmission matrices. In this work, the Hadamard code is used to generate the code sequence of each user (see Section 2.2). The codes assigned to users are orthogonal. Substituting the transmission matrix in (2-35), we arrive at

$$
\mathbf{R}_{\boldsymbol{\chi}}=\sigma_{d}^{2}\left(\begin{array}{llllll}
\mathbf{C}_{1} & \mathbf{C}_{2} & \ldots & \mathbf{C}_{K}
\end{array}\right)\left(\begin{array}{lllll}
\mathbf{C}_{1} & \mathbf{C}_{2} & \ldots & \mathbf{C}_{K}
\end{array}\right)^{H}=\sigma_{d}^{2} \sum_{i=1}^{K} \mathbf{C}_{i} \mathbf{C}_{i}^{H}=\sigma_{d}^{2} \mathbf{I}_{K N}
$$

By substituting the autocorrelation matrix in (2-34), we obtain the PSD equation of CS-CDMA system without guard interval

$$
\mathbf{S}_{x}(f)=\frac{\sigma_{d}^{2}}{T_{s}}|G(f)|^{2}
$$

The PSD equation in (2-42) is the same expression obtained to OFDMA in (2-38), both cases without guard interval. Figure 2.13 illustrates the computer generated product $\Psi \Psi^{H}$ corresponding to a CS-CDMA system with 8 users $(K=8)$ each one transmitting 8 data symbols $(N=8)$ and a code sequence of length $8(B=8)$.

\subsubsection{3}

\section{Numerical results of OFDMA and CS-CDMA without guard interval}

In this subsection, the results of PSD for OFDMA and CS-CDMA without guard interval are presented. The computation employs (2-38) and 2-42 to obtain the PSD corresponding to both techniques. Rectangular transmission pulses with duration $T_{s}$ were considered. The spectrum of this pulse is given by

$$
G(f)=\operatorname{sinc}\left(f / R_{s}\right) .
$$

where $\operatorname{sinc}(x)=\frac{\operatorname{sen}(\pi x)}{\pi x}$. Here we note that for comparisons involving different systems, it is more meaningful to express the PSD in terms of the data symbol rate $R_{D}$ that may differ from the transmitted symbol rate $R_{s}$. If the guard intervals are not considered then $R_{s}=R_{D}$.

Figure 2.14 shows the PSD computed for the OFDMA and CS-CDMA systems without guard interval, with parameters $M=64, N=8, K=8$ and 


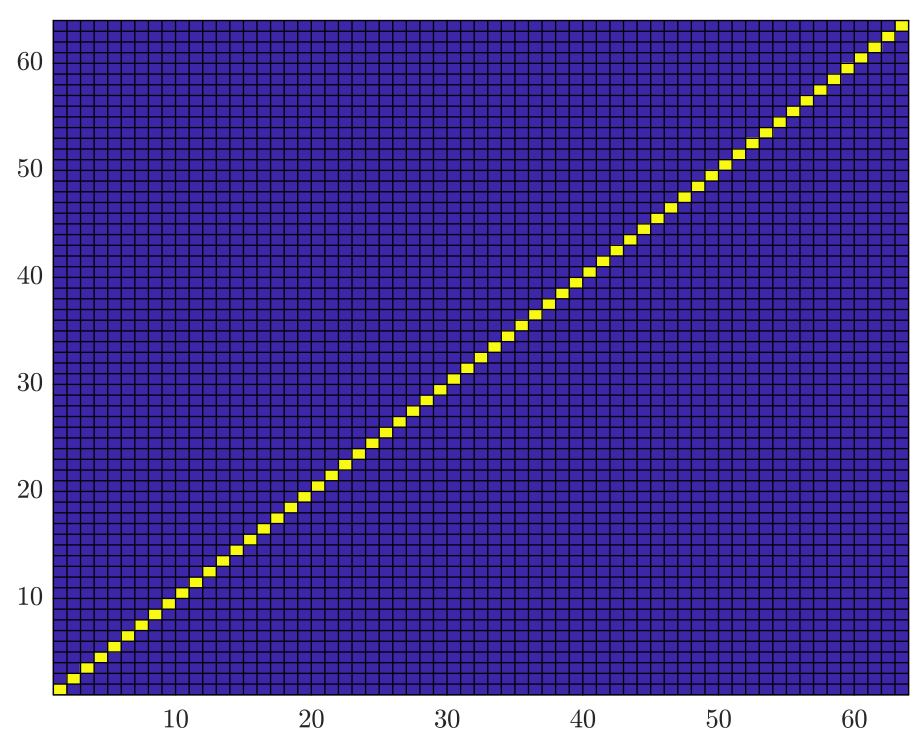

Figure 2.13: Transmission matrices multiplication $\boldsymbol{\Psi} \Psi^{H}$ of CS-CDMA with $N=8, K=8, B=8$ and without guard interval.

$B=8$. This parameter set was selected to obtain an equivalence between the systems. In Figure 2.14 the spectrum is normalized by its maximum value.

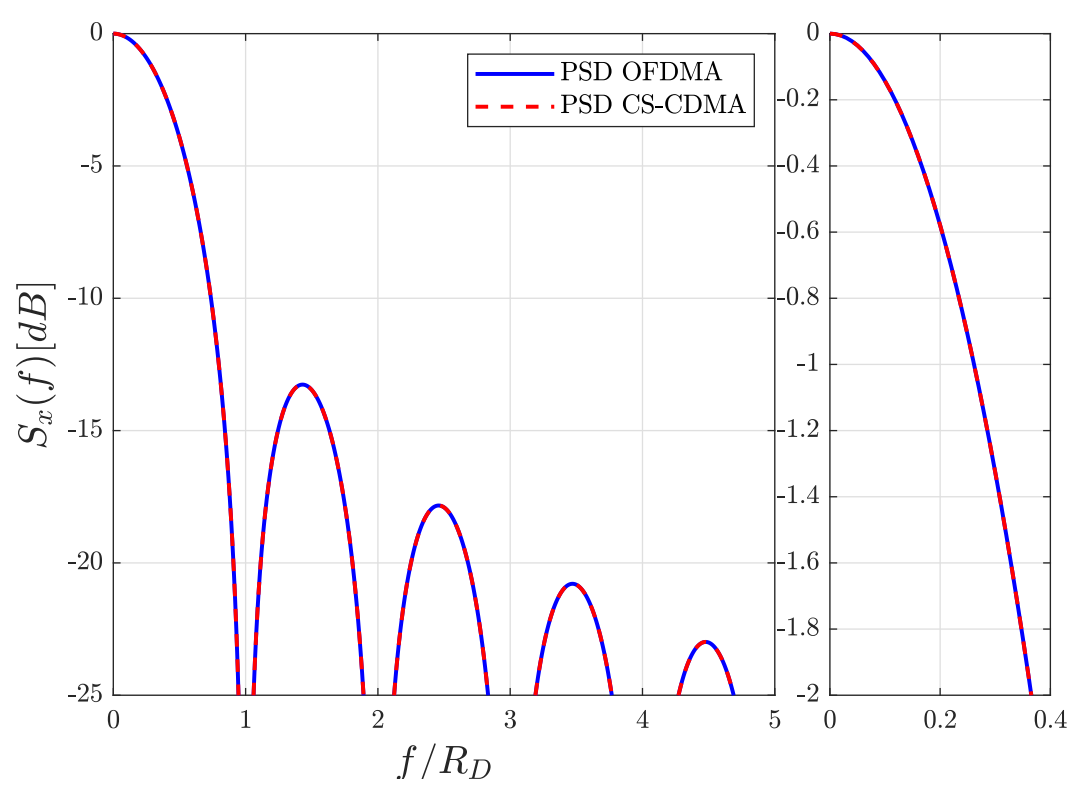

Figure 2.14: Power Spectral Density of OFDMA system with FFT $64(M=64)$ and CS-CDMA system with $N=8, K=8$ and $B=8$, both without guard interval normalized by the maximum point $\left(R_{D}\right.$ is the data symbol rate).

Another way of normalizing the results is by the area. Figure 2.15 
shows the PSD of OFDMA and CS-CDMA systems with the same parameters employed in Figure 2.14.

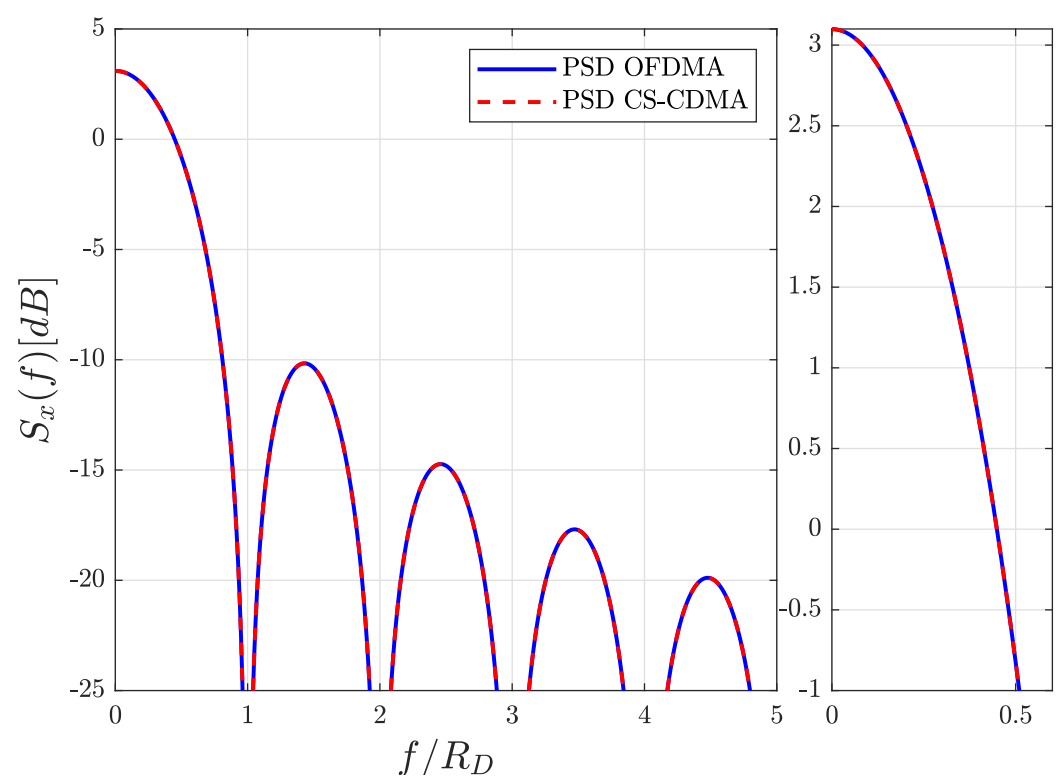

Figure 2.15: Power Spectral Density of OFDMA system with FFT $64(M=64)$ and CS-CDMA system with $N=8, K=8$ and $B=8$, both without guard interval normalized by area.

It is interesting to include in the spectral analysis a representation of the percentage of the total emission power out of a given frequency band B. Figure 2.16 presents the curves corresponding to both techniques, where the two systems have the same behavior with respect to the out of band emission, approximately $9 \%$ in a band $\mathrm{B}=R_{D}$.

\section{4 .2}

Systems with guard interval

In this part, the PSD of the systems with the presence of a guard interval is analyzed. The OFDMA system uses Cyclic Prefix and to CS-CDMA the Zero Padding method. The main objective is the same as in the previous section: to find the transmission matrix $\boldsymbol{\Psi}$, the autocorrelation matrix $\boldsymbol{\Psi} \boldsymbol{\Psi}^{H}$ and obtain the PSD of each system via (2-35) and (2-34).

\subsubsection{1}

\section{CP-OFDMA}

In the case of OFDMA using $\mathrm{CP}$, the last $L$ rows of the IDFT matrix are placed in the top of the matrix as commented in Section 2.1.1. In this way, the matrix $\mathbf{M}$ is defined, which performs the insertion of the CP. Repeating 


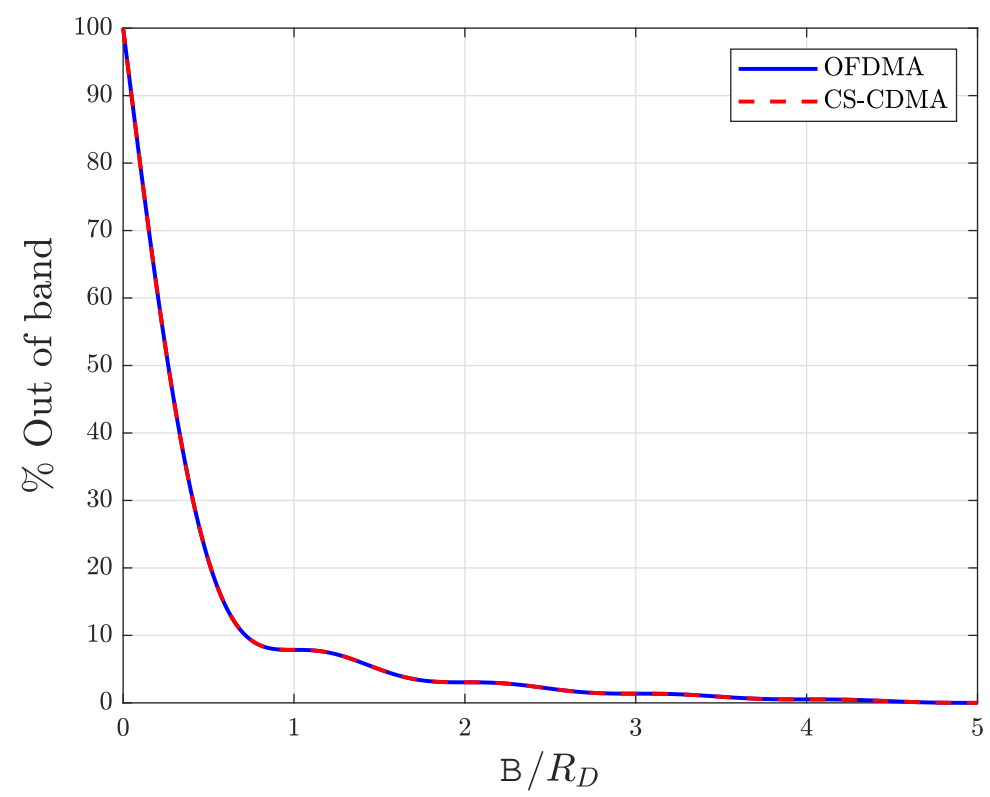

Figure 2.16: Percentage out of band: OFDMA system with FFT $64(M=64)$ and CS-CDMA system with $N=8, K=8$ and $B=8$.

the expression of $\mathbf{M}$ in (2-3) for convenience, we have that

$$
\mathbf{M}=\left[\begin{array}{c}
\mathbf{W}_{c p}^{H} \\
\mathbf{W}_{M}^{H}
\end{array}\right],
$$

where $\mathbf{W}_{c p}^{H}$ is formed by the last $L$ rows of $\mathbf{W}_{M}^{H}$. The dimension of $\mathbf{M}$ is $(M+L) \times M$. Then, the CP-OFDMA transmitted vector can be written as

$$
\chi^{(i)}=\mathbf{M d}_{i}
$$

and the transmission matrix is in this case $\boldsymbol{\Psi}=\mathbf{M}$ and from (2-35) the autocorrelation matrix is given by

$$
\mathbf{R}_{\chi}=\sigma_{d}^{2} \mathbf{M M}^{H}
$$

Figure 2.17 illustrates the values of the matrix $\boldsymbol{\Psi} \Psi^{H}$ and the effects of the inclusion of the CP. These effects are manifested in the values different from zero near the corners. The parameter set used to generate Figure 2.17 was $M=64$ and $L=4$.

\subsubsection{2 ZP-CS-CDMA}

In the case of ZP-CS-CDMA, the Zero Padding strategy was implemented to eliminate the IBI. With ZP, it is necessary to create $L$ new rows of zeros at the end of the identity matrices used to compute the transmission vector 


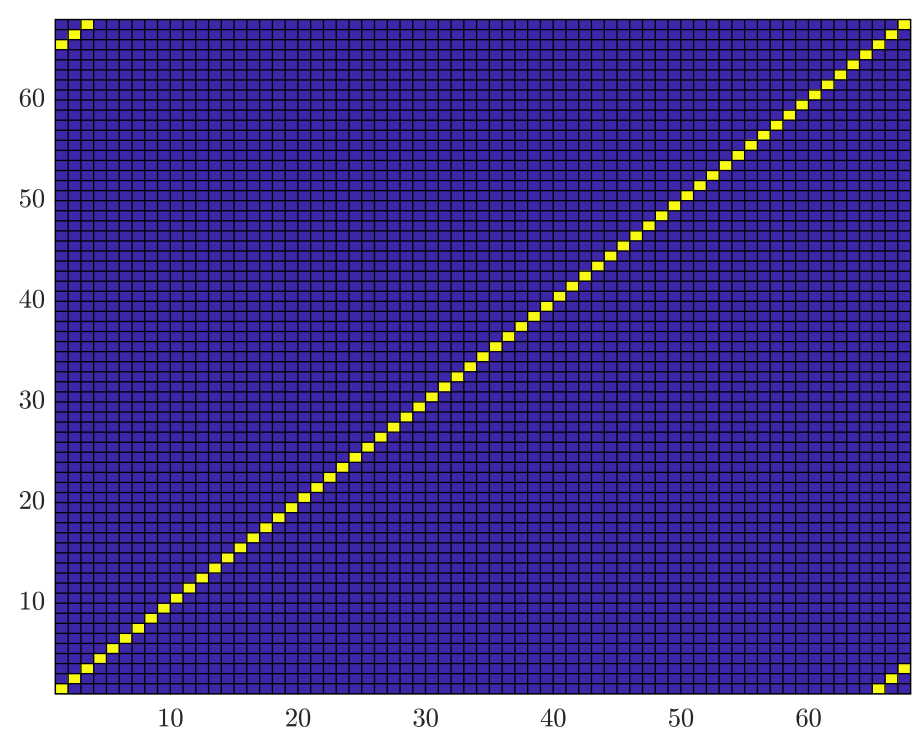

Figure 2.17: Transmission matrices multiplication $\boldsymbol{\Psi} \boldsymbol{\Psi}^{H}$ of CP-OFDMA with $M=64$ and $L=4$.

corresponding to each user. The transmission vector of the $k$-th user is defined as

$$
\boldsymbol{\chi}_{k}=\left[\begin{array}{c}
c_{k 1} \mathbf{b}_{k}^{(0)} \\
c_{k 2} \mathbf{b}_{k}^{(0)} \\
\vdots \\
c_{k N} \mathbf{b}_{k}^{(0)}
\end{array}\right]=\left[\begin{array}{c}
c_{k 1} \mathbf{I}_{Z P} \\
c_{k 2} \mathbf{I}_{Z P} \\
\vdots \\
c_{k N} \mathbf{I}_{Z P}
\end{array}\right] \mathbf{b}_{k}^{(0)}=\mathbf{C}_{k}^{Z P} \mathbf{b}_{k}^{(0)},
$$

where by the ZP effect, $\mathbf{b}_{k}^{(0)}=\left[\begin{array}{c}\mathbf{b}_{k} \\ \mathbf{0}_{L \times 1}\end{array}\right]$ with dimension $(N+L) \times 1, \mathbf{0}_{L \times 1}$ is a vector of zeros with length $L \times 1, \mathbf{I}_{Z P}=\left[\begin{array}{c}\mathbf{I}_{N} \\ \mathbf{0}_{N \times P}\end{array}\right]$ and $\mathbf{0}_{N \times P}$ is a matrix of zeros with dimension $N \times P$.

Considering $K$ active users the transmitted vector $\chi$ with the form

$\chi=\mathbf{C}_{1}^{Z P} \mathbf{b}_{1}^{(0)}+\mathbf{C}_{2}^{Z P} \mathbf{b}_{2}^{(0)}+\ldots+\mathbf{C}_{K}^{Z P} \mathbf{b}_{K}^{(0)}=\underbrace{\left(\begin{array}{llll}\mathbf{C}_{1}^{Z P} & \mathbf{C}_{2}^{Z P} & \ldots & \mathbf{C}_{K}^{Z P}\end{array}\right)}_{\Psi}\left[\begin{array}{c}\mathbf{b}_{1}^{(0)} \\ \mathbf{b}_{2}^{(0)} \\ \vdots \\ \mathbf{b}_{K}^{(0)}\end{array}\right]=\mathbf{\Psi} \mathbf{b}^{(0)}$,

where $\mathbf{b}^{(0)}$ is a column vector with dimension $(N+L) K \times 1$ since each vector $\mathbf{b}_{k}^{(0)}$ contains $L$ zeros at the end. This fact is a disadvantage of CS-CDMA compared to OFDMA, the block created by CS-CDMA has $K L$ additional zeros, which has impact in its spectral efficiency and power spectral density.

Figure 2.18 shows the result of transmission matrix multiplication $\Psi \Psi^{H}$ 
with $N=8, B=8$ and $K=8$. The obtained matrix was affected by the ZP use, obviously. These effects are illustrated with the presence of zeros in the main diagonal.

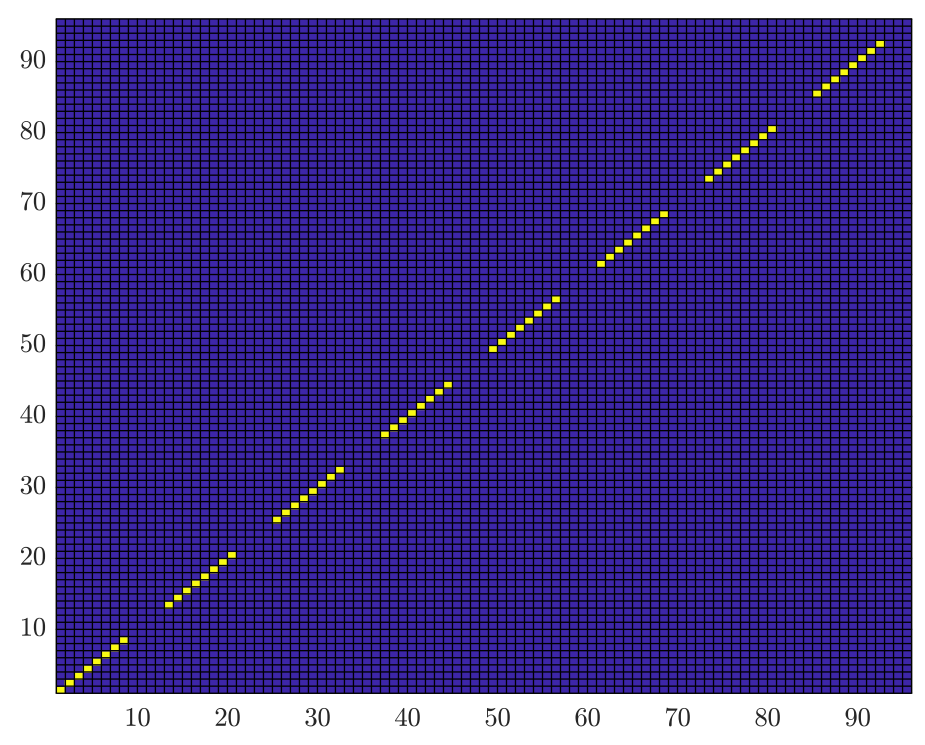

Figure 2.18: Transmission matrices multiplication $\boldsymbol{\Psi} \boldsymbol{\Psi}^{H}$ of ZP-CS-CDMA with $N=8, K=8, B=8$.

Using (2-35) with the corresponding transmission matrix, the autocorrelation matrix can be expressed by

$$
\mathbf{R}_{\boldsymbol{\chi}}=\sigma_{d}^{2} \underbrace{\left(\begin{array}{llll}
\mathbf{C}_{1}^{Z P} & \mathbf{C}_{2}^{Z P} & \ldots & \mathbf{C}_{K}^{Z P}
\end{array}\right)}_{\mathbf{C}}\left(\begin{array}{llll}
\mathbf{C}_{1}^{Z P} & \mathbf{C}_{2}^{Z P} & \ldots & \mathbf{C}_{K}^{Z P}
\end{array}\right)^{H}=\sigma_{d}^{2} \mathbf{C C}^{H}
$$

\subsubsection{3}

\section{Numerical results of OFDMA and CS-CDMA with guard interval}

As noted before, in CS-CDMA systems a block of NK data symbols generates a block of $(N+L) K$ transmitted symbols (zeros included), corresponding to a spectral efficiency of $\eta_{C D M A}=(N K) /(N+L) K$. The relationship between the symbol transmission rate $R_{s}$ and data symbol rate, $R_{D}$, is in this case

$$
R_{s}=\frac{1}{\eta_{C D M A}} R_{D}=\frac{N+L}{N} R_{D}
$$

For the OFDMA system we have $\eta_{O F D M A}=(N K) /(N K+L)$ and

$$
R_{s}=\frac{N K+L}{N K} R_{D}
$$

the advantage of the OFDMA system in terms of spectral efficiency is by a factor of 


$$
\frac{\eta_{O F D M A}}{\eta_{C D M A}}=\frac{(N+L) K}{N K+L} .
$$

The power spectral densities are presented in terms of the data symbol rate $R_{D}$, using the relations (2-50) and (2-51) in expressions (2-34) and (2-43) for the ZP-CS-CDMA and OFDMA systems, respectively.

Figure 2.19 shows PSD results for CP-OFDMA and ZP-CS-CDMA, using the normalization by the point of maximum. The parameters used in this simulation were $M=64, N=8, K=8, B=8$ and $L=4$.

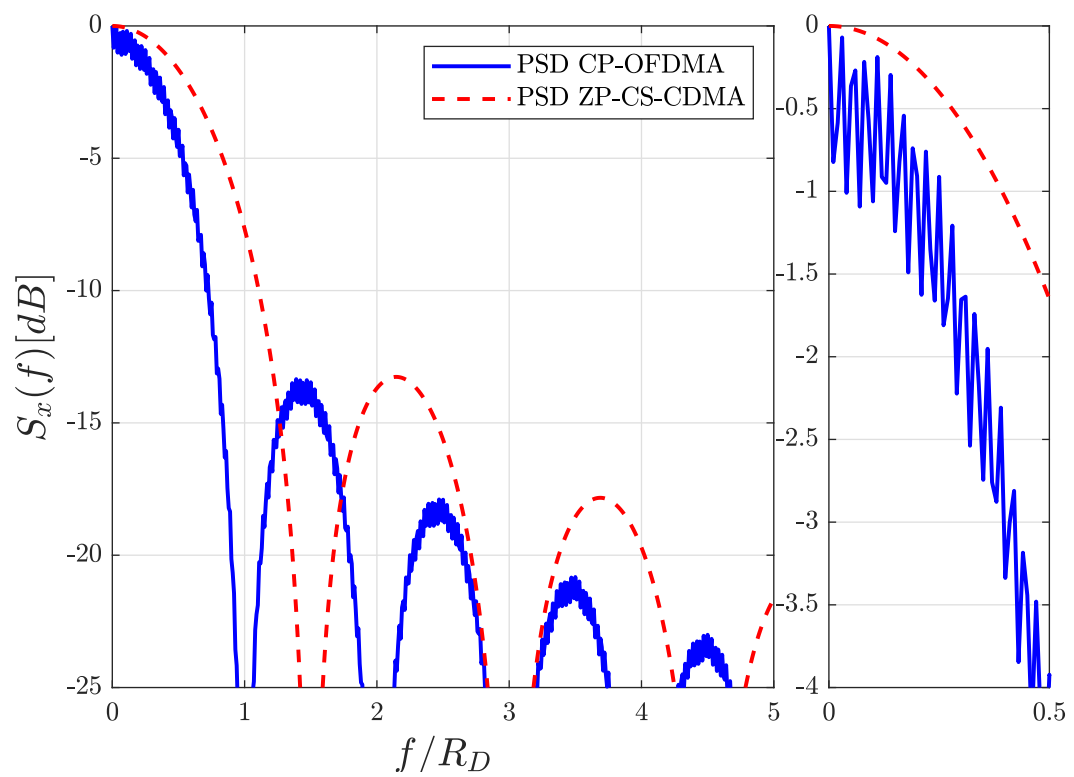

Figure 2.19: Power Spectral Density of CP-OFDMA system with FFT 64 $(M=64)$ and ZP-CS-CDMA system with $N=8, K=8$ and $B=8$, both with a guard interval of $L=4$ and normalized by the maximum point.

The widening of the ZP-CS-CDMA is clearly defined and the disadvantage with respect to the spectral occupation of ZP-CS-CDMA becomes apparent. Figure 2.20 illustrates the simulation results using the normalization by the area. The parameter set used in this experiment is the same employed in the previous normalization.

In both Figures 2.19 and 2.20 the PSD of the CP-CDMA shows ripples due the presence of the cyclic prefix. The percentage of the emission power out of a given band B is an important aspect for comparison. Figure 2.21 presents results obtained with the two techniques, evidencing that the percentage of transmission out of band of ZP-CS-CDMA is higher than CP-OFDMA for the same information rate. For a frequency band half the information symbol rate, for example, the values are $\sim 40 \%$ and $20 \%$ for the CS-CDMA and OFDMA cases, respectively. 


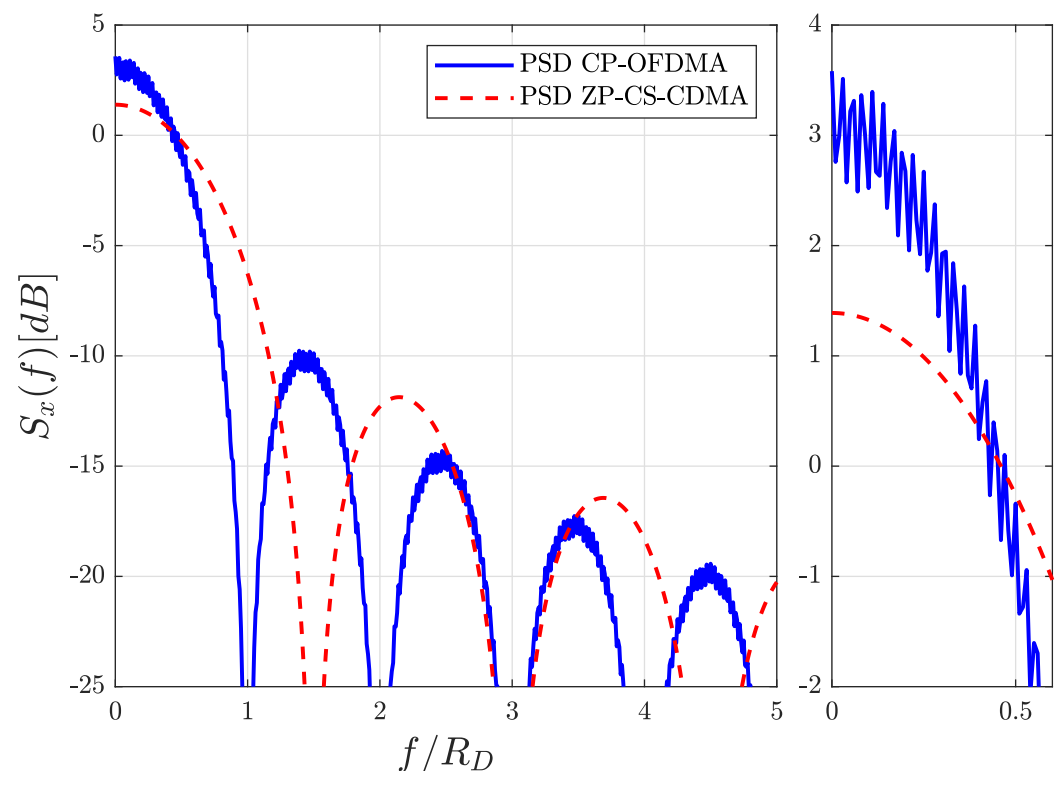

Figure 2.20: Power Spectral Density of CP-OFDMA system with FFT 64 $(M=64)$ and ZP-CS-CDMA system with $N=8, K=8$ and $T=8$, both with a guard interval of $L=4$ and normalized by the area.

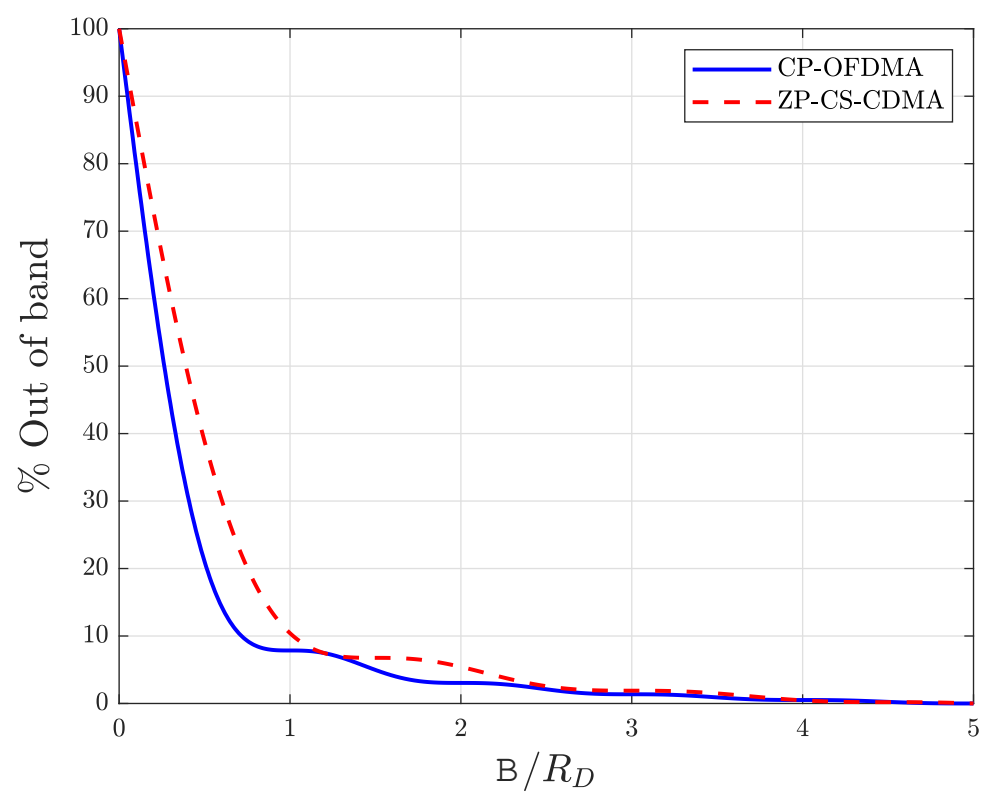

Figure 2.21: Percentage out of band to CP-OFDMA system with FFT 64 $(M=64)$ and ZP-CS-CDMA system with $N=8, K=8$ and $B=8$, and both with $L=4$. 


\section{Block Transmission Schemes in time-varying linear channels}

In this chapter, the effect of fast variations of the channel in block transmission systems is discussed. In the previous chapter, a linear and semistatic channel was assumed, in other words, the channel remains constant during the transmission of a block of symbols. Now, the channel varies from symbol to symbol within a transmission block. The effects associated with this condition are assessed in this chapter.

\section{1}

\section{OFDMA in time-varying linear environments}

In Section 2.1.1, the matrix model of the OFDMA system when the signal is transmitted through a time-invariant linear channel was studied. In this section, the propagation in time-varying linear environments is evaluated. The propagation channel can vary from symbol to symbol inside a symbol block, therefore, the matrix $\mathbf{H}(i)$ that describes the channel behavior is not given by a Toeplitz matrix. The channel matrix is composed of impulse responses that vary with each column of the matrix. Hence, the elements do not repeat along the diagonals of the matrix [17]. The channel matrix $\mathbf{H}(i)$ is defined as

$$
\mathbf{H}(i)=\left[\begin{array}{ccccccc}
h_{0}^{(i G)} & 0 & \ldots & 0 & \ldots & 0 & 0 \\
\vdots & h_{0}^{(i G+1)} & \ddots & 0 & \ddots & 0 & 0 \\
h_{L-2}^{(i G)} & \vdots & \ddots & \ddots & \ddots & 0 & 0 \\
h_{L-1}^{(i G)} & h_{L-2}^{(i G+1)} & \ddots & \ddots & \ddots & \vdots & 0 \\
0 & h_{L-1}^{(i G+1)} & \ddots & \ddots & \ddots & 0 & \vdots \\
\vdots & \ddots & \ddots & \ddots & \ddots & h_{0}^{(i G+G-2)} & 0 \\
0 & \ldots & \ldots & \ldots & \ldots & h_{1}^{(i G+G-2)} & h_{0}^{(i G+G-1)}
\end{array}\right]_{G \times G}
$$

The effects of the matrix $\mathbf{H}_{i b i}$ corresponding to IBI are eliminated by the use of CP, as explained in Section 2.1.1. Then, the received signal in the absence of the IBI term is given by

$$
\begin{aligned}
\mathbf{r}_{c p}(i) & =\mathbf{H}(i) \mathbf{M}_{c p} \mathbf{d}(i)+\mathbf{n}_{c p}(i) \\
& =\mathbf{V}_{c p}(i) \mathbf{d}(i)+\mathbf{n}_{c p}(i)
\end{aligned}
$$


where $\mathbf{M}_{c p}$ was defined in (2-3) with dimension $G \times M$ and $\mathbf{n}_{c p}(i)$ represents a $G \times 1$ white Gaussian noise vector. Analogously to the semi-static model, the $\mathrm{CP}$ is removed yielding the observation vector

$$
\mathbf{r}(i)=\left[\mathbf{0}_{M \times L} \mathbf{I}_{M}\right] \mathbf{r}_{c p}(i)=\mathbf{V}(i) \mathbf{d}(i)+\mathbf{n}(i),
$$

where $\mathbf{V}(i)$ is a $M \times M$ matrix and $\mathbf{n}(i)$ is a $M \times 1$ vector.

After the application of the DFT matrix, $\mathbf{W}_{M}$, in (3-3), the received signal is expressed by

$$
\begin{aligned}
\mathbf{x}(i) & =\mathbf{W}_{M} \mathbf{V}(i) \mathbf{d}(i)+\mathbf{W}_{M} \mathbf{n}(i) \\
& =\mathbf{B}_{i} \mathbf{d}(i)+\mathbf{W}_{M} \mathbf{n}(i) \\
& =\mathbf{B}_{i} \mathbf{d}(i)+\tilde{\mathbf{n}}(i),
\end{aligned}
$$

where $\mathbf{B}_{i}=\mathbf{W}_{M} \mathbf{V}(i)$ and $\tilde{\mathbf{n}}(i)=\mathbf{W}_{M} \mathbf{n}(i)$, which maintains the original statistics of $\mathbf{n}(i)$.

Since $\mathbf{H}(i)$ in (3-1) is not a Toeplitz matrix, the channel matrix $\mathbf{B}_{i}$ is no longer a diagonal matrix. This fact complicates the processing to detect the information vector $\mathbf{d}(i)$.

Assuming as an example, a system with two active users in uplink configuration, (3-4) can be rewritten as

$$
\mathbf{x}(i)=\mathbf{B}_{1} \mathbf{d}_{1}+\mathbf{B}_{2} \mathbf{d}_{2}+\tilde{\mathbf{n}}(i),
$$

where $\mathbf{B}_{1}$ and $\mathbf{B}_{2}$ are the channel matrices corresponding to each user, while $\mathbf{d}_{1}$ and $\mathbf{d}_{2}$ are the data symbol vectors sent by users with dimension $M \times 1$. Each data symbol vector is composed by $\tilde{\mathbf{d}}_{1}$ and $\tilde{\mathbf{d}}_{2}$, respectively, and zeros in the correct places depending on corresponding user. Thus (3-5) can be redefined as

$$
\mathbf{x}(i)=\mathbf{B}_{1}\left[\begin{array}{c}
\tilde{\mathbf{d}}_{1} \\
0 \\
\vdots \\
0_{M-J}
\end{array}\right]+\mathbf{B}_{2}\left[\begin{array}{c}
0 \\
\vdots \\
0_{M-J} \\
\tilde{\mathbf{d}}_{2}
\end{array}\right]+\tilde{\mathbf{n}}(i)
$$

where $J \leq \frac{M}{2}$. The expression (3-6) can be simplified by suppressing the zeros

$$
\mathbf{x}(i)=\tilde{\mathbf{B}}_{1} \tilde{\mathbf{d}}_{1}+\tilde{\mathbf{B}}_{2} \tilde{\mathbf{d}}_{2}+\tilde{\mathbf{n}}(i),
$$

where $\tilde{\mathbf{B}}_{1}$ is composed by the first $J$ columns of $\mathbf{B}_{1}$ and $\tilde{\mathbf{B}}_{2}$ contains the last $J$ columns of $\mathbf{B}_{2}$. Compacting (3-7) yields

$$
\mathbf{x}(i)=\mathbb{B}\left[\begin{array}{c}
\tilde{\mathbf{d}}_{1} \\
\tilde{\mathbf{d}}_{2}
\end{array}\right]+\tilde{\mathbf{n}}(i),
$$

where $\mathbb{B}=\left[\tilde{\mathbf{B}}_{1} \mid \tilde{\mathbf{B}}_{2}\right]$ is a matrix formed by two horizontally concatenated 
matrices, $\tilde{\mathbf{B}}_{1}$ and $\tilde{\mathbf{B}}_{2}$. In the downlink configuration, the $\mathbf{B}_{i}$ matrices are equal. The equalization process uses (3-8) and applies two well-known algorithms, Zero Forcing and Minimum Mean Square Error.

\subsection{1}

\section{OFDMA with Zero Forcing Equalization}

Zero Forcing $(\mathrm{ZF})$ is an equalization technique that processes the received signal to undo the effects of the channel on the transmitted signal, regardless of disturbances such as the additive noise $[18,19]$. In this way, the ISI is reduced and the equalizer uses the Moore-Penrose pseudo-inverse matrix. With these concepts, the expression that characterizes the ZF equalizer is given by

$$
\mathbf{A}_{Z F}=\left(\mathbb{B}^{H} \mathbb{B}\right)^{-1} \mathbb{B}^{H},
$$

where the equalized signal is expressed as

$$
\mathbf{y}_{Z F}=\mathbf{A}_{Z F} \mathbf{x}
$$

Substituting (3-8) and (3-9) in (3-10), we have that

$$
\begin{aligned}
\mathbf{y}_{Z F} & =\left(\mathbb{B}^{H} \mathbb{B}\right)^{-1} \mathbb{B}^{H} \mathbb{B}\left[\begin{array}{l}
\tilde{\mathbf{d}}_{1} \\
\tilde{\mathbf{d}}_{2}
\end{array}\right]+\left(\mathbb{B}^{H} \mathbb{B}\right)^{-1} \mathbb{B}^{H} \tilde{\mathbf{n}} \\
& =\left[\begin{array}{c}
\tilde{\mathbf{d}}_{1} \\
\tilde{\mathbf{d}}_{2}
\end{array}\right]+\tilde{\mathbf{n}}_{B},
\end{aligned}
$$

where $\tilde{\mathbf{n}}_{B}=\left(\mathbb{B}^{H} \mathbb{B}\right)^{-1} \mathbb{B}^{H} \tilde{\mathbf{n}}$. In this way, the symbols can be detected using a minimum distance receiver. When the determinant of the matrix $\left(\mathbb{B}^{H} \mathbb{B}\right)$ assumes small values, the additive noise power is amplified, resulting in a worse performance [20].

\subsection{2}

\section{OFDMA with Minimum Mean Square Error Equalization}

The MMSE equalizer is an expansion of ZF that takes into account the statistical characteristics of the Gaussian noise during the matrix inversion step. The method consists in minimizing the mean square error between the received signal vector $\mathbf{y}_{M M S E}$ and the data vector, maximizing the sum of the signal-to-noise ratio with the interference [20]. The expression that represents the MMSE equalization is

$$
\mathbf{A}_{M M S E}=\arg \min _{\mathbf{A}} \mathbb{E}\left[\left\|\left[\begin{array}{l}
\tilde{\mathbf{d}}_{1} \\
\tilde{\mathbf{d}}_{2}
\end{array}\right]-\mathbf{A} \tilde{\mathbf{z}}_{m}\right\|^{2}\right],
$$

which provides 


$$
\mathbf{A}_{M M S E}=\left(\mathbb{B}^{H} \mathbb{B}+\sigma^{2} \mathbf{I}_{M}\right)^{-1} \mathbb{B}^{H} .
$$

The equalized signal is expressed as

$$
\mathbf{y}_{M M S E}=\mathbf{A}_{M M S E} \mathbf{x} .
$$

Substituting (3-8) and (3-13) in (3-14), we obtain

$$
\mathbf{y}_{M M S E}=\left(\mathbb{B}^{H} \mathbb{B}+\sigma^{2} \mathbf{I}_{M}\right)^{-1} \mathbb{B}^{H} \mathbb{B}\left[\begin{array}{c}
\tilde{\mathbf{d}}_{1} \\
\tilde{\mathbf{d}}_{2}
\end{array}\right]+\left(\mathbb{B}^{H} \mathbb{B}+\sigma^{2} \mathbf{I}_{M}\right)^{-1} \mathbb{B}^{H} \tilde{\mathbf{n}},
$$

and the data symbol vector is estimated using a minimum distance receiver in accordance with the signal constellation.

\section{2}

\section{CS-CDMA in time-varying linear environments}

The characterization of the CS-CDMA systems in time-invariant linear channels was discussed in Section 2.2. In this section, we analyze the CSCDMA systems in time-varying linear environments, where there is a relative offset between the transmitter and receiver. This offset induces a deviation in the frequency, called the Doppler effect, which is proportional to the relative velocity of displacement [21]. In both techniques: OFDMA and CS-CDMA, the time-varying channels analyzed in this chapter are based on the Clarke model $[22,23]$.

As explained in Section 2.2, the block transmitted in the CS-CDMA scheme by the $k$-th user in the $i$-th transmission is given by $\mathbf{b}_{k} c_{k, i}$ with $i=0,1, . ., B-1$, where $c_{k, i}$ is the $i$-th chip of the sequence $\mathbf{c}_{k}$.

The received vector in time-varying linear channels can be modeled in a similar way to invariant channels (2-28). The signal received by the RBS in an uplink configuration is given by

$$
\mathbf{r}(i)=\sum_{k=1}^{K} \overline{\mathbf{H}}_{\mathbf{k}}^{(\mathbf{0})}(i) \mathbf{b}_{k} c_{k, i}+\mathbf{n}_{N}(i) \quad i=0,1, \ldots, B-1,
$$

where $\mathbf{n}_{N}(i)$ is a complex Gaussian noise vector with null mean and covariance matrix $\mathbf{K}_{n}=\sigma^{2} \mathbf{I}_{N}$.

The received vector $\mathbf{r}(i)$ is multiplied by the $i$-th code chip of the $m$ th active user. The equalization process corresponding to the $m$-th user is 
performed after the reception of $B$ chips and is expressed by

$$
\begin{aligned}
\mathbf{z}_{m} & =\sum_{i=1}^{B} \mathbf{r}(i) c_{m, i} \\
& =\sum_{i=1}^{B} \overline{\mathbf{H}}_{\mathbf{m}}^{(\mathbf{0})}(i) \mathbf{b}_{m} \underbrace{c_{m, i} c_{m, i}}_{\frac{1}{B}}+\sum_{\substack{j=1 \\
j \neq m}}^{K} \sum_{i=1}^{B} \overline{\mathbf{H}}_{\mathbf{j}}^{(\mathbf{0})}(i) \mathbf{b}_{j} c_{j, i} c_{m, i}+\sum_{i=1}^{B} \mathbf{n}(i) c_{m, i} \\
& =\underbrace{\frac{1}{B} \sum_{i=1}^{B} \overline{\mathbf{H}}_{\mathbf{m}}^{(\mathbf{0})}(i)}_{\mathbb{H}_{m, m}} \mathbf{b}_{m}+\sum_{\substack{j=1 \\
j \neq m}}^{\frac{1}{B} \sum_{i=1}^{B} \overline{\mathbf{H}}_{\mathbf{j}}^{(\mathbf{0})}(i) B c_{j, i} c_{m, i}} \mathbf{b}_{j}+\sum_{i=1}^{B} \mathbf{n}(i) c_{m, i} \\
& =\mathbb{H}_{m, m} \mathbf{b}_{m}+\sum_{\substack{j=1 \\
j \neq m}}^{K} \mathbb{H}_{j, m} \mathbf{b}_{j}+\tilde{\mathbf{n}}_{m},
\end{aligned}
$$

where the matrix $\mathbb{H}_{m, m}$ is a matrix associated to the desired user given by the mean of the channel matrices along the $B$ transmissions. $\mathbb{H}_{j, m}$ is a matrix obtained by the weighted mean (weighted by the values \pm 1 of product $B c_{j, i} c_{m, i}$ ) of the channel matrices associated with the interfering users and $\tilde{\mathbf{n}}_{m}$ is a Gaussian noise vector with null mean and covariance matrix $\mathbf{K}_{\tilde{\mathbf{n}}_{m}}=\sigma^{2} \mathbf{I}_{N}$. The desired user vector is defined by

$$
\tilde{\mathbf{z}}_{m}=\mathbb{H}_{m, m} \mathbf{b}_{m}+\mathrm{MAI}+\tilde{\mathbf{n}}_{m}
$$

where MAI represents the multiple access interference caused by the remaining active users. The time-invariant linear channels are a particular case of (3-17) where $\overline{\mathbf{H}}_{\mathbf{k}}^{(\mathbf{0})}(i)=\overline{\mathbf{H}}_{\mathbf{k}}^{(\mathbf{0})}$, we then have that $\mathbb{H}_{m, m}=\overline{\mathbf{H}}_{\mathbf{m}}^{(\mathbf{0})}$ and $\mathbb{H}_{j, m}=\overline{\mathbf{H}}_{\mathbf{j}}^{(\mathbf{0})} \mathbf{c}_{j}^{T} \mathbf{c}_{u}$. Using orthogonal codes, MAI $=0$ and therefore, (3-18) matches with (2-31). The equation (3-17) shows that in time-varying linear channels the CS-CDMA systems do not remove completely the MAI.

In downlink configuration, the channel matrices associated to each user are assumed equal. Finally, applying the equalization techniques ZF and MMSE, and the minimum distance detection, the vectors of the data symbols are estimated.

\subsection{1}

\section{CS-CDMA with Zero Forcing Equalization}

Assuming that $m$ is the index of the user of interest and considering (3-17), the equalization matrix corresponding to ZF is expressed as:

$$
\mathbf{A}_{Z F}^{m}=\mathbb{H}_{m, m}^{\dagger}
$$

where $(.)^{\dagger}$ is the pseudo-inverse operator. This operation is necessary since $\mathbb{H}_{m, m}$ is not a square matrix. 
After the equalization, the block with $N$ information symbols is detected as

$$
\begin{aligned}
\mathbf{y}_{Z F} & =\mathbf{A}_{Z F}^{m} \tilde{\mathbf{z}}_{m} \\
& =\mathbf{A}_{Z F}^{m}\left[\mathbb{H}_{m, m} \mathbf{b}_{m}+\mathrm{MAI}+\tilde{\mathbf{n}}_{m}\right] \\
& =\mathbf{b}_{m}+\mathrm{MAI}_{A}+\tilde{\mathbf{n}}_{A},
\end{aligned}
$$

where $\operatorname{MAI}_{A}=\mathbf{A}_{Z F}^{m}$ MAI and $\tilde{\mathbf{n}}_{A}=\mathbf{A}_{Z F}^{m} \tilde{\mathbf{n}}_{m}$. After the equalization process, the components of the data symbols of the desired user $\left(\mathbf{b}_{m}\right)$ are detected using the algorithm of minimum distance.

\subsection{2}

\section{CS-CDMA with Minimum Mean Square Error Equalization}

The MMSE equalizer corresponding to signal $\tilde{\mathbf{z}}_{m}$ is defined by $\mathbf{A}_{M M S E}^{m}$ with dimension $N \times N+L$. This matrix is obtained by minimizing the mean square error function

$$
\mathbf{A}_{M M S E}^{m}=\arg \min _{\mathbf{A}} \mathbb{E}\left[\left\|\mathbf{b}_{m}-\mathbf{A} \tilde{\mathbf{z}}_{m}\right\|^{2}\right],
$$

which is solved through

$$
\mathbf{A}_{M M S E}^{m}=\mathbb{H}_{m, m}^{H} \mathrm{R}_{m}^{-1},
$$

where $\mathrm{R}_{m}$ is the autocorrelation matrix of $\tilde{\mathbf{z}}_{m}$, such that

$$
\mathrm{R}_{m}=\mathbb{H}_{m, m} \mathbb{H}_{m, m}^{H}+\sum_{\substack{j=1 \\ j \neq m}}^{K} \mathbb{H}_{j, m} \mathbb{H}_{j, m}^{H}+\sigma^{2} \mathbf{I}_{N}
$$

When the environment is time-invariant and the codes are orthogonal, CS-CDMA can ideally detect in absence of MAI and the matrix is simplified to $\mathrm{R}_{m}^{o}=\left[\mathbb{H}_{m, m} \mathbb{H}_{m, m}^{H}+\sigma^{2} \mathbf{I}_{N}\right][24]$. In this way, the detector does not need the information of the remaining users.

The equalized vector in this case is given by

$$
\mathbf{y}_{M M S E}=\mathbf{A}_{M M S E}^{m} \tilde{\mathbf{z}}_{m} .
$$

Subsequently, the components of the desired vector are detected by a minimum-distance receiver. 


\section{3 \\ Numerical Results: OFDMA and CS-CDMA in time-varying linear chan- nels}

In this section, the numerical results obtained by the OFDMA and CSCDMA systems in time-varying linear channels are analyzed. We consider that both systems have the same equivalent discrete channel impulse response and the same transmission bandwidth. The two systems transmit the same number of information symbols $(M=K N)$ and they have the same number of active users. The BER versus $E_{B} / N_{0}$ curves are used to evaluate the performance of the systems. In this case, the Energy per information bit transmitted $E_{B}$ is also used. The sections 3.1 and 3.2 provide the mathematical expression used to obtain the performance curves.

The channels associated to the $K$ active users are modeled with the Clarke model $[22,23]$. The Clarke model establishes a temporal correlation $\mathbb{E}\left[\alpha_{k, p}(i+\tau) \alpha_{k, p}(i)\right]=\sigma^{2} J_{0}\left(2 \pi f_{d} T_{s} \tau\right)$ to model the channel gains $\left(\alpha_{k, p} p=\right.$ $0,1, \ldots, P-1)$, where $J_{0}($.$) represents the Bessel function of first kind of order$ zero, $f_{d}$ is the Doppler frequency and $T_{s}=1 / R_{s}, R_{s}$ being the transmission rate. The Doppler frequency is considered the same for all channels. The components $\left\{\alpha_{k, i},\right\}_{i=0}^{P-1}$ are computed as explained in Section 3.2, where the values of the coefficients are correlated. During the simulations, the Sum of Sinusoids technique was a fast way to find the $\alpha_{k, i}$ components [25]. The weights are obtained using the relationship $p_{i}=10^{\frac{-i}{P-1}}$ with $i=0,1, . ., P-1$, which satisfies that $\sum_{i=0}^{P-1}\left|p_{i}\right|^{2}=1$.

The term $f_{d} T_{s}$ determines the amount of variation that the channel suffers. In this way, the propagation channels can be semi-static or with fast variations. The semi-static channel is when the channel components do not change within each transmitted block, that is when the channel variations are slow $\left(f_{d} T_{s}=10^{-6}\right.$ to $\left.10^{-4}\right)$. The fast variations occur when the channel components change with each symbol inside a block, that is when the channel variations are fast $\left(f_{d} T_{s}=10^{-3}, 10^{-2}\right)$.

Table 3.1 presents the parameters used to simulate the performance curves corresponding to random, multipath, linear and time-varying channel.

Figures 3.1, 3.2 and 3.3 illustrate the performance of the OFDMA and CS-CDMA systems in time-varying linear channels for different values of the term $f_{d} T_{s}$ in uplink configuration. Figure 3.1 shows the BER curves corresponding to the channel with slow variations $\left(f_{d} T_{s}=10^{-6}\right)$, whose results are quite similar to those illustrated in Figure 2.4 using the time-invariant channel in Section 2.3.

Figure 3.2 illustrates the systems performance in channels with $f_{d} T_{s}=$ 
Table 3.1: Simulation parameters - random, multipath, linear and time-varying channel.

\begin{tabular}{lcc}
\hline \hline Parameters & OFDMA & CS-CDMA \\
\hline \hline Modulation & QPSK & QPSK \\
Channel taps $(P)$ & 4 & 4 \\
Sub-carriers $(M)$ & 64,48 & - \\
Data vector length $(N)$ & - & 8,12 \\
Number of channel realizations & 50000 & 50000 \\
Number of information symbols sent & $32 \times 10^{5}$ & $32 \times 10^{5}$ \\
& $24 \times 10^{5}$ & $24 \times 10^{5}$ \\
Users $(K)$ & 8,4 & 8,4 \\
$f_{d} T_{s}$ & $10^{-6}, 10^{-4}, 10^{-3}$ & $10^{-6}, 10^{-4}, 10^{-3}$ \\
\hline
\end{tabular}

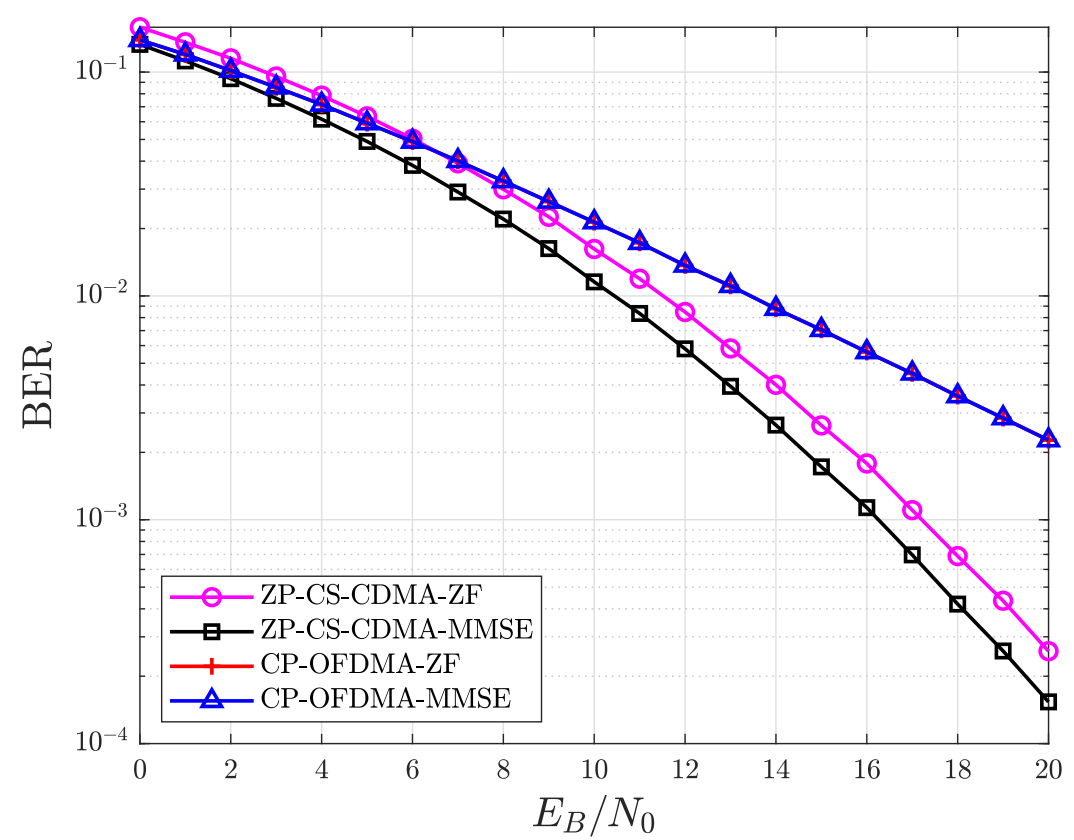

Figure 3.1: BER vs $E_{B} / N_{0}$ curves of OFDMA and CS-CDMA systems with a set of parameters $f_{d} T_{s}=10^{-6}, L=4, M=64, N=8, K=8, B=8$ and $P=4$, in uplink configuration.

$10^{-4}$, which are close to those shown in Figure 3.1 corresponding to $f_{d} T_{s}=$ $10^{-6}$. This fact indicates that the systems are relative robust, because they are able to maintain the performance despite slightly altering the channel conditions. On the other hand, Figure 3.3 shows the BER curves for $f_{d} T_{s}=$ $10^{-3}$, where fast variations are introduced in the channel. In this case, we can note that the MMSE performance in OFDMA had little change, but the ZF equalizer exhibits a worse performance. By analyzing the CS-CDMA system, we can observe that both equalizers, ZF and MMSE, suffer performance drops compared to the cases $f_{d} T_{s}=10^{-6}$ and $10^{-4}$, illustrated in Figures 3.1 and 
3.2 .

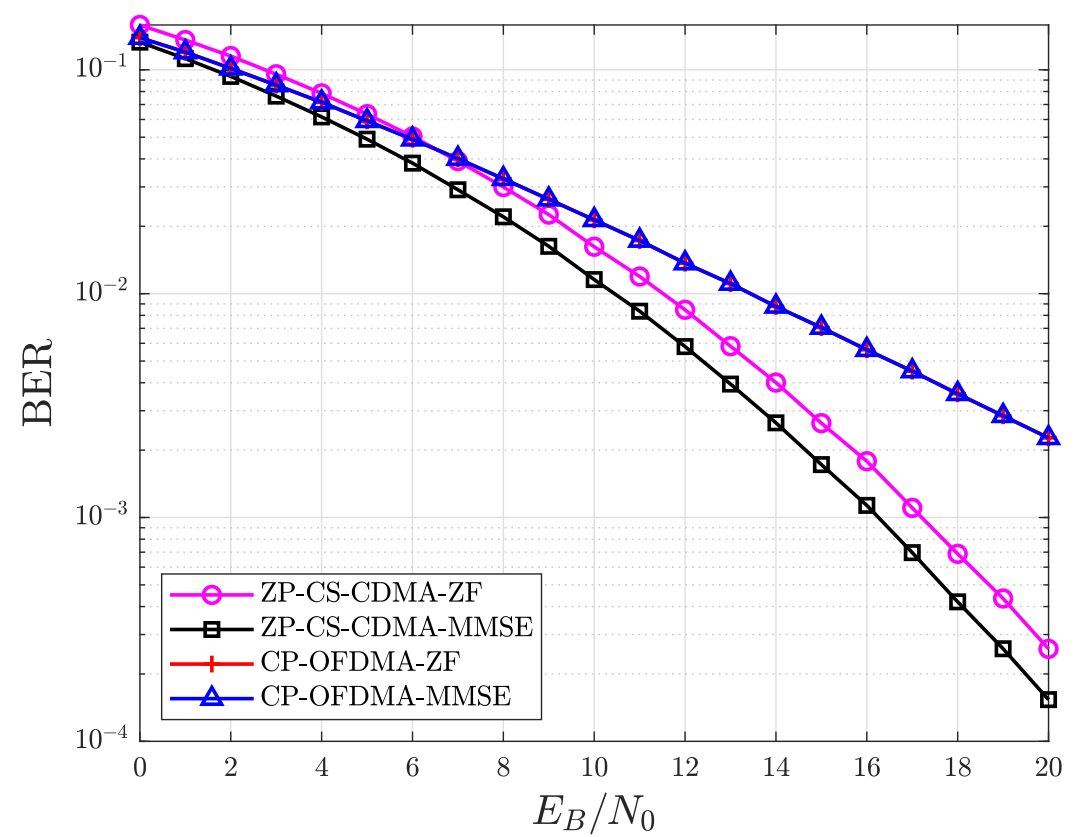

Figure 3.2: BER vs $E_{B} / N_{0}$ curves of OFDMA and CS-CDMA systems with a set of parameters $f_{d} T_{s}=10^{-4}, L=4, M=64, N=8, K=8, B=8$ and $P=4$, in uplink configuration.

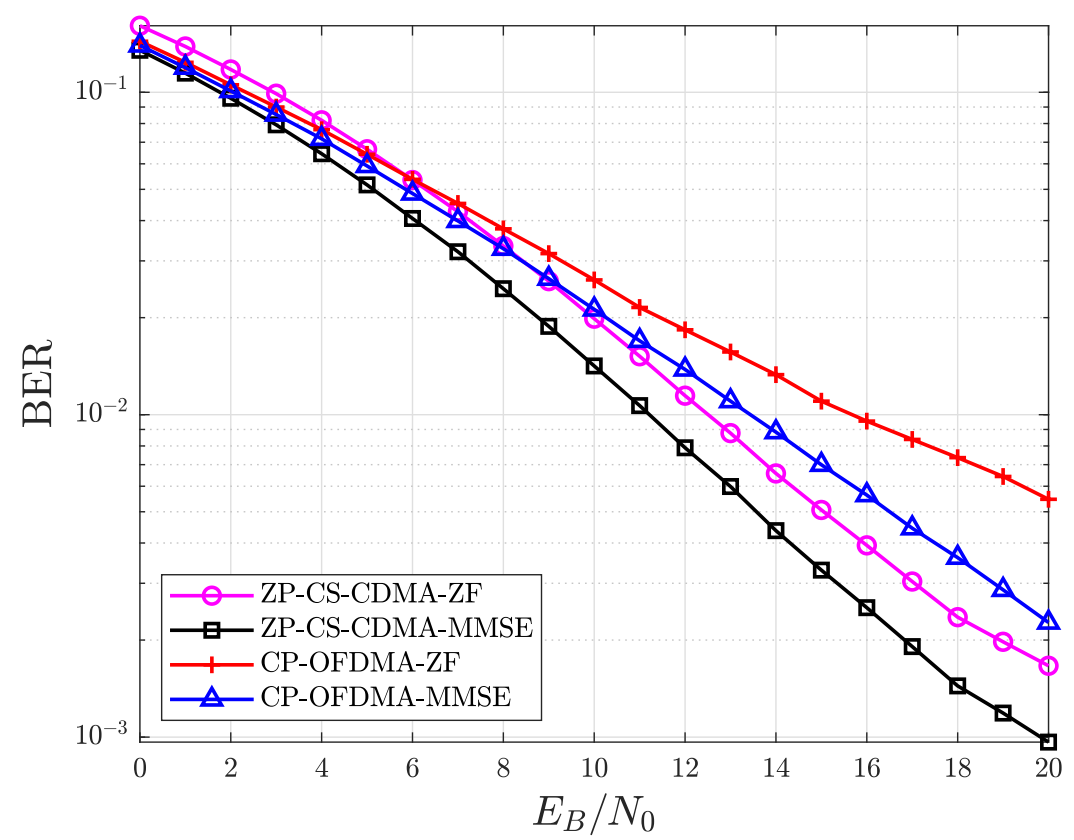

Figure 3.3: BER vs $E_{B} / N_{0}$ curves of OFDMA and CS-CDMA systems with a set of parameters $f_{d} T_{s}=10^{-3}, L=4, M=64, N=8, K=8, B=8$ and $P=4$, in uplink configuration. 
The performance curves obtained in the downlink configuration are shown in the figures 3.4, 3.5 and 3.6. The downlink and uplink results are quite similar since the performance curves are very close, which is an expected result during the simulations.

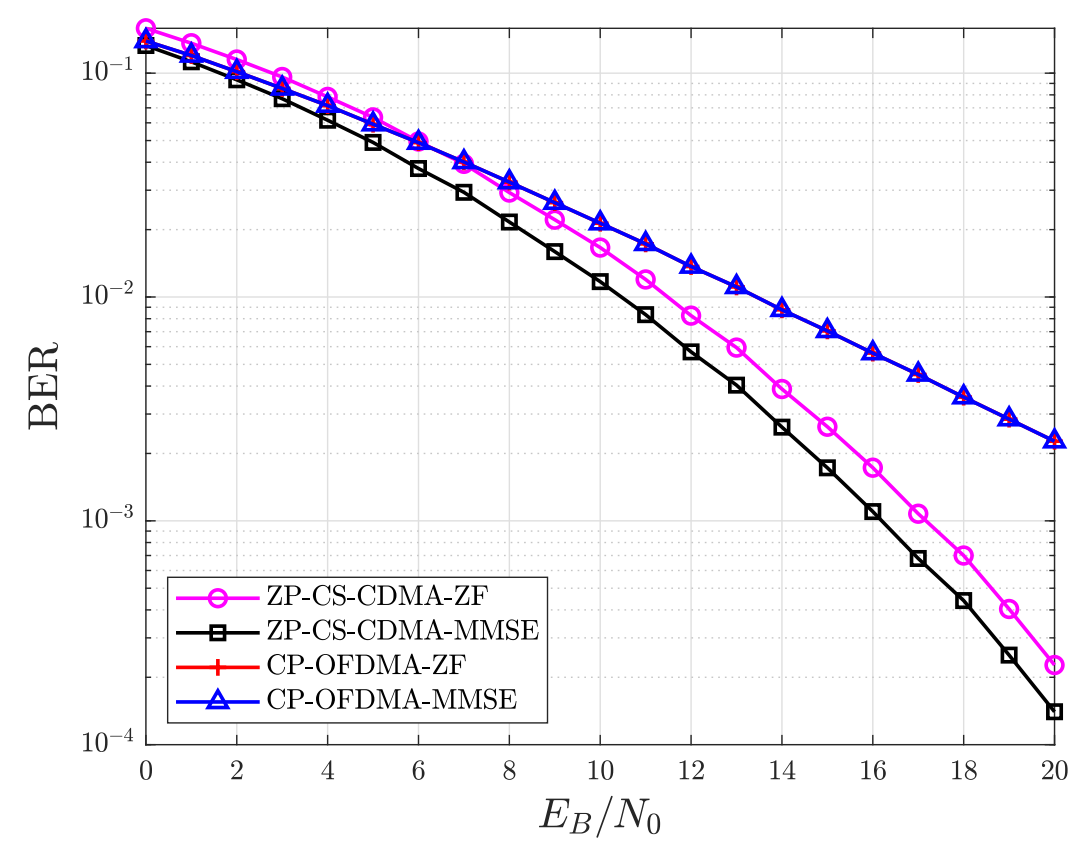

Figure 3.4: BER vs $E_{B} / N_{0}$ curves of OFDMA and CS-CDMA systems with a set of parameters $f_{d} T_{s}=10^{-6}, L=4, M=64, N=8, K=8, B=8$ and $P=4$, in downlink configuration.

In this section, the simulations of the downlink configuration were performed assuming a simultaneous detection for the OFDMA system while CS-CDMA does not. This fact brings advantages to OFDMA since it assumes that in the downlink configuration each user knows the information of the other users. However, CS-CDMA maintains better performance as shown in the figures $3.4,3.5$ and 3.6.

Figures 3.7, 3.8 and 3.9 illustrate the performance results for the second parameter set in the uplink configuration. Figure 3.7 shows the performance curves for $f_{d} T_{s}=10^{-6}$, which again matches the performance achieved by the time-invariant linear channel in Figure 2.9. This behavior is caused by the slow variations that the channel suffers. In other words, the channel is maintained approximately constant during a symbol interval.

Figure 3.8 corresponds to $f_{d} T_{s}=10^{-4}$ and the performance curves show that the systems are not affected by the variations introduced. On the other hand, Figure 3.9 illustrates the results for $f_{d} T_{s}=10^{-3}$, where a performance drop in the CS-CDMA system is evidenced. For OFDMA, the MMSE equalizer 


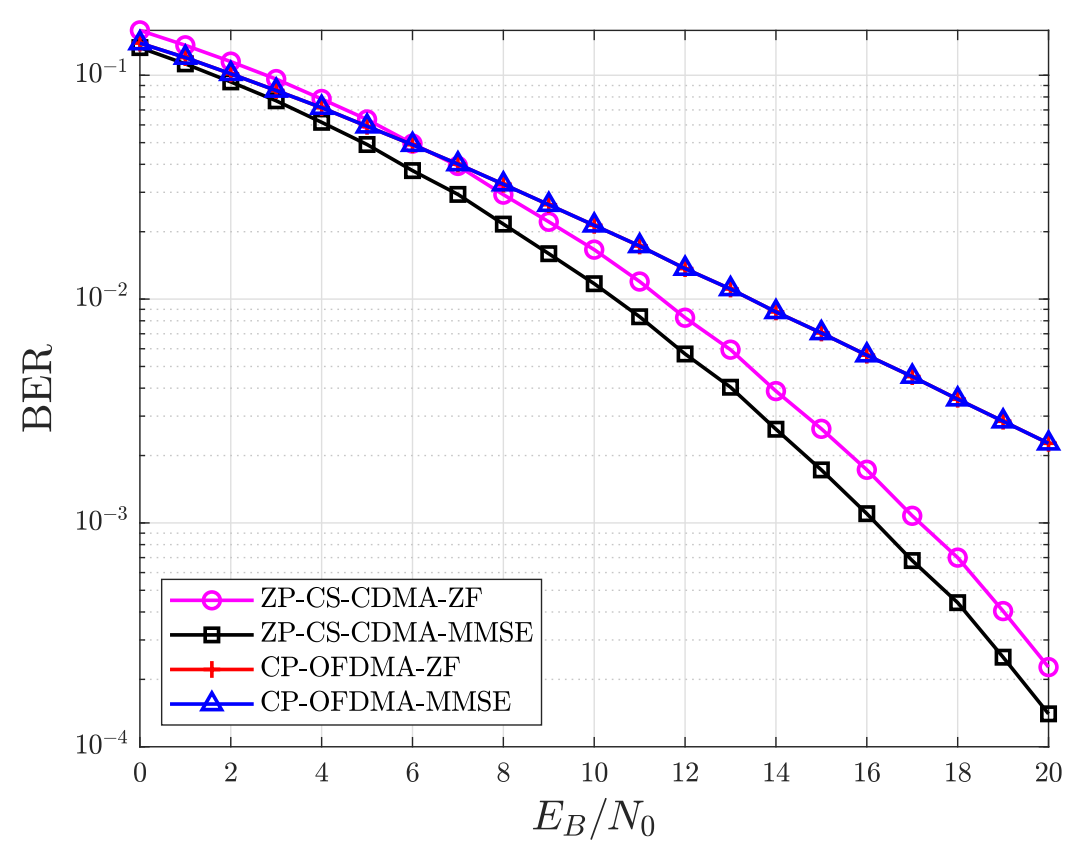

Figure 3.5: BER vs $E_{B} / N_{0}$ curves of OFDMA and CS-CDMA systems with a set of parameters $f_{d} T_{s}=10^{-4}, L=4, M=64, N=8, K=8, B=8$ and $P=4$, in downlink configuration.

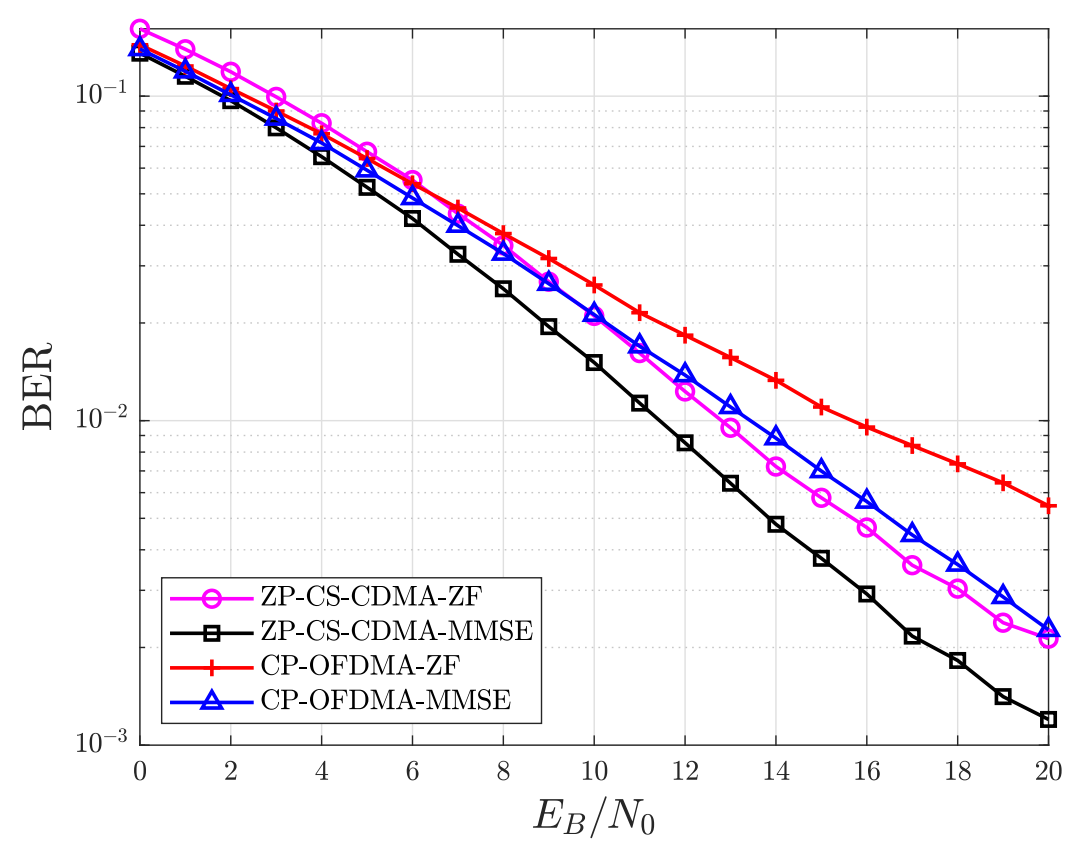

Figure 3.6: BER vs $E_{B} / N_{0}$ curves of OFDMA and CS-CDMA systems with a set of parameters $f_{d} T_{s}=10^{-3}, L=4, M=64, N=8, K=8, B=8$ and $P=4$, in downlink configuration. 


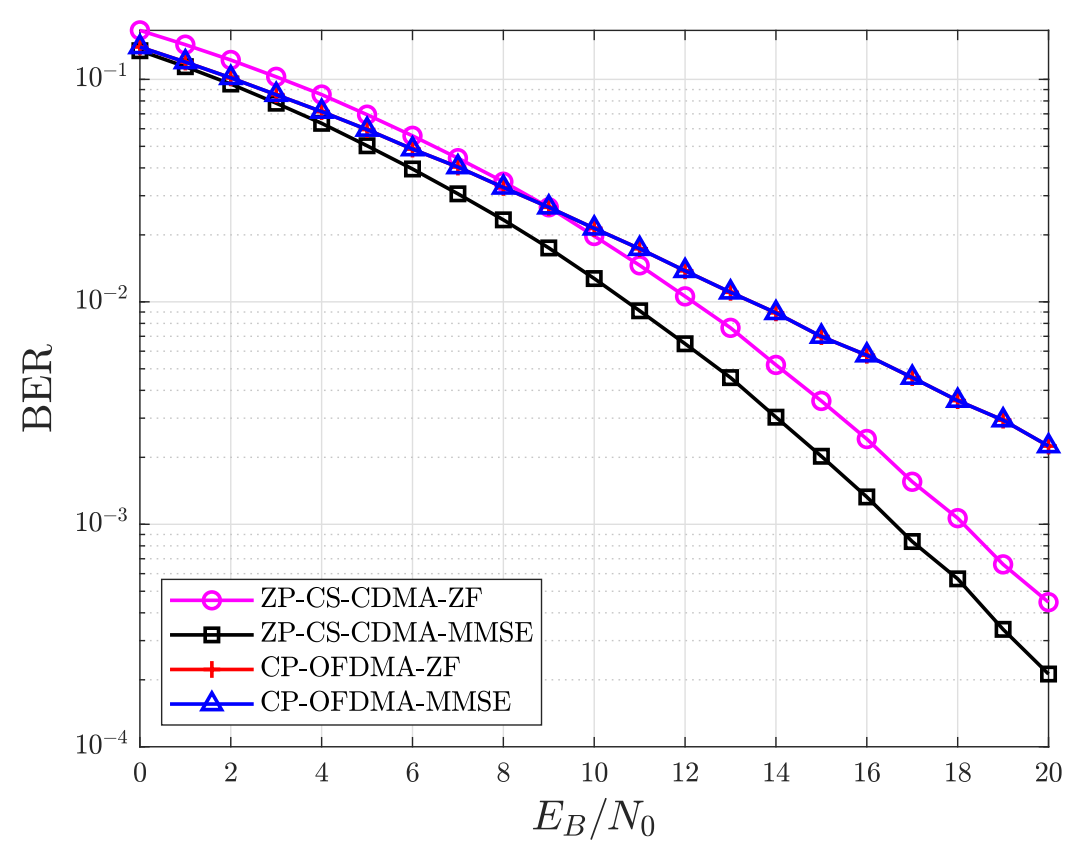

Figure 3.7: BER vs $E_{B} / N_{0}$ curves of OFDMA and CS-CDMA systems with a set of parameters $f_{d} T_{s}=10^{-6}, L=4, M=48, N=12, K=4, B=12$ and $P=4$, in uplink configuration.

is almost unaffected by the fast variations introduced in the channel while the ZF equalizer of the OFDMA system suffers a performance drop. We can note that the CS-CDMA system was more affected in both cases when the timeinvariant characteristics of the channel change. The OFDMA system was more robust against the fast variations in the channel $\left(f_{d} T_{s}=10^{-3}\right)$. 


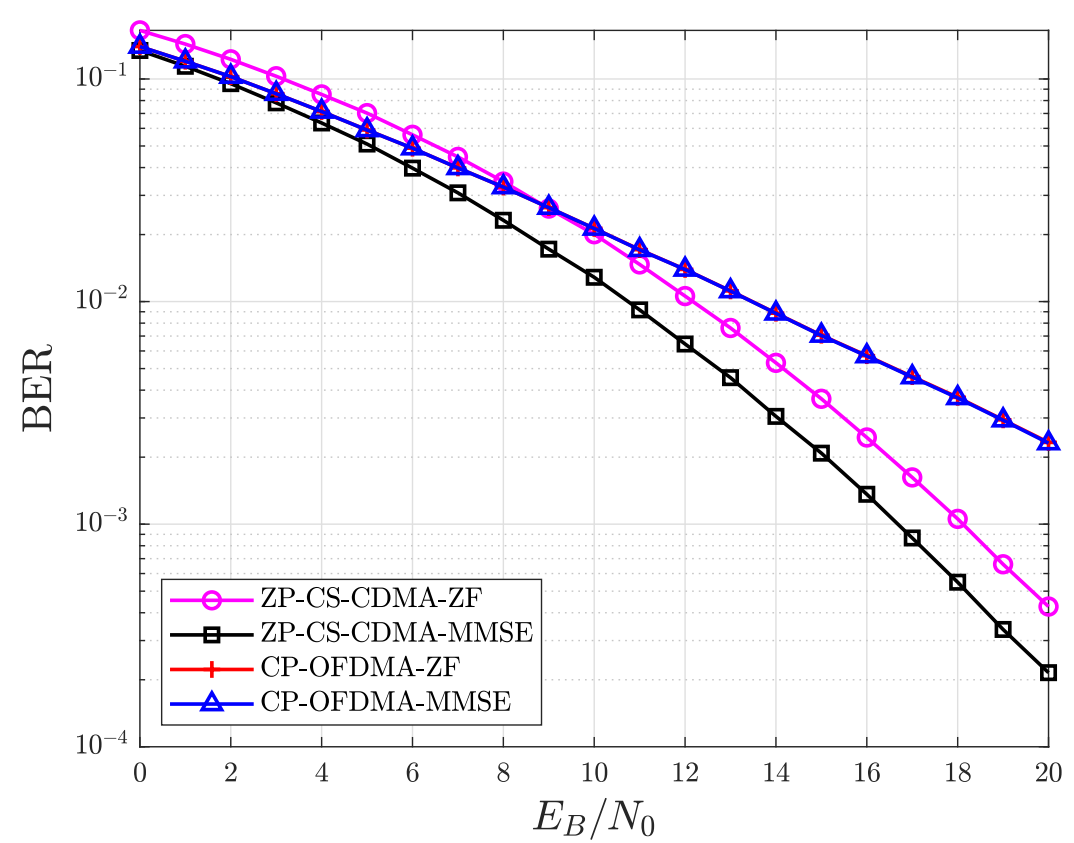

Figure 3.8: BER vs $E_{B} / N_{0}$ curves of OFDMA and CS-CDMA systems with a set of parameters $f_{d} T_{s}=10^{-4}, L=4, M=48, N=12, K=4, B=12$ and $P=4$, in uplink configuration.

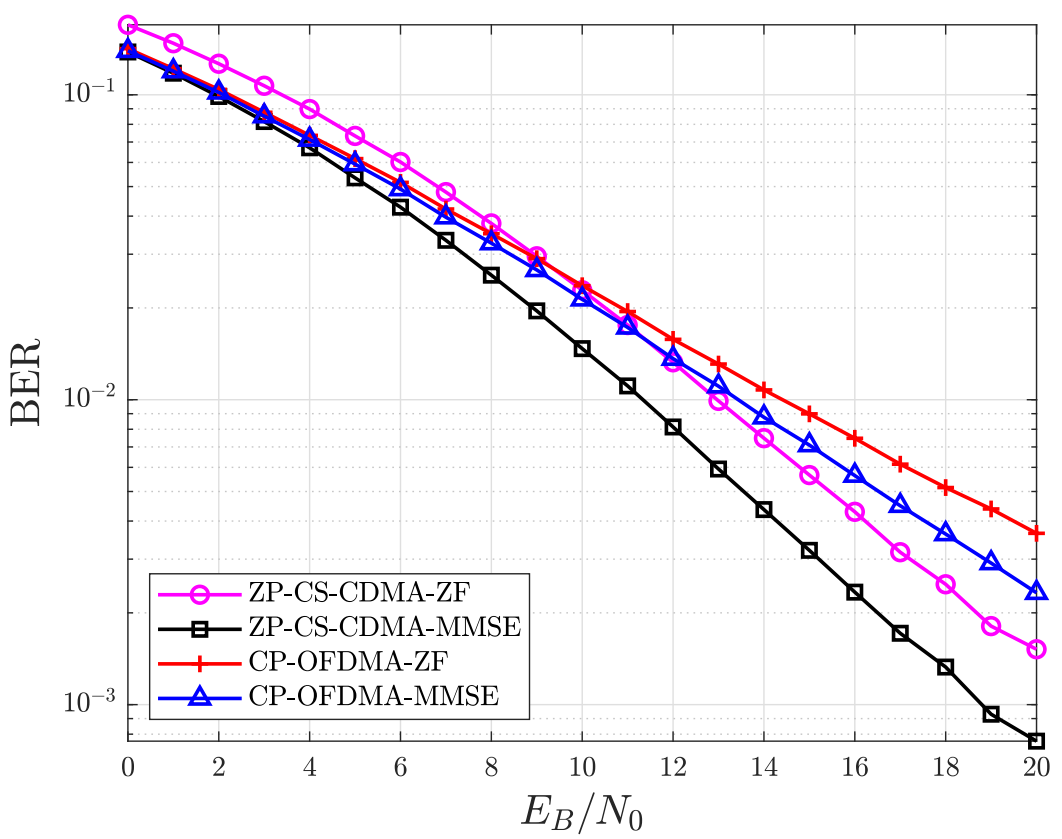

Figure 3.9: BER vs $E_{B} / N_{0}$ curves of OFDMA and CS-CDMA systems with a set of parameters $f_{d} T_{s}=10^{-3}, L=4, M=48, N=12, K=4, B=12$ and $P=4$, in uplink configuration. 


\section{4 \\ Block Transmission Schemes in time-invariant non-linear channels}

In this chapter, the transmission is carried out in the presence of a Power Amplifier (PA). This device introduces non-linearities in the transmission signal. The amplification of the input signal depends on the position relative to the saturation point. The system achieves its best performance when operating in the linear region closest to the saturation point. Several concepts around PA are explained in this chapter. Finally, the behavior of the OFDMA and CS-CDMA systems in time-invariant non-linear channels is analyzed.

\section{1}

\section{OFDMA in non-linear channels}

OFDMA systems are seriously affected by non-linear channels due to Peak-to-Average Power Ratio (PAPR). This degradation is common in multicarrier systems. In this section, the mathematical model of OFDMA in nonlinear channels is covered. The formulations in Section 2.1.1 are used to develop the proposed model.

The transmitted vector $\mathbf{z}$ is the result of the multiplication of the data symbols vector and the IDFT matrix, such that

$$
\mathbf{z}=\left[\begin{array}{llll}
z_{1} & z_{2} & \ldots & z_{M}
\end{array}\right]^{T}=\mathbf{W}^{H} \mathbf{d},
$$

where we consider that $\mathbb{E}\left[\left|d_{i}\right|^{2}\right]=1$.

The non-linear transformation caused by the influence of PA is illustrated in Figure 4.1.

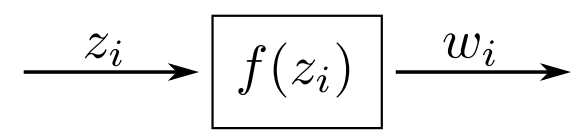

Figure 4.1: Non-linear transformation scheme.

The non-linear polynomial transformation is assumed to model the effects introduced by the PA $[26,27]$, such that

$$
\tilde{w}_{i}=\sum_{l=0}^{L^{\prime}} b_{l} z^{l},
$$


which can be expressed in terms of complex envelopes [28] as

$$
w_{i}=\sum_{m=0}^{M^{\prime}} \gamma_{2 m+1} z_{i}^{m+1} z_{i}^{* m}
$$

where

$$
\gamma_{2 m+1}=\frac{b_{2 m+1}}{2^{2 m}}\left(\begin{array}{c}
2 m+1 \\
m
\end{array}\right)
$$

and $M^{\prime}=\left\lfloor\left(L^{\prime}-1\right) / 2\right\rfloor$, meaning that $m$ is the largest integer less than or equal to $\left(L^{\prime}-1\right) / 2$. This non-linearity could represent, for example, a transmitter PA operating in its non-linear region. The expression (4-3) can be rewritten as

$$
w_{i}=z_{i} \sum_{m=0}^{M^{\prime}} \gamma_{2 m+1}\left|z_{i}\right|^{2 m},
$$

where using (4-4) and the known values $b_{0}=0, b_{1}=1, b_{2}=0$ and $b_{3}=-1 / 4$ $[26,27]$, the corresponding $\gamma$ values can be determined. By substituting the $\left\{b_{1}, b_{2}, b_{3}, b_{4}\right\}$ values in (4-4), we have that

$$
\begin{gathered}
\text { for } m=0 \longrightarrow \gamma_{1}=\frac{b_{1}}{2^{0}}\left(\begin{array}{l}
1 \\
0
\end{array}\right)=1 \\
\text { for } m=1 \longrightarrow \gamma_{3}=\frac{b_{3}}{2^{2}}\left(\begin{array}{l}
3 \\
1
\end{array}\right)=-\frac{3}{16} .
\end{gathered}
$$

The computed $\gamma$ values are substituted in (4-5) for obtaining the components of the new transmitted vector resulting from non-linearity, such that

$$
w_{i}=z_{i}\left(1+\gamma_{3}\left|z_{i}\right|^{2}\right)
$$

The non-linear transformation expressed in (4-6) only modifies the amplitude, the phase is maintained. For this reason, the module operation is applied to (4-6), such that

$$
\left|w_{i}\right|=\left.\left|z_{i}\right|\left|1+\gamma_{3}\right| z_{i}\right|^{2} \mid
$$

where the relationship between $\left|w_{i}\right|$ and $\left|z_{i}\right|$ could be represented as $\left|w_{i}\right|=$ $f\left(\left|z_{i}\right|\right)$. It is assumed here that the output of the non-linear device remains constant from its maximum value. This modification introduces the concept of saturation region. The expression (4-7) is then rewritten to match the PA behavior,

$$
\left|w_{i}\right|= \begin{cases}\left|z_{i}\right|+\gamma_{3}\left|z_{i}\right|^{3}, & \left|z_{i}\right|<a \\ b, & \left|z_{i}\right| \geq a\end{cases}
$$

where $a=4 / 3$ and $b=8 / 9$ are the coordinates of the maximum point of the initial expression of $w_{i}(4-7)$. Another detail is the one referring to the second part in (4-8), when a power analysis is performed, the terms are squared and only $b^{2}$ remains, due to $\left|\frac{z_{i}}{\left|z_{i}\right|}\right|^{2}=1$. The graphic corresponding to (4-8) is illustrated in Figure 4.2. 


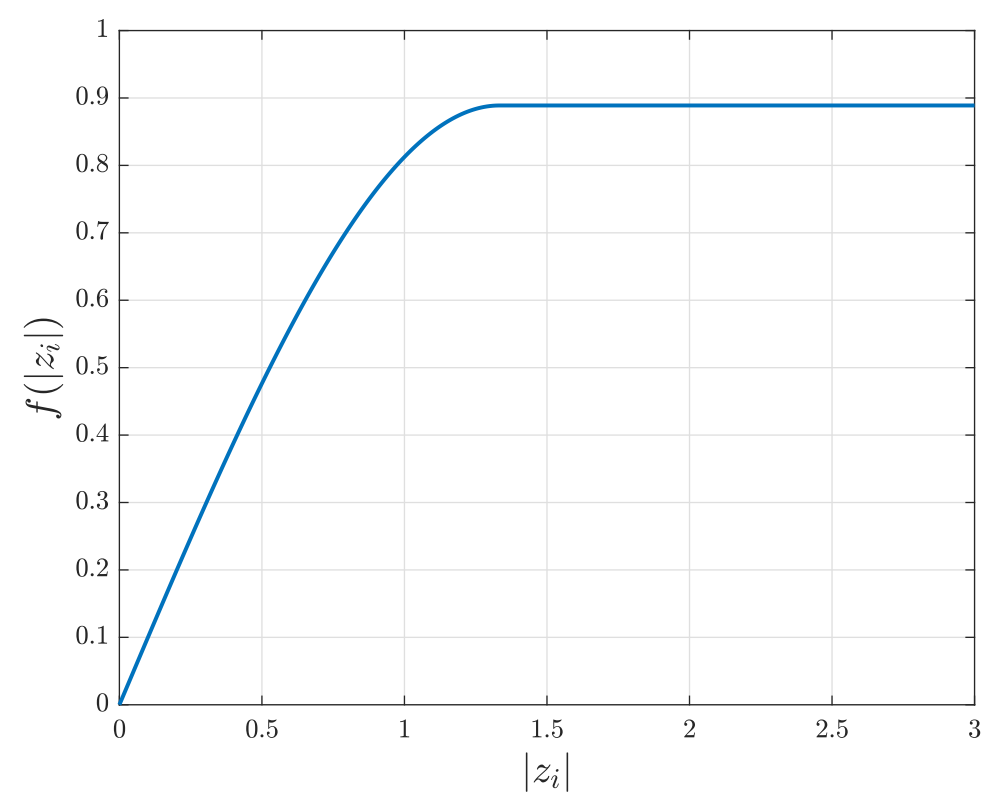

Figure 4.2: Graphic representation of $\left|w_{i}\right|=f\left(\left|z_{i}\right|\right)$.

In Figure 4.2 for $\left|z_{i}\right|>a$, the system enters the saturation zone, since any value of $\left|z_{i}\right|$ greater than $a$ has the same amplification, $b$.

In non-linear systems there are several figures of merit that characterize the system behavior. The input power $\left(P_{i n}\right)$ or input energy is the quadratic mean of the input signal $\alpha z_{i}$, such that

$$
P_{\text {in }}=\alpha^{2} \mathbb{E}\left[\left|z_{i}\right|^{2}\right]=\alpha^{2}
$$

where $\alpha$ is a power adjusting factor.

The input Back-off (IBO) is the ratio between the input power and the saturation input power $\left(P_{\text {sat_in }}\right)$ :

$$
\mathrm{IBO}=\frac{P_{\text {in }}}{P_{\text {sat_in }}},
$$

with the input power given by (4-9). The saturation input power is $a^{2}$, which is extracted from Figure 4.2 and the input Back-off is a system parameter. Then,

and

$$
\mathrm{IBO}=\frac{\alpha^{2}}{a^{2}},
$$

$$
\alpha=\sqrt{\operatorname{IBO}} a .
$$

The output power depends on the selected input Back-off. Here the power values can be replaced by energy. The power is an unit that represents energy per time. The output energy is given by 


$$
E_{\text {out }}=\mathbb{E}\left[\left|w_{i}\right|^{2}\right]= \begin{cases}\mathbb{E}\left[\left(\alpha\left|z_{i}\right|+\gamma_{3} \alpha^{3}\left|z_{i}\right|^{3}\right)^{2}\right], & \alpha\left|z_{i}\right|<a \\ b^{2}, & \alpha\left|z_{i}\right| \geq a\end{cases}
$$

where the conditions depend on the adjust term alpha, which was computed in (4-12). In this way, the condition in (4-8) is represented by

$$
\begin{array}{r}
\alpha\left|z_{i}\right|=\sqrt{\operatorname{IBO}} a\left|z_{i}\right|<a, \text { or } \\
\left|z_{i}\right|^{2}<1 / \text { IBO } .
\end{array}
$$

Once the output energy is computed, the Output Back-off (OBO) can be found with the relation given by

$$
\mathrm{OBO}=\frac{P_{\text {out }}}{P_{\text {sat_out }}}=\frac{E_{\text {out }}}{b^{2}},
$$

where $P_{\text {out }}$ is the output power, $P_{\text {sat_out }}$ is the saturation output power and $E_{\text {out }}$ is the output energy.

In OFDMA, the signal received in the uplink configuration is expressed by (2-9), but due to the action of non-linearity, it is necessary to rewrite the equation as

$$
\begin{aligned}
\mathbf{r} & =\operatorname{Diag}(\mathbf{q}) \mathbf{d}+\mathbf{n} \\
& =\operatorname{Diag}(\mathbf{q}) \mathbf{W}_{M} \mathbf{W}_{M}^{H} \mathbf{d}+\mathbf{n} \\
& =\operatorname{Diag}(\mathbf{q}) \mathbf{W}_{M} \mathbf{w}+\mathbf{n}
\end{aligned}
$$

where $\mathbf{w}=\left[\begin{array}{c}w_{1} \\ \vdots \\ w_{M}\end{array}\right]$ and are generated by

$$
w_{i}= \begin{cases}\alpha z_{i}\left(1+\gamma_{3} \alpha^{2}\left|z_{i}\right|^{2}\right), & \left|z_{i}\right|^{2}<1 / \text { IBO } \\ b \frac{z_{i}}{\left|z_{i}\right|}, & \left|z_{i}\right|^{2} \geq 1 / \text { IBO }\end{cases}
$$

where $w_{i}$ is the result of the non-linearity of the channel.

Each component $w_{i}$ of the vector $\mathbf{w}$ is normalized by the output energy as

$$
w_{i}=\sqrt{E_{\text {out }}} \frac{w_{i}}{\sqrt{E_{\text {out }}}}=\sqrt{E_{\text {out }}} \tilde{w}_{i},
$$

where $\tilde{w}_{i}$ is the resulting normalized component. The noise components are also normalized with respect to the noise standard deviation

$$
n_{i}=\sigma \frac{n_{i}}{\sigma}=\sigma \tilde{n}_{i}
$$

where $\tilde{n}_{i}$ is the normalized noise component. Substituting (4-18) and (4-19) in 
(4-16), we obtain

$$
\mathbf{r}=\sqrt{E_{\text {out }}} \operatorname{Diag}(\mathbf{q}) \mathbf{W}_{M} \tilde{\mathbf{w}}+\sigma \tilde{\mathbf{n}} .
$$

Applying (4-15) to (4-20), we have that

$$
\begin{aligned}
\mathbf{r} & =\sqrt{E_{\text {sat }} \mathrm{OBO}} \operatorname{Diag}(\mathbf{q}) \mathbf{W}_{M} \tilde{\mathbf{w}}+\sigma \tilde{\mathbf{n}} \\
& =\sigma\left(\sqrt{\left.\left(\frac{E_{\text {sat }}}{\sigma^{2}}\right) \text { OBO } \operatorname{Diag}(\mathbf{q}) \mathbf{W}_{M} \tilde{\mathbf{w}}+\tilde{\mathbf{n}}\right)}\right.
\end{aligned}
$$

where $E_{\text {sat }}$ is the output saturation energy.

In this case, OFDMA uses the cyclic prefix as guard interval. Therefore, to obtain the noise-to-signal ratio per information symbol characterized by $E_{\text {sat }}^{s} / \sigma^{2}$, it is necessary to include the factor $M /(M+L)$, since the system sends $M+L$ data symbols but only $M$ data symbols are information symbols. Applying this to (4-21),

$$
\mathbf{r}=\sigma\left(\sqrt{\left(\frac{E_{\text {sat }}^{s}}{\sigma^{2}}\right)\left(\frac{M}{M+L}\right) \text { OBO }} \operatorname{Diag}(\mathbf{q}) \mathbf{W}_{M} \tilde{\mathbf{w}}+\tilde{\mathbf{n}}\right)
$$

The $m$-th user only detects the symbols intended to him as explained in Section 2.1.1.2.

\subsection{1}

\section{OFDMA Equalization Strategies}

The linear detectors based in Zero-Forcing (ZF) eliminate the interference by filtering the received signal with an inverse of the channel matrix. This equalizer does not consider the effects of noise [18, 19], and therefore the noise may be enhanced in the process of eliminating the interference. The propagation is carried out through a time-invariant non-linear channel, hence the channel characteristics are maintained during the transmission of an OFDMA block as explained in Section 2.1.2. The expression (2-22) represents the ZF equalizer.

In the case of Minimum Mean Square Error (MMSE), the equalizer offers improvements over the ZF equalization, especially in relation to the poor performance in low SNR regimes of the ZF. The MMSE equalizer achieves a trade-off between the noise level and interference at the receiver, by maximizing the sum of the signal-to-noise ratio and interference [29]. In this case, the MMSE equalizer is analyzed in time-invariant channels, therefore, the channel matrix is characterized by a diagonal matrix and the expression (2-23) represents the matrix of the MMSE equalizer. After applying the corresponding equalizer, a minimum distance receiver performs the detection of symbols. 


\section{2}

\section{CS-CDMA in non-linear channels}

CS-CDMA systems are also affected when the transmission is through non-linear channels. In this section, the signal model is analyzed and it is supported by the equations in Section 2.2.1. The corresponding transmitter vector, $\mathbf{z}_{i}$, is formed by size $N$ blocks of data symbols spreading the chips of the user code sequence. Thus, the $N \times 1$ transmitter vector can be expressed as

$$
\mathbf{z}_{i}=\sum_{k=1}^{K} \mathbf{d}_{k} c_{k, i}, \quad i=1,2, \ldots, B
$$

where $c_{k, i}$ is the chip corresponding to the sequence of the $k$-th user and it is assumed that $\mathbb{E}\left[\left|d_{i}\right|^{2}\right]=1$.

The same non-linear channel model employed in OFDMA (Section 4.1), illustrated in Figure 4.1 and modeled by equations (4-2)-(4-8) is used in the analysis. The saturation zone in this case is also the same and the behavior of $\left|w_{i}\right|$ is characterized in Figure 4.2.

In CS-CDMA an appropriate metric to measure the system behavior in non-linear channels is the input power, which is given by

$$
\begin{aligned}
P_{\text {in }} & =\alpha^{2} \mathbb{E}\left[\left|z_{i}\right|^{2}\right]=\alpha^{2} \frac{N}{N+L} \\
& =\alpha^{2} p
\end{aligned}
$$

where $\alpha$ is an adjust term and $p=\frac{N}{N+L}$. The new term $p$ is a consequence of zeros at the end of the vector, due to the use Zero Padding as guard interval.

The input Back-off given by (4-10) is then

$$
\text { IBO }=\frac{\alpha^{2} p}{a^{2}}
$$

and therefore

$$
\alpha=\sqrt{\frac{\mathrm{IBO}}{p}} a .
$$

With these results, the output energy can be computed using an expression similar to (4-13), but applying a small change in the conditions terms. The new conditions are given by

$$
\begin{array}{r}
\sqrt{\frac{\text { IBO }}{p}} a\left|z_{i}\right|<a \text { or } \\
\left|z_{i}\right|^{2}<p / \text { IBO }
\end{array}
$$

The output energy for the CS-CDMA system is obtained by substituting (4-27) in (4-13), such that 


$$
E_{\text {out }}=\mathbb{E}\left[\left|w_{i}\right|^{2}\right]= \begin{cases}\mathbb{E}\left[\left(\alpha\left|z_{i}\right|+\gamma_{3} \alpha^{3}\left|z_{i}\right|^{3}\right)^{2}\right], & \left|z_{i}\right|^{2}<p / \text { IBO } \\ b^{2}, & \left|z_{i}\right|^{2} \geq p / \text { IBO }\end{cases}
$$

The signal received for the CS-CDMA system in linear and time-invariant channels was obtained in (2-28), assuming the guard interval ZP. In this case, the transmission is carried out through a time-invariant non-linear channel, which introduces modifications in (2-28). The downlink configuration is assumed, therefore, considering the signal received by user $m\left(\overline{\mathbf{H}}_{k}^{(\mathbf{0})}=\right.$ $\overline{\mathbf{H}}_{m}^{(\mathbf{0})}$, all $k$ ), the expression (2-28) can be rewritten as

$$
\mathbf{r}_{Z P}^{(m)}(i)=\overline{\mathbf{H}}_{m}^{(\mathbf{0})} \mathbf{z}_{i}+\mathbf{n}(i) \quad i=0,1, \ldots, B-1
$$

where $\mathbf{z}_{i}$ is computed in (4-23). By collecting $B$ consecutive received signals, a $(N+L) \times B$ observation matrix is formed, such that

$$
\mathbf{R}_{m}=\left[\mathbf{r}_{m}(0) \ldots \mathbf{r}_{m}(B-1)\right]=\overline{\mathbf{H}}_{m}^{(\mathbf{0})} \mathbf{Z}+\mathbf{N},
$$

where $\mathbf{Z}=\sum_{k=1}^{K} \mathbf{d}_{k} \mathbf{c}_{k}^{T}$ and $\mathbf{N}=[\mathbf{n}(0) \ldots \mathbf{n}(B-1)]$. Applying the non-linear transformation represented in Figure 4.1, we obtain

$$
\mathbf{R}_{m}=\left[\mathbf{r}_{m}(0) \ldots \mathbf{r}_{m}(B-1)\right]=\overline{\mathbf{H}}_{m}^{(\mathbf{0})} \mathbf{P}+\mathbf{N},
$$

where the $N \times B$ matrix $\mathbf{P}$ is the non-linear transformation of the $N \times B$ matrix $\mathbf{Z}$, such that

$$
\mathbf{P}=f(\mathbf{Z})=\left[\begin{array}{lll}
\mathbf{w}_{1}^{\prime} & \ldots & \mathbf{w}^{\prime}
\end{array}\right],
$$

where $\mathbf{w}^{\prime}{ }_{b}=\left[w_{b, 1} \ldots w_{b, N}\right]^{T}$ with $b=1, . ., B$ and represents the column vectors of the matrix $\mathbf{P}$. The components $w_{b, i}$ with $i=1, . ., N$ are computed as

$$
w_{b, i}= \begin{cases}\alpha z_{b, i}\left(1+\gamma_{3} \alpha^{2}\left|z_{b, i}\right|^{2}\right), & \left|z_{b, i}\right|^{2}<p / \text { IBO } \\ b \frac{z_{b, i}}{\left|z_{b, i}\right|}, & \left|z_{b, i}\right|^{2} \geq p / \text { IBO }\end{cases}
$$

where $z_{b, i}$ are the components of $\mathbf{Z}$. Each element $w_{b, i}$ of the matrix $\mathbf{P}$ is normalized as

$$
\tilde{w}_{b, i}=\frac{w_{b, i}}{\sqrt{E_{\text {out }} / p}}
$$

since the inverse of $p$ allows removing the contribution of zeros in the output energy computed in (4-28). Now, $\tilde{w}_{b, i}$ are the components of the normalized matrix $\tilde{\mathbf{P}}$. The noise components $n_{b, i}$ are also normalized in relation to the standard deviation, such that

$$
\tilde{n}_{b, i}=\frac{n_{b, i}}{\sigma},
$$

where $\tilde{n}_{b, i}$ are the components of the normalized matrix $\tilde{\mathbf{N}}$. Applying (4-34) and (4-35) in (4-31), we have that 


$$
\mathbf{R}_{m}=\sqrt{E_{\text {out }}} \overline{\mathbf{H}}_{m}^{(\mathbf{0})} \tilde{\mathbf{P}}+\sigma \tilde{\mathbf{N}}
$$

and using (4-15), the previous expression can be rewritten as

$$
\begin{aligned}
\mathbf{R}_{m} & =\sqrt{E_{\text {sat_out } \mathrm{OBO}^{\prime}}} \overline{\mathbf{H}}_{m}^{(\mathbf{0})} \tilde{\mathbf{P}}+\sigma \tilde{\mathbf{N}} \\
& =\sigma\left(\sqrt{\left(\frac{E_{\text {sat_out }}}{\sigma^{2}}\right) \mathrm{OBO}^{\prime}} \overline{\mathbf{H}}_{m}^{(\mathbf{0})} \tilde{\mathbf{P}}+\tilde{\mathbf{N}}\right),
\end{aligned}
$$

where $\mathrm{OBO}^{\prime}=\mathrm{OBO} / p$, which is the Output Back-off without the contribution of the zeros. Note that in (4-34) the same division by $p$ was done. The next step towards detection is the product of the matrix $\mathbf{R}_{m}$ in (4-37) by the user $m$ code $\mathbf{c}_{m}$ to generate the signal vector

$$
\mathbf{r}_{m}=\mathbf{R}_{m} \mathbf{c}_{m}
$$

\subsection{1}

\section{CS-CDMA Equalization Strategies}

CS-CDMA systems analyzed in this chapter transmit through timeinvariant non-linear channels. In other words, the channel is maintained approximately constant during the transmission of a symbol block. This reason allows to use the same equations explained in Section 2.2.2. In this analysis, we emphasize the use of ZP as guard interval in the downlink configuration.

ZF and MMSE are two linear detectors widely used in transmission systems. These equalizers are represented by the corresponding matrices, such that

$$
\begin{gathered}
\overline{\mathbf{A}}_{Z F}^{(m)}=\mathbf{H}_{m}^{(0) \dagger} \text { and } \\
\overline{\mathbf{A}}_{M M S E}^{(m)}=\mathbf{H}_{m}^{(0) H}\left[\mathbf{H}_{m}^{(0)} \mathbf{H}_{m}^{(0) H}+\sigma^{2} \mathbf{I}\right]^{-1}
\end{gathered}
$$

where $(.)^{\dagger}$ corresponds to the pseudo-inverse operation. Once equalizers are applied to the signal $\mathbf{r}_{m}$ in (4-38), the detection of the equalized vector is performed using a component-wise minimum distance receiver.

\section{3}

\section{Numerical Results: OFDMA and CS-CDMA in time-invariant non-linear channels}

For comparison purposes, we note that the two systems have the same equivalent discrete channel impulse response and the same bandwidth. The number of information symbols transmitted by both systems are equal ( $M=$ $K N$ ) and they have the same number of active users. In this section, the performance curves are obtained when the signals propagate through time-invariant 
non-linear channels. The BER versus $E_{s a t}^{b}$ curves are used to evaluate the performance of the systems, where $E_{\text {sat }}^{b}$ represents the output saturation energy per bit transmitted. In non-linear systems, these curves are parameterized by the $\mathrm{OBO}$ since this value indicates the relationship between the output power and output saturation power as explained in Section 4.1.

In this work, the non-linear transformation is modeled as an intermediate block between the transmitter and the channel. Figure 4.1 shows the functionality of the intermediate block mentioned above. Sections 4.1 and 4.2 contain the expressions that were used to obtain the performance curves. The vector transmitted $\mathbf{w}$ resulting from the non-linear transformation is propagated through a time-invariant channel similar to the one used in Section 2.3. Therefore, the detection process can be realized by the linear equalizers ZF and MMSE.

The IBO is a system parameter, which is used to obtain $\alpha$ in (4-12). However, the performance curves were parameterized by the OBO to obtain a comparable signal-to-noise ratio at the output. In this case only are analyzed the non-linear effects without the amplification. The expected values required for the computation of $E_{\text {out }}$ in (4-13) and (4-28) were estimated empirically. An interpolation was used to find the $\mathrm{OBO}$ values corresponding to the initial IBO values. QPSK modulation was assumed in all scenarios considered in this section.

Figure 4.3 illustrates the performance curves for an OFDMA system in a time-invariant non-linear channel without the factor provided by the OBO in (4-22). In this case, the performance curves were carried out for different OBO values $(3 \mathrm{~dB}, 8 \mathrm{~dB}, 16 \mathrm{~dB}$ and $20 \mathrm{~dB})$. For high $\mathrm{OBO}$ values, the system performance is closer to the operation of the linear system. Figure 4.4 shows the performance curves of the same system, but using the MMSE equalizer. Both figures illustrate the same curves since the channel is time-invariant. Therefore, the channel matrix is a diagonal matrix as expressed in (4-22).

In figures 4.3 and 4.4 almost all curves coincide, except for $\mathrm{OBO}=3 \mathrm{~dB}$. The OFDMA-invariant curve corresponds to the system shown in Figure 2.6, where the channel is linear and time-invariant. These facts demonstrate that for higher values of $\mathrm{OBO}$ the system performance is closer to the operation of the linear system.

For CS-CDMA systems, the analysis in non-linear channels is the same. The behavior of the performance curves in relation to the $\mathrm{OBO}$ is similar to the OFDMA system. Figures 4.5 and 4.6 illustrate the performance curves of a CS-CDMA system using ZF and MMSE equalization respectively. In this case, the CS-CDMA-invariant curves also represent the results of the system in 


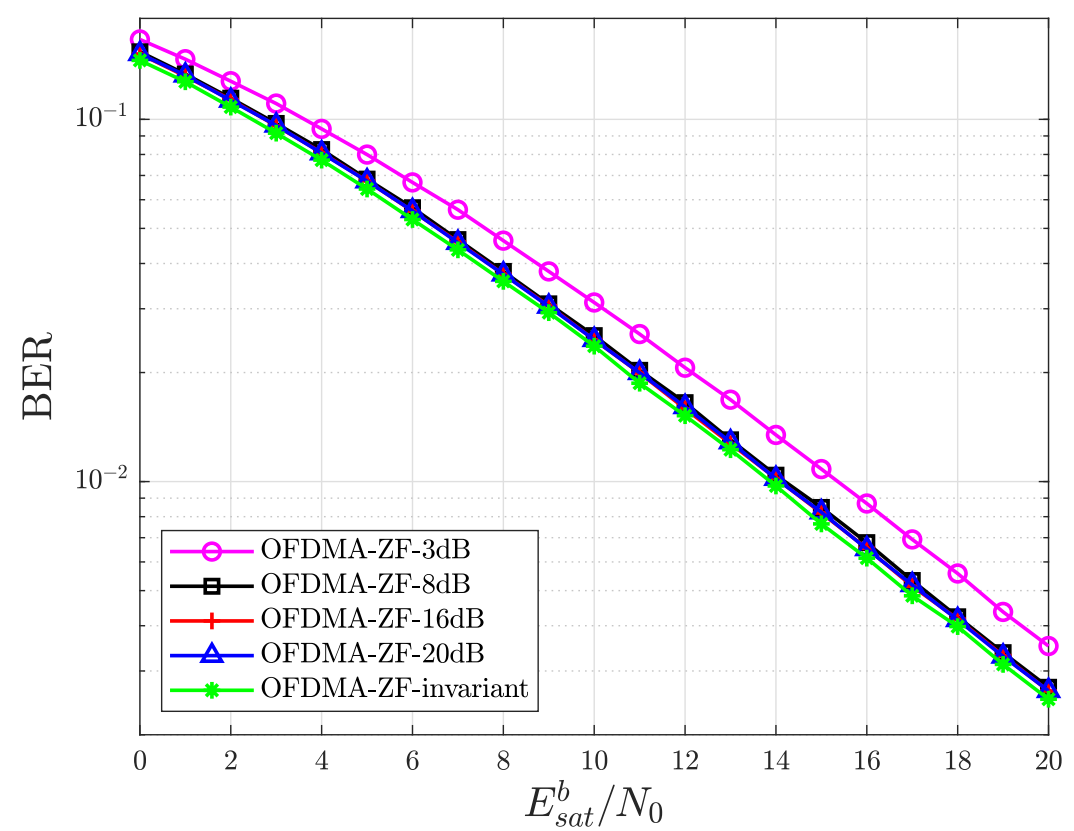

Figure 4.3: BER vs $E_{\text {sat }}^{b} / N_{0}$ curves to OFDMA non-linear systems without considering the OBO with $L=4, N=8, K=8, B=8$ and $P=4$, in downlink configuration and using the ZF equalizer.

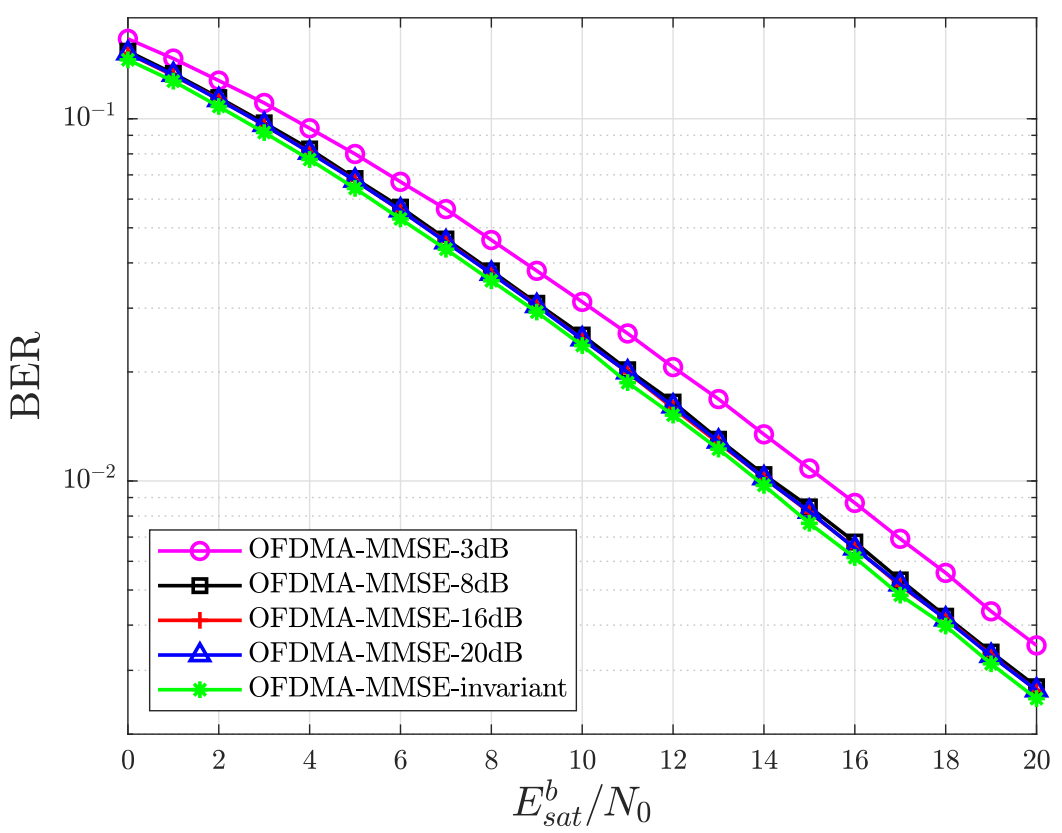

Figure 4.4: BER vs $E_{\text {sat }}^{b} / N_{0}$ curves to OFDMA non-linear systems without considering the $\mathrm{OBO}$ with $L=4, N=8, K=8, B=8$ and $P=4$, in downlink configuration and using the MMSE equalizer. 
time-invariant linear channels. The performance curves for CS-CDMA system are obtained using (4-37). When the OBO values are higher, the value of the

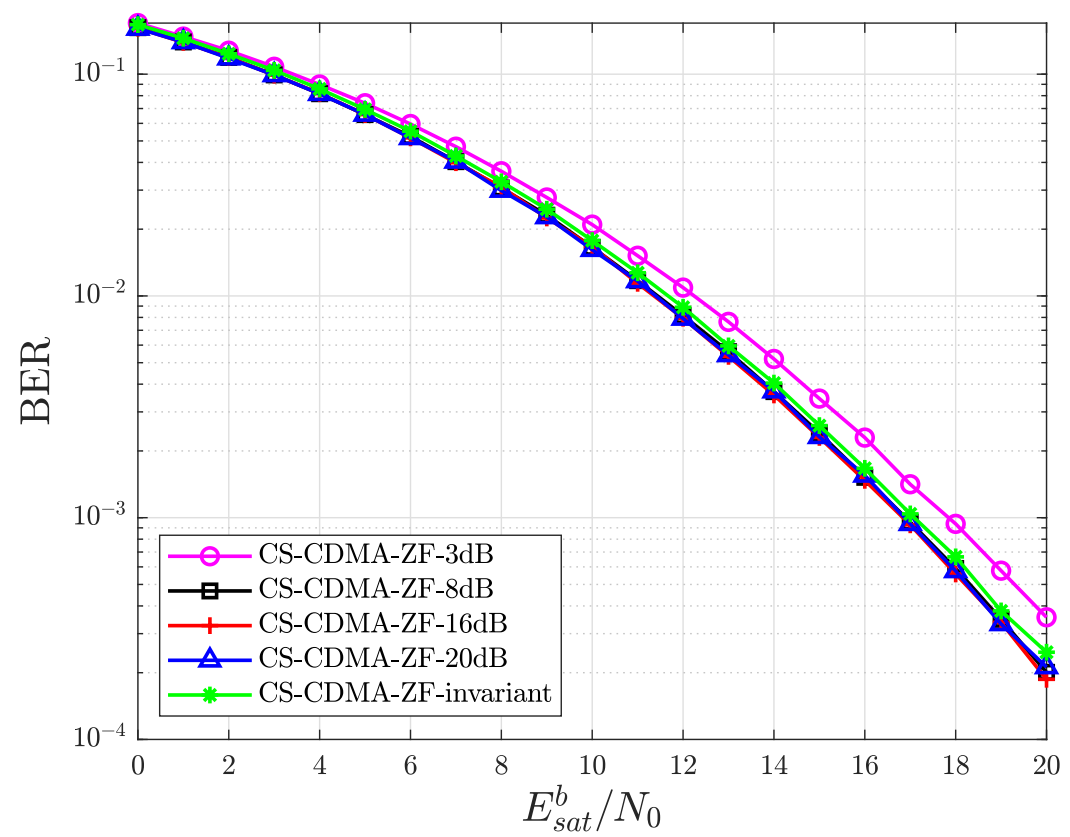

Figure 4.5: BER vs $E_{\text {sat }}^{b} / N_{0}$ curves to CS-CDMA non-linear systems without considering the $\mathrm{OBO}$ with $L=4, N=8, K=8, B=8$ and $P=4$, in downlink configuration and using the $\mathrm{ZF}$ equalizer.

adjust parameter $\alpha$ is equal to $\sqrt{E_{\text {out }}}$, and $\mathbf{z}=\mathbf{w}$. In this case, the system becomes a linear and time-invariant system as studied in Chapter 2.

Figures 4.7 and 4.8 illustrate the performance curves of the OFDMA system, considering the OBO as expressed in (4-22) and using the ZF and MMSE equalizer respectively. Both figures show the curves with the same performance since the channel matrix is diagonal. With the OBO effect, the curves with higher OBO suffer a greater drop in performance since the OBO introduces an amplification in the curves. This behavior is evidenced in (4-22).

In CS-CDMA, the performance curves were also analyzed considering the effects induced by the OBO. Figures 4.9 and 4.10 illustrate the results of a CS-CDMA system in a time-invariant non-linear channel using (4-37). Both figures show the same behavior previously observed for the OFDMA curves illustrated in figures 4.7 and 4.8. We can notice that in CS-CDMA, the results with the ZF equalizer were very close to the MMSE results. Both results are illustrated in figures 4.9 and 4.10 .

After analyzing both techniques, the results obtained in this section evidence that CS-CDMA achieves better performance results than the OFDMA system. In this section, we explored the behavior of the two systems by intro- 


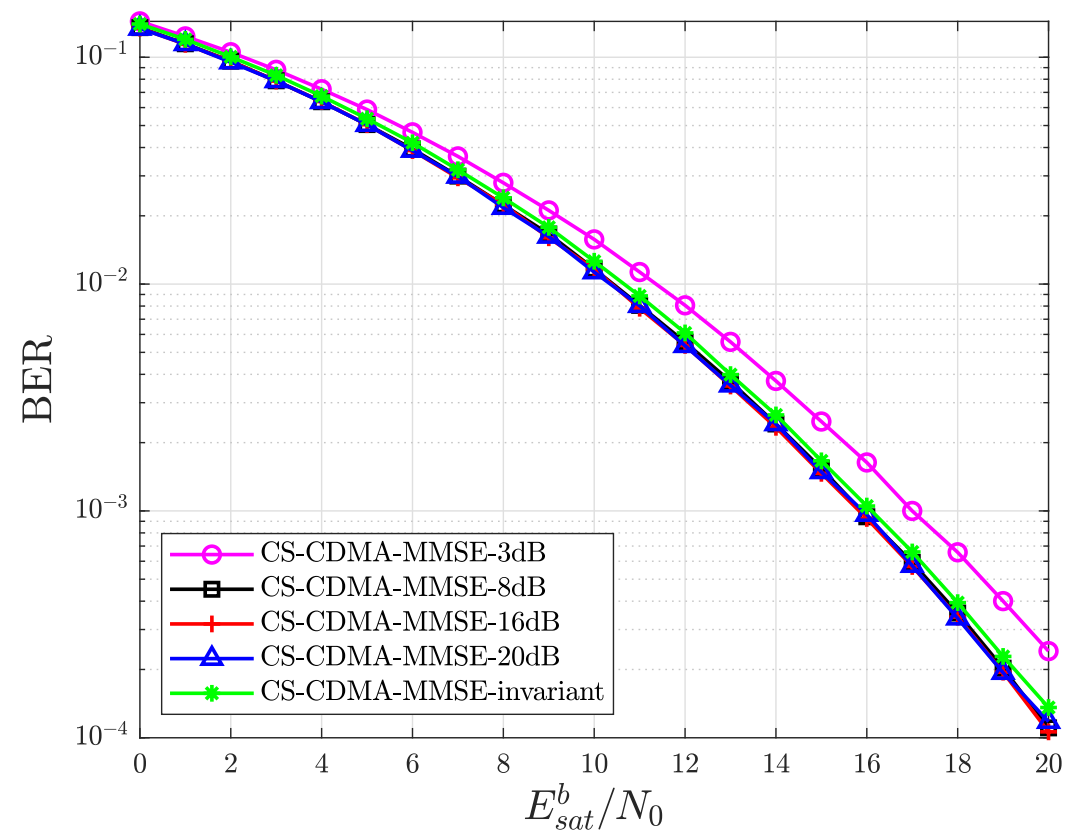

Figure 4.6: BER vs $E_{\text {sat }}^{b} / N_{0}$ curves to CS-CDMA non-linear systems without consider the OBO with $L=4, N=8, K=8, B=8$ and $P=4$, in downlink configuration and using the MMSE equalizer.

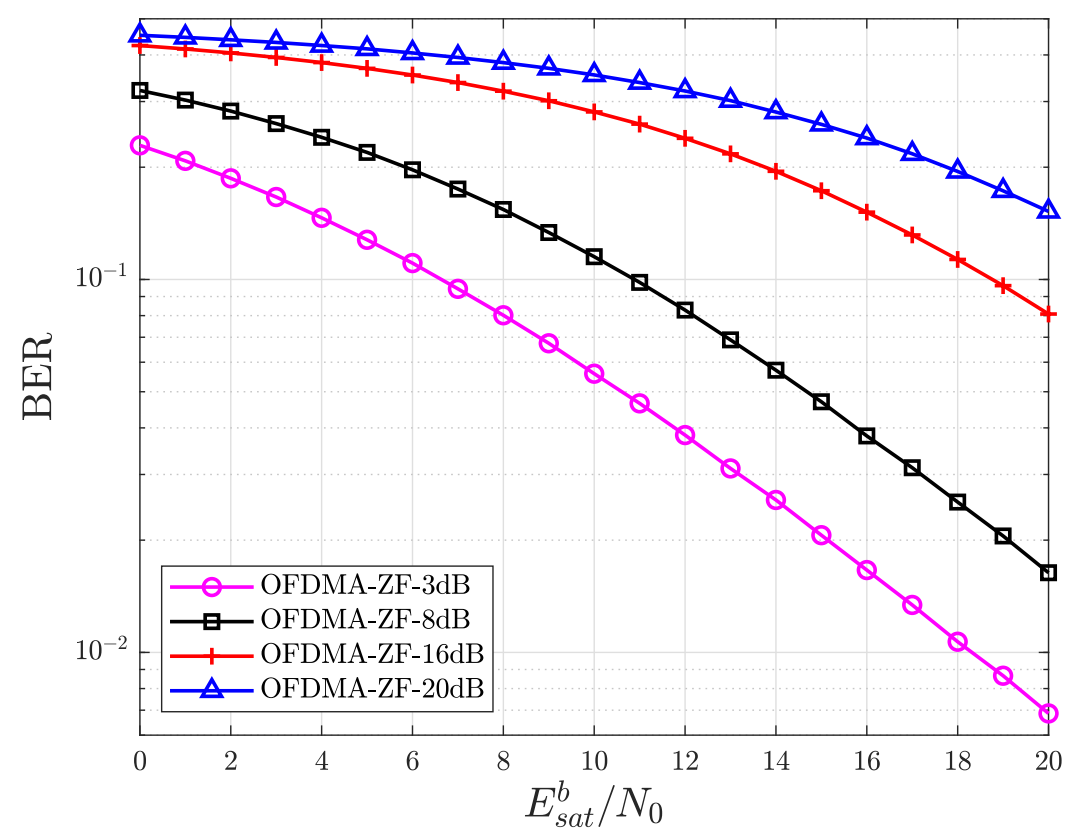

Figure 4.7: BER vs $E_{\text {sat }}^{b} / N_{0}$ curves to OFDMA non-linear systems considering the OBO with $L=4, N=8, K=8, B=8$ and $P=4$, in downlink configuration and using the $\mathrm{ZF}$ equalizer. 


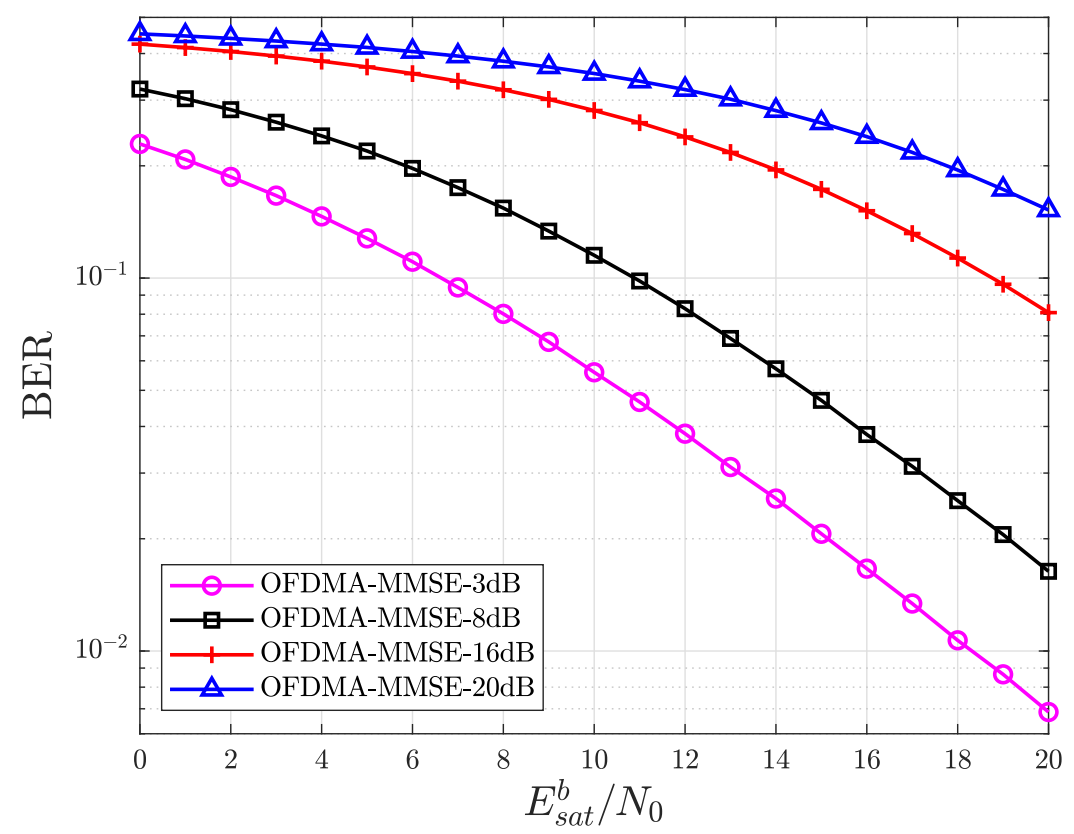

Figure 4.8: BER vs $E_{\text {sat }}^{b} / N_{0}$ curves to OFDMA non-linear systems considering the OBO with $L=4, N=8, K=8, B=8$ and $P=4$, in downlink configuration and using the MMSE equalizer.

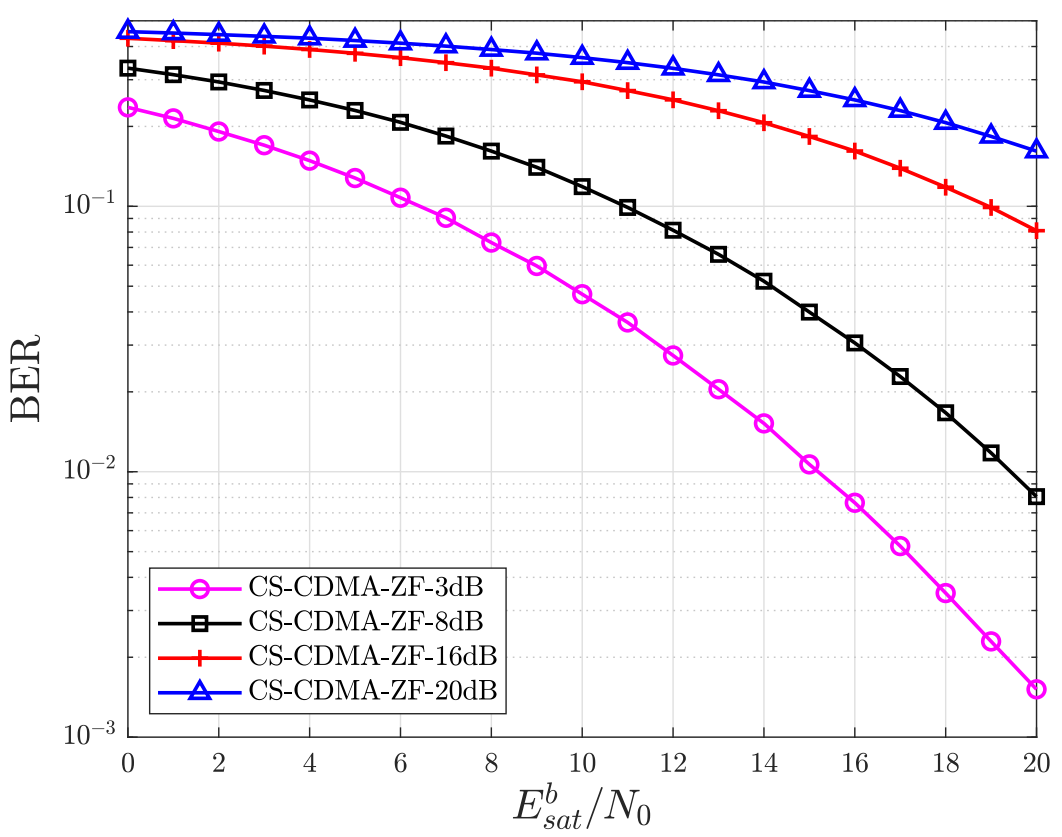

Figure 4.9: BER vs $E_{\text {sat }}^{b} / N_{0}$ curves to CS-CDMA non-linear systems considering the $\mathrm{OBO}$ with $L=4, N=8, K=8, B=8$ and $P=4$, in downlink configuration and using the $\mathrm{ZF}$ equalizer. 


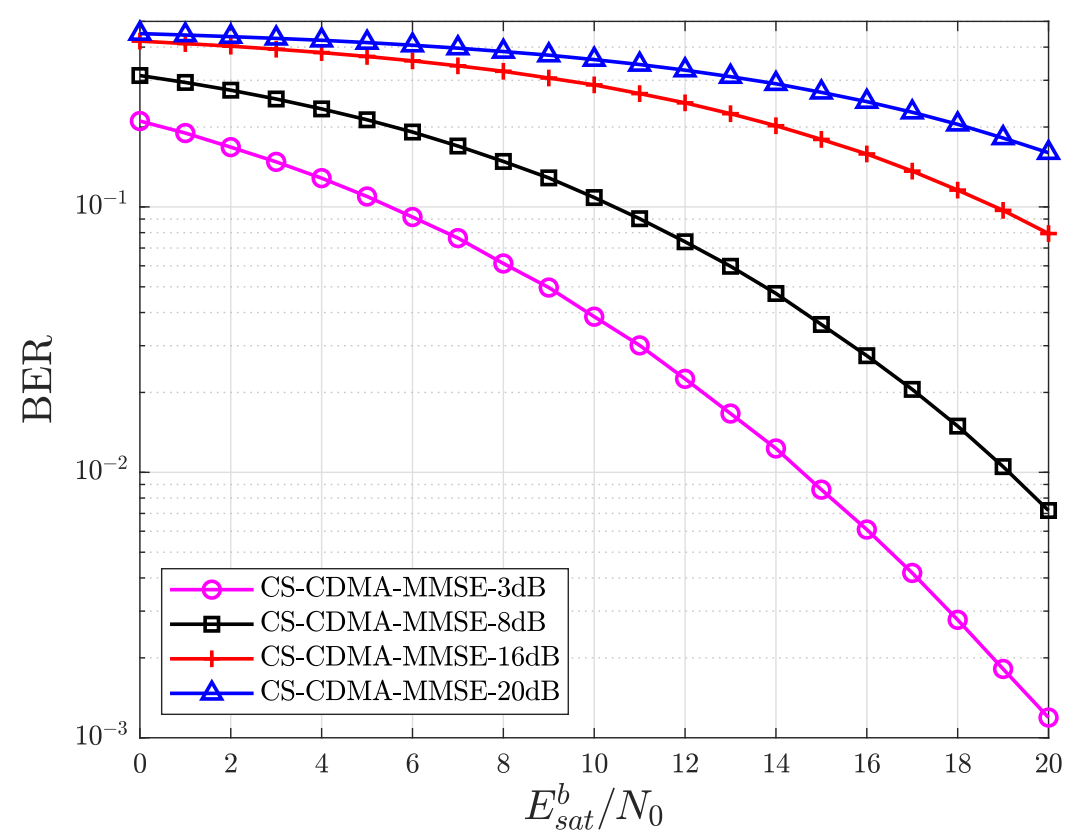

Figure 4.10: BER vs $E_{\text {sat }}^{b} / N_{0}$ curves to CS-CDMA non-linear systems considering the OBO with $L=4, N=8, K=8, B=8$ and $P=4$, in downlink configuration and using the MMSE equalizer.

ducing a non-linearity and it was evidenced that both systems were similarly affected. 


\section{5}

\section{Conclusions}

This dissertation focused on the analysis of two orthogonal multiple access techniques: OFDMA and CS-CDMA in different types of propagation channels. These techniques allow taking advantage of the channel's resources to increase the number of active users that the systems support. Matrix models were proposed for both techniques in each of the channel types studied.

In Chapter 2, the behavior of OFDMA and CS-CDMA systems in timeinvariant linear channels was analyzed. The matrix models for both orthogonal multiple access methods were presented. A new OFDMA matrix model approach was proposed for the uplink and downlink configuration. For CSCDMA systems, the matrix model using ZP as guard interval was suggested, where equalization in frequency domain was not necessary. Numerical results were also presented using the linear detection techniques ZF and MMSE. The performance results indicate a significant superiority of the CS-CDMA system. A comparison between the use of ZP and CP guard intervals in CS-CDMA systems was also included in this study, where the factors that led to ZP obtaining better results were observed.

A spectral analysis was also presented in Chapter 2, where expressions to compute the PSD and the out-of-band emission for both system were obtained. With the analysis carried out in this part, we demonstrate that for the same information symbol rate, the spectrum of the CS-CDMA system is wider than the OFDMA, which means that in this aspect the OFDMA system has better results.

In Chapter 3, a study of both systems in time-varying linear channels was carried out. Slow and fast variations were studied in this chapter for OFDMA and CS-CDMA systems. Matrix models for both multiple access systems were proposed using $\mathrm{CP}$ and ZP respectively. The channel model used by both systems was explained (Clarke model). The linear detectors ZF and MMSE were used to obtain the performance curves of each system in time-varying linear channels. The numerical results evidenced that OFDMA systems were more robust against the fast variations introduced, but could not overcome the results of the CS-CDMA performance curves.

The time-invariant non-linear channel were discussed in Chapter 4. We 
propose a model that takes into account the non-linearity introduced in the channel. Mathematical expressions that characterize both systems were obtained in this type of channels. At the end of the chapter, numerical results were illustrated, where the influence of the $\mathrm{OBO}$ and IBO was also analyzed. The performance curves have shown that the CS-CDMA system achieved better performance although both methods were similarly affected by non-linearities introduced by the overall communications channel.

In general terms the CS-CDMA system obtained better BER performance despite OFDMA achieving better results in terms of spectral occupation. As future works, we suggest the extension of the analysis by considering comparisons of complexity between both methods. 


\section{Bibliography}

[1] DAVID, R. P.; SAMPAIO-NETO, R. Técnica de Estimação de Canal utilizando Símbolos Piloto em Sistemas OFDM. Master dissertation, Pontifícia Universidade Católica do Rio de Janeiro, Rio de Janeiro, 2007.

[2] AREVAlO, L.; A. MEDinA, C.; SAMPAIO-NETO, R. Playing with Blocks: A New Combination of Block Transmission and the CDMA Multiple Access Technique. IEEE 81st Vehicular Technology Conference (VTC Spring), p. 1-4, 2015.

[3] MILADINOVIC, I.; SCHEFER-WENZL, S. NFV enabled IoT architecture for an operating room environment. In: 2018 IEEE 4th World Forum on Internet of Things (WF-loT), p. 98-102, 2018.

[4] LE, T., N. VAN, D.; RYU, E. Real-time 360-degree video streaming over millimeter wave communication. In: 2018 International Conference on Information Networking (ICOIN), p. 857-862, 2018.

[5] K. E., P.; V., M.; V., N. Design and Simulation of OFDMA Transceiver for High Speed 5G Wireless Network using Immense PSO-GA. In: 2019 International Conference on Communication and Signal Processing (ICCSP), p. 975-979, 2019.

[6] CHOUDHURY, D. 5G wireless and millimeter wave technology evolution: An overview. In: 2015 IEEE MTT-S International Microwave Symposium, p. 1-4, 2015.

[7] MUQUET, B.; WANG, Z.; GIANNAKIS, G. B.; COURVILLE, M.; DUHAMEL, P. Cyclic Prefixing or Zero Padding for Wireless Multicarrier Transmissions? IEEE Transactions on Communications, 50(12):21362148, 2002.

[8] FAZEL, K.; KAISER, S. Multi-Carrier and Spread Spectrum Systems: From OFDM and MC-CDMA to LTE and WiMAX. Wiley, 2008.

[9] CHOULY, A.; BRAJAL, A.; JOURDAN, S. Orthogonal multicarrier techniques applied to direct sequence spread spectrum 
CDMA systems. In: PROCEEDINGS OF GLOBECOM '93. IEEE GLOBAL TELECOMMUNICATIONS CONFERENCE, p. 1723-1728, 1993.

[10] DASILVA, V.; SOUSA, E. S. Performance of orthogonal CDMA codes for quasi-synchronous communication systems. In: PROCEEDINGS OF 2ND IEEE INTERNATIONAL CONFERENCE ON UNIVERSAL PERSONAL COMMUNICATIONS, p. 995-999, 1993.

[11] YANG, L. L.; HANZO, L. Multicarrier DS-CDMA: a multiple access scheme for ubiquitous broadband wireless communications. 41(10):116-124, 2003.

[12] MADHUKUMAR, A.; CHIN, F.; LIANG, Y.; YANG, K. Single Carrier Cyclic Prefix-Assisted CDMA System with Frequency Domain Equalization for High Data Rate Transmission. In: EURASIP J. WIRELESS COMMUNICATION NETWORK, p. 149-160, 2004.

[13] BAUM, K. L.; THOMAS, T. A.; VOOK, F. W.; NANGIA, V. Cyclic-prefix CDMA: an improved transmission method for broadband DSCDMA cellular systems. In: IEEE WIRELESS COMMUNICATIONS AND NETWORKING CONFERENCE RECORD, p. 183-188, 2002.

[14] LI, J.; WU, X.; LAROIA, R. OFDMA Mobile Broadband Communications: A Systems Approach. Cambridge University Press, 2013.

[15] CHANG, R.; GIBBY, R. A Theoretical Study of Performance of an Orthogonal Multiplexing Data Transmission Scheme. IEEE Transactions on Communication Technology, 16(4):529-540, 1968.

[16] VAN NEE, R.; PRASAD, R. OFDM for Wireless Multimedia Communications. Artech House, Inc., USA, 1st edition, 2000.

[17] CARVALHO, L. O.; SAMPAIO-NETO, R. Detecção de sinais em sistemas OFDM operando em canais que variam rapidamente no tempo. Master dissetation, Pontifícia Universidade Católica do Rio de Janeiro, Rio de Janeiro, 2019.

[18] BACKX, F. D.; SAMPAIO-NETO, R. Estimação de canal e detecção cega de sinais em sistemas de transmissão OFDM. Phd thesis, Pontifícia Universidade Católica do Rio de Janeiro, Rio de Janeiro, 2009.

[19] HAMPTON, J. R. Introduction to MIMO Communications. Cambridge University Press, USA, 1st edition, 2014. 
[20] FUKUSA, R. M.; ABRÃO, T. Sistema de Comunicação OFDM: da Fundamentação Conceitual à Implementação em Plataforma DSP. Trabalho de conclusão de curso, Universidade Estadual de Londrina, Londrina, 2016.

[21] PEREIRA, D.; SAMPAIO-NETO, R.; DAVID, R. P. Transmissão ChipSpread CDMA em Condições não Ideais: Uma Análise Comparativa. Master dissertation, Pontifícia Universidade Católica do Rio de Janeiro, Rio de Janeiro, 2016.

[22] QIYUE, Y.; WEIXIAO, M.; FUMIYUKI, A. A code assignment algorithm for multi-user/multi-rate 2-dimensional block spread SCCDMA. In: 6TH INTERNATIONAL ICST CONFERENCE ON COMMUNICATIONS AND NETWORKING IN CHINA (CHINACOM), p. 156-160, 2011.

[23] SKLAR, B. Rayleigh fading channels in mobile digital communication systems .I. Characterization. 6th International ICST Conference on Communications and Networking in China (CHINACOM), 35(7):90-100, 1997.

[24] PEREIRA, D.; ARÉVAlOS, L.; DAVID, R. P.; SAMPAIO-NETO, R. ChipSpread CDMA Transmission: A Comparative Analysis. In: XXXIV Simpósio Brasileiro de Telecomunicações e Processamento de Sinais (SBrT 2016), p. 428-432, 2016.

[25] VISWANATHAN, M. Simulation of Digital Communication systems using Matlab. Amazon Kindle, 2nd edition, 2013.

[26] RODRIGUES, M. R. D. Modelling and Performance Assessment of OFDM Communication Systems in the Presence of Nonlinearities. Phd thesis, University College London, England, 2002.

[27] HILARIO-TACURI, A.; P. FORTES, J. M.; SAMPAIO-NETO, R. Analytical Spectral Evaluation of GFDM Systems Over Non-Linear Channels with Memory. In: 2018 IEEE 10th Latin-American Conference on Communications (LATINCOM), p. 1-5, 2018.

[28] BENEDETTO, S.; BIGLIERI, E.; CASTELLANI, V. Digital transmission theory. Prentice Hall, USA, 1987.

[29] CUADRADO, D. N.; SAMPAIO-NETO, R.; CAL-BRAZ, J. Antenna Selection in MIMO-OFDM systems. Master dissertation, Pontifícia Universidade Católica do Rio de Janeiro, Rio de Janeiro, 2020. 
A

\section{PSD of Block Transmission Systems}

Let $x(t)$ for $t \in \mathbb{R}$, a stochastic process (SP) with Power Spectral Density (PSD) given by

$$
\mathbf{S}_{x}(f)=\lim _{T_{0} \rightarrow \infty} \frac{1}{T_{0}} \mathbb{E}\left[\left|\int_{-\frac{T_{0}}{2}}^{\frac{T_{0}}{2}} x(t) e^{-j 2 \pi f t} d t\right|^{2}\right],
$$

where $\int_{-\frac{T_{0}}{2}}^{\frac{T_{0}}{2}} x(t) e^{-j 2 \pi f t} d t$ is the Fourier Transform of $x(t)$ restricted to interval $\left[-\frac{T_{0}}{2} ;-\frac{T_{0}}{2}\right]$.

Considering the $D \times 1$ vector $\chi^{(i)}$ defined by multiplication of the transmission matrix $\boldsymbol{\Psi}$ with dimension $D \times Z$ and the $Z \times 1$ vector $\mathbf{d}_{i}$ that contains information symbols, we have

$$
\chi^{(i)}=\left[\begin{array}{c}
\chi^{(i)}(0) \\
\chi^{(i)}(1) \\
\vdots \\
\chi^{(i)}(D-1)
\end{array}\right]=\boldsymbol{\Psi} \mathbf{d}_{i}
$$

It is assumed that the vectors

$$
\mathbf{d}_{i}=\left[\begin{array}{c}
s_{i 1} \\
s_{i 2} \\
\vdots \\
s_{i Z}
\end{array}\right], \quad i=0,1,2 \ldots
$$

are statistically independent with $\mathbb{E}\left[\mathbf{d}_{i}\right]=\mathbf{0}$ and $\mathbb{E}\left[\mathbf{d}_{i} \mathbf{d}_{i}^{H}\right]=\sigma_{s}^{2} \mathbf{I}$.

Let $x_{i}(t)$, the $i$-th transmitted symbol block, such that

$$
x_{i}(t)=\sum_{l=0}^{D-1} \chi^{(i)}(l) g\left(t-l T_{s}\right),
$$

where $T_{s}=1 / R_{s}, R_{s}$ is the transmitted symbol rate and $g(t)$ represents the transmission pulse.

The signal $x(t)$ is the result of the sequential transmission of blocks $x_{i}(t)$ and can be expressed as

$$
x(t)=\sum_{i=\infty}^{\infty} x_{i}(t-i T)
$$


where $T=D T_{s}$.

The expression (A-1) can be computed by initially restricting (A-5) to a finite number of terms, such that

$$
\mathbf{S}_{x}(f)=\lim _{T^{\prime} \rightarrow \infty} \frac{1}{T^{\prime}} \mathbb{E}\left[\left|\mathcal{F}\left[\sum_{i=-K}^{+K} x_{i}(t-i T)\right]\right|^{2}\right]
$$

where the operator $\mathcal{F}\left[\right.$.] computes the Fourier transform and $T^{\prime}=(2 K+1) T=$ $(2 K+1) D T_{s}$. The expression (A-6) after Fourier transform computation is expressed as

$$
\mathbf{S}_{x}(f)=\lim _{K \rightarrow \infty} \frac{1}{(2 K+1) T} \mathbb{E}\left[\left|\sum_{i=-K}^{+K} X_{i}(f) e^{-j 2 \pi i f T}\right|^{2}\right]
$$

where

$$
\begin{aligned}
X_{i}(f) & =\mathcal{F}\left[x_{i}(t)\right] \\
& =\mathcal{F}\left[\sum_{l=0}^{D-1} \chi^{(i)}(l) g(t-l T s)\right] \\
& =\sum_{l=0}^{D-1} \chi^{(i)}(l) G(f) e^{-j 2 \pi i f T_{s}},
\end{aligned}
$$

and $G(f)$ denotes the spectrum of transmission pulse.

Expanding the expected value in (A-7) results

$$
\mathbf{S}_{x}(f)=\lim _{K \rightarrow \infty} \frac{1}{(2 K+1) T} \sum_{i=-K}^{+K} \sum_{m=-K}^{+K} \mathbb{E}\left[X_{i}(f) X_{m}^{*}(f)\right] e^{-j 2 \pi(i-m) f T},
$$

and using (A-8) the expected value in (A-9) is expressed as

$$
\mathbb{E}\left[X_{i}(f) X_{m}^{*}(f)\right]=|G(f)|^{2} \sum_{l=0}^{D-1} \sum_{n=0}^{D-1} \mathbb{E}\left[\chi^{(i)}(l) \chi^{(m) *}(n)\right] e^{-j 2 \pi(l-n) f T_{s}} .
$$

The vectors $\boldsymbol{\chi}_{(i)}$ are considered statistically independent with $\mathbf{E}\left[\boldsymbol{\chi}^{(i)}\right]=0$, such that

$$
\mathbb{E}\left[\chi^{(i)}(l) \chi^{(m) *}(n)\right]= \begin{cases}0 & , i \neq m \\ R_{x}(l, n) l, n=0,1, \ldots, D-1 & , i=m .\end{cases}
$$

Using (A-10) and (A-11), we have that

$$
\mathbb{E}\left[X_{i}(f) X_{m}^{*}(f)\right]= \begin{cases}0 & , i \neq m \\ |G(f)|^{2} \sum_{l=0}^{D-1} \sum_{n=0}^{D-1} R_{x}(l, n) e^{-j 2 \pi(l-n) f T_{s}} & , i=m .\end{cases}
$$

Substituting (A-12) in (A-9) and taking into account that $\mathbb{E}\left[\left|X_{i}(f)\right|^{2}\right]$ does not 
depend of the $i$ index, we arrive at

$$
\begin{aligned}
\mathbf{S}_{x}(f) & =\lim _{K \rightarrow \infty} \frac{1}{(2 K+1) T} \sum_{i=-K}^{+K} \mathbb{E}\left[\left|X_{i}(f)\right|^{2}\right] \\
& =\lim _{K \rightarrow \infty} \frac{1}{(2 K+1) T} \sum_{i=-K}^{+K} \mathbb{E}\left[\left|X_{0}(f)\right|^{2}\right](2 K+1) \\
& =\frac{1}{T}|G(f)|^{2} \sum_{l=0}^{D-1} \sum_{n=0}^{D-1} R_{x}(l, n) e^{-j 2 \pi(l-n) f T_{s}},
\end{aligned}
$$

where $R_{x}(l, n)$ with $l$ and $n \in 0,1, \ldots, D-1$, are the component of the autocorrelation matrix of the transmitted blocks

$$
\begin{aligned}
\mathbf{R}_{x} & =\mathbb{E}\left[\chi^{(i)} \chi^{(i) H}\right] \\
& =\mathbb{E}\left[\boldsymbol{\Psi}_{\left.\mathbf{s}_{i} \mathbf{s}_{i}^{H} \boldsymbol{\Psi}^{H}\right]}\right. \\
& =\boldsymbol{\Psi} \mathbb{E}\left[\mathbf{s}_{i} \mathbf{s}_{i}^{H}\right] \boldsymbol{\Psi}^{H} \\
& =\boldsymbol{\Psi} \sigma_{s}^{2} \mathbf{I} \boldsymbol{\Psi}^{H} \\
& =\sigma_{s}^{2} \boldsymbol{\Psi} \boldsymbol{\Psi}^{H}
\end{aligned}
$$

Using $T=D T_{s}$, the expression (A-13) can be rewritten as

$$
\mathbf{S}_{x}(f)=\frac{1}{T_{s}}|G(f)|^{2} \frac{1}{D} \sum_{l=0}^{D-1} \sum_{n=0}^{D-1} R_{x}(l, n) e^{-j 2 \pi(l-n) f T_{s}} .
$$




\section{B}

\section{Analytical Expression of BER for OFDM Systems}

Let $\mathbf{r}$ be the processed received vector in linear time-invariant channel as expressed by (2-9) and (2-10), we omitted the time index $i$ for ease of the notation,

$$
\begin{aligned}
\mathbf{r} & =\left[\begin{array}{cccc}
q_{1} & 0 & \ldots & 0 \\
0 & q_{2} & \ddots & \vdots \\
\vdots & \ddots & \ddots & 0 \\
0 & \ldots & 0 & q_{N}
\end{array}\right]\left[\begin{array}{c}
d_{1} \\
d_{2} \\
\vdots \\
d_{N}
\end{array}\right]+\left[\begin{array}{c}
n_{1} \\
n_{2} \\
\vdots \\
n_{N}
\end{array}\right] \\
& =\left[\begin{array}{c}
q_{1} d_{1} \\
q_{2} d_{2} \\
\vdots \\
q_{N} d_{N}
\end{array}\right]+\left[\begin{array}{c}
n_{1} \\
n_{2} \\
\vdots \\
n_{N}
\end{array}\right] .
\end{aligned}
$$

Assuming, as in Section 2.3, that the channel vector $\mathbf{h}$ in (2-10) is complex Gaussian with $\mathbb{E}[\mathbf{h}]=\mathbf{0}, \mathbb{E}\|\mathbf{h}\|^{2}=1$ and statistically independent components, then $q_{i}$ with $i=1, \ldots, N$ are complex Gaussian variables with null mean and $\mathbb{E}\left[\left|q_{i}\right|^{2}\right]=1$. The components of data vector $\mathbf{d}$ are detected separately. The detection of the first component $\left(d_{1}\right)$ uses the observation

$$
r_{1}=q_{1} d_{1}+n_{1}
$$

Both ZF and MMSE equalization and detection can be performed by first multiplying $r_{1}$ by $q_{1}^{*}$, leading to

$$
\begin{aligned}
r_{1}^{\prime} & =\left|q_{1}\right|^{2} d_{1}+q_{1}^{*} n_{1} \\
& =\left|q_{1}\right|^{2} d_{1}+n_{1}^{\prime}
\end{aligned}
$$

where $n_{1}^{\prime}=q_{1}^{*} n_{1}$. Given $q_{1}, n_{1}^{\prime}$ is a complex random Gaussian variable with zero mean and

$$
\begin{aligned}
\mathbb{E}\left[\left|n_{1}^{\prime}\right|^{2}\right] & =\mathbb{E}\left[q_{1}^{*} n_{1} n_{1}^{*} q_{1}\right] \\
& =q_{1}^{*} \mathbb{E}\left[\left|n_{1}\right|^{2}\right] q_{1} \\
& =\left|q_{1}\right|^{2} \sigma^{2},
\end{aligned}
$$


where $\sigma^{2}=N_{0}$. Using (B-3), the signal-to-noise ratio given $q_{1}$ can be obtained from the expressions for signal and noise, such that

$$
\begin{aligned}
& \text { Signal: } \mathbb{E}\left[\left(\left|q_{1}\right|^{2}\left|d_{1}\right|\right)^{2}\right]=\left|q_{1}\right|^{4} \mathbb{E}\left[\left|d_{1}\right|^{2}\right]=\left|q_{1}\right|^{4} E_{s} \\
& \text { Noise: } \mathbb{E}\left[\left|n_{1}^{\prime}\right|^{2}\right]=\left|q_{1}\right|^{2} N_{0},
\end{aligned}
$$

where $E_{s}$ is the energy of a symbol of the data symbol vector. In this way, the signal-to-noise ratio given $q_{1}$ is expressed by

$$
\mathrm{SNR}=\frac{\text { Signal }}{\text { Noise }}=\left|q_{1}\right|^{2} \frac{E_{s}}{N_{0}} .
$$

With these results, assuming QPSK modulation the BER given $q_{1}$ is given by

$$
\begin{aligned}
B E R_{q_{1}} & =Q(\mathrm{SNR}) \\
& =Q\left(\sqrt{\left|q_{1}\right|^{2} \frac{E_{s}}{N_{0}}}\right) .
\end{aligned}
$$

The expected value can be applied in (B-8), such that

$$
B E R_{q_{1}}=\mathbb{E}\left[Q\left(\sqrt{\left|q_{1}\right|^{2} \frac{E_{s}}{N_{0}}}\right)\right]=\mathbb{E}\left[Q\left(\sqrt{x \frac{E_{s}}{N_{0}}}\right)\right],
$$

where $x=\left|q_{1}\right|^{2}$ is a random exponential variable with probability density function $p_{x}(x)=a e^{-a x} u(x)$ with $\mathbb{E}[x]=1$ and therefore, $a=1$. Applying these concepts, the BER can be expressed by

$$
\begin{aligned}
\mathrm{BER} & =\int_{0}^{\infty} Q\left(\sqrt{x \frac{E_{s}}{N_{0}}}\right) e^{-x} d x \\
& =\frac{1}{2}\left[1-\sqrt{\frac{E_{s} / N_{0}}{2+E_{s} / N_{0}}}\right] .
\end{aligned}
$$

Expression (B-10) evidences that for the considered random channel, the performance is independent of the number of taps and the power profile of the channel and of the number of sub-carriers of the OFDMA system. The BER can be computed using (B-10).

Figure B.1 shows the performance curves BER versus $E_{b} / N_{0}$ for 64 and 48 sub-carriers and a channel of 4 taps $(P=4)$, where $E_{b}$ is the energy per bit transmitted. The curves are coincident, which demonstrates that the performance of the OFDM system is independent of the number of sub-carriers. The analytical result illustrated in Figure B.1 is obtained using (B-10).

Figure B.2 illustrates the performance curves BER versus $E_{b} / N_{0}$ for 64 and 48 sub-carriers and a channel of 8 taps $(P=8)$. These results show that the OFDM technique is independent also of the number of taps of the 


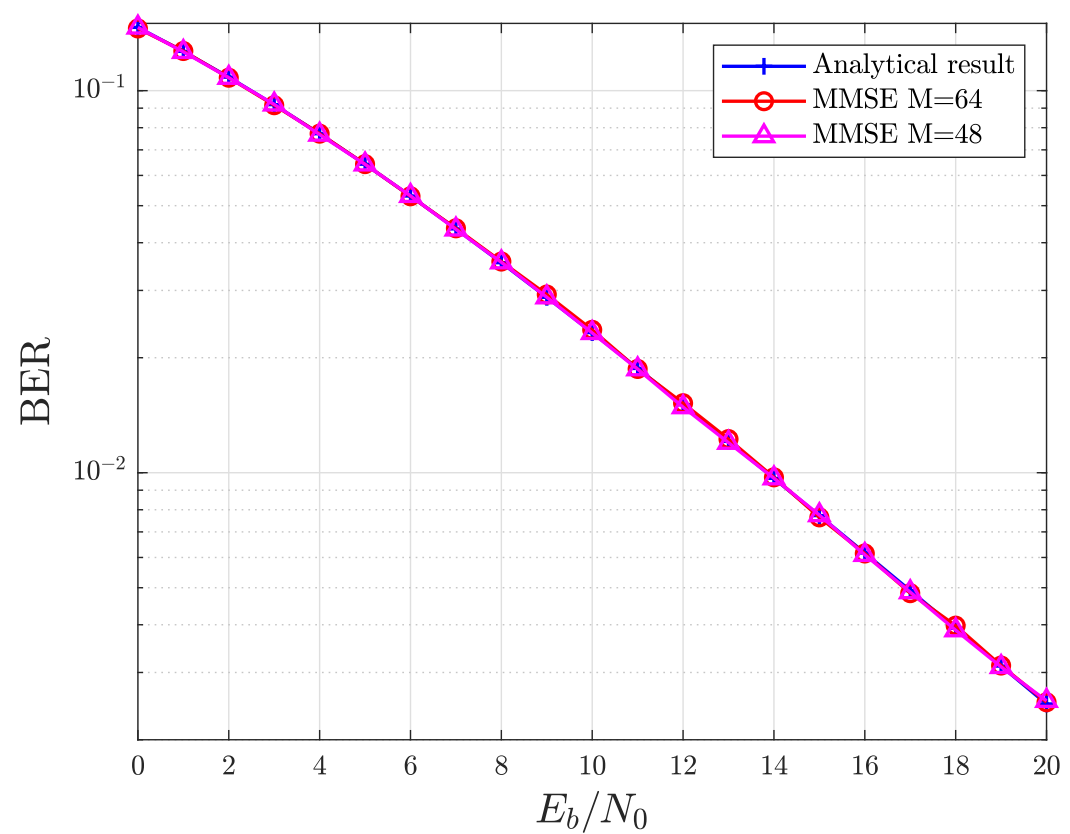

Figure B.1: BER vs $E_{b} / N_{0}$ curves of OFDM computed analytically and through simulation with $M=64$ and $48, L=4$ and $P=4$.

channels since the same analytical result obtained using (B-10) coincides with performance curves of two systems with MMSE detection of 64 and 48 subcarriers, respectively.

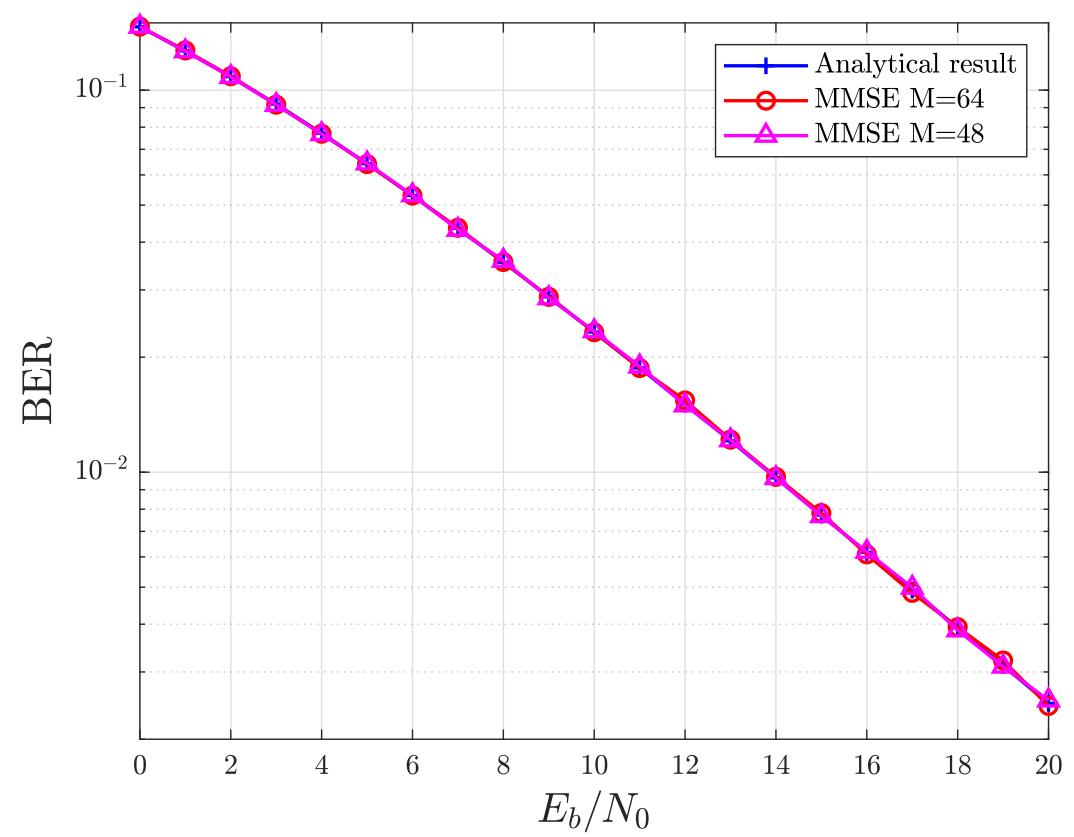

Figure B.2: BER vs $E_{b} / N_{0}$ curves of OFDM computed analytically and through simulation with $M=64$ and $48, L=8$ and $P=8$. 